\title{
Impact of Multi-Component Diffusion in Turbulent Combustion using Direct Numerical Simulations
}

Claudio Bruno* and Vaidyanathan Sankaran

United Technologies Research Center

411, Silver Lane, East Hartford, CT - 06108

Hemanth Kolla and Jacqueline $\mathrm{H}$. Chen

Sandia National Laboratories

Livermore, CA

*Corresponding Author. brunoc@utrc.utc.com

Dr. Claudio Bruno,

UTRC

411 Silver Lane, MS 129-29

East Hartford CT 06118-1127

USA 


\begin{abstract}
$\underline{\text { Abstract }}$
This paper presents the results of DNS of a partially premixed turbulent syngas/air flame at atmospheric pressure. The objective was to assess the importance and possible effects of molecular transport on flame behavior and structure. To this purpose DNS were performed at with two proprietary DNS codes and with three different molecular diffusion transport models: fully multi-component, mixture averaged, and imposing the Lewis number of all species to be unity.

Results indicate that

1. At the Reynolds numbers of the simulations $\left(\operatorname{Re}_{\text {turb }}=600, \operatorname{Re}=8000\right)$ choice of molecular diffusion models affects significantly the temperature and concentration fields;

2. Assuming Le $=1$ for all species predicts temperatures up to $250 \mathrm{~K}$ higher than the physically realistic multi-component model;

3. Faster molecular transport of lighter species changes the local concentration field and affects reaction pathways and chemical kinetics.

A possible explanation for these observations is provided in terms of species diffusion velocity that is a strong function of gradients: thus, at sufficiently large Reynolds numbers, gradients and their effects tend to be large. The preliminary conclusion from these simulations seems to indicate molecular diffusion as the third important mechanism active in flames besides convective transport and kinetics. If confirmed by further DNS and measurements, molecular transport in high intensity turbulent flames will have to be realistically modeled to accurately predict emissions (gaseous and particulates) and other combustor performance metrics.
\end{abstract}




\section{Introduction}

Commonly thought unimportant, molecular diffusion effects in turbulent flames are attracting the attention of combustion researchers. In fact, molecular transport is driven by the same collisional physics as chemical kinetics and is the last step preceding reaction, suggesting that at the scale where kinetics takes place diffusion should also have some significant role.

Measurements and simulations of turbulent flames indicate that diffusion of light species (e.g., $\mathrm{H}_{2}, \mathrm{H}, \mathrm{OH}$ and $\mathrm{H}_{2} \mathrm{O}$ ) may significantly influence species distribution and thus local temperature and emissions. That preferential (faster) diffusion of lighter species may be responsible for locally faster flame propagation was realized by Zel"dovich in his ,active centers" theory [1]. Measurements in premixed $\mathrm{H} 2 /$ air and stratified $\mathrm{CH}$ 4/air flames showing effects of diffusion have been reported [2] [3] [4] [5] [6] [7] [8] [9]. In jet- and coaxial burner flames these effects have been reported to decrease with the distance from the fuel injector and with increasing Reynolds number. However, that molecular diffusion may be important in turbulent combustion is the conclusion in reviews of this effect [8]; Direct Numerical Simulations (DNS) and Large Eddy Simulations (LES) results cited in [10] by [11] [12] [13] [14] [15] [16] suggest molecular diffusion is significant even in flames at Reynolds numbers $(\mathrm{Re})$ of order $10^{4}$.

Although many of the references cited infer or suggest preferential diffusion is present in turbulent flames, measurements in [6] [7] [17] and [18] were the first to purposely address this issue. They included species concentrations, equivalence ratio $(\phi)$ and carbon to hydrogen $(\mathrm{C} / \mathrm{H})$ ratio in a vertical laminar $\mathrm{CH} 4$ /air flame, plotted vs. local temperature, and measurements of the same quantities in a turbulent bluff-body burner up to Re $\sim 10000$. In both flames measurements were compared to predictions obtained with detailed kinetics using the zero dimensional CHEMKIN code and for the same flame in the laminar regime. (Since CHEMKIN cannot predict spatial profiles, results were reported as a function of temperature instead of, radial or axial distance across the burner.) Just as the laminar flame results were in agreement with CHEMKIN and showed the known effect of preferential diffusion [19] to manifest itself in locally non conserved $\mathrm{H}$ atoms and $\mathrm{C} / \mathrm{H}$ ratios, a similar effect was observed in the turbulent measurements; however, agreement with CHEMKIN was poor. Accordingly, the authors concluded that significant preferential diffusion was present also in the turbulent flame. Contributing to this conclusion was the observed enrichment of $\mathrm{CO}_{2}$ in the bluff-body recirculation region, plausibly explained by the preferential e.g., faster, emigration of lighter species such as $\mathrm{H}_{2} \mathrm{O}, \mathrm{H}_{2}$ and $\mathrm{H}$ away from regions of high concentration, therefore modifying local $\phi$ and $\mathrm{C} / \mathrm{H}$ ratio. The magnitude of this effect in turbulent flame was ascribed to sharper concentration gradients due to flame stretch which also increases with increasing Reynolds numbers. These measurements [7] and [17] also indicate that $\mathrm{H}_{2}$ concentration and local $\mathrm{H} / \mathrm{C}$ and equivalence ratios (ER) in lean and rich premixed methane/air atmospheric flames differ by up to $10-15 \%$ from those calculated with detailed kinetics. These results have been supported by LES calculations of equivalence ratio and $\mathrm{C} / \mathrm{H}$ ratio using mixture-averaged diffusion modeling [19]. A recent summary of questions on this issue, for both premixed and nonpremixed flames, is in [20]. 
These findings are intriguing because (i) they are observed in turbulent flames; (ii) effects are not negligible; (iii) they are observed in both experiments and DNS, and (iv) they are not only observed in hydrogen flames but also in hydrocarbon flames [8]. Molecular transport is only one of many extant questions about LES; however, the role of diffusion lends itself to be scrutinized more easily than, say, the two-way coupling between turbulence and chemical kinetics. In fact, exploring the impact of molecular diffusion matches current interest by industry and government organizations in improving the physical accuracy of LES; were the experimental results in [6] [7] [17] to be confirmed by DNS, it would help in convincing the combustion community of the importance of diffusion and of the need to account for it to enable predictive simulations. Moreover, the fact those spatial profiles measured in [6] [7] were compared only to CHEMKIN calculations were and still is a source of skepticism. This motivated the present authors to perform full DNS of a turbulent combustion test case focusing on the impact of molecular diffusion. Accordingly, the main goal of the present investigation is to quantitatively assess the impact of molecular diffusion in turbulent flames, at Reynolds numbers not too far from those of industrial interest and by means of DNS containing different diffusion models, with a linked goal to compare them and assess their performance.

\section{Molecular Diffusion}

The quantity characterizing molecular diffusion is the statistical diffusion velocity $\mathbf{V}_{i}$ of the $\mathrm{i}^{{ }^{\text {th }}}$ species. $\mathbf{V}_{i}$ appears in the ns species transport equations (1) present in the set of reactive Navier-Stokes equations (NSE), and obtained by integrating and statistically averaging the Boltzmann equation [21].

$$
\frac{D Y_{i}}{D t}=-\frac{1}{\rho} \nabla\left(\rho Y_{i} \boldsymbol{V}_{i}\right)+\frac{w_{i}}{\rho} \quad[\mathrm{i}=1,2, \ldots \mathrm{ns}]
$$

Neglecting approximations like Fick"s law, valid only in two-component mixtures, physically accurate $\mathbf{V}_{i}$ may be obtained either from kinetic theory, or derived from non-equilibrium thermodynamics. A convenient but less accurate diffusion model is that assuming each species "l" is characterized by a Lewis number Le $e_{i}$ constant in time and space. A brief description of $\mathbf{V}_{i}$ models is presented in Appendix A.

\section{Simulation Strategy}

To assess the impact of molecular diffusion in turbulent reacting flows, DNS were performed of a test case with the Mixture-Averaged Diffusion (MAD), Multi-Component Diffusion (MCD) and with constant Lewis number models and results were compared and analyzed. DNS eliminates the uncertainty due to turbulence closures and models invariably present in RANS and LES. However, computational burden of DNS significantly restricts the turbulent Reynolds number, $R e_{t}$, and the scale of the test case. Ideally, both should be close to conditions representative of real combustion applications. Such flows are characterized by intense shear, mixing between air, fuel and partial reaction products, and high temperatures/pressures; all of which may impact the magnitude of molecular diffusion and mixing. DNS also limits the kinetics complexity and therefore the type of fuel that can be economically simulated. In spite of these difficulties, DNS has been performed at a moderate turbulent Reynolds number using moderately complex chemical kinetics to highlight the impact of molecular diffusion models. 


\subsection{Test Case, Initial Conditions and Kinetics models}

The setup considered here is a temporally evolving jet/shear layer at atmospheric pressure (101325 Pa). The initial condition corresponds to a central rectangular syngas/air jet of height $\mathrm{H}$ $=2.56 \mathrm{~mm}$ with an equivalence ratio $\phi$ varying from 2 in the center to 1 in the outer region and flowing from left to right (see fig. 1b). This jet is sandwiched between two symmetrical rectangular jets formed by combustion products at $\phi \sim 1.0$ from a syngas/air mixture, thus creating a partially premixed flame. Note the burnt products are situated slightly outside the shear layer (separation distance $=5.12 \mathrm{~mm}=2 \mathrm{H}$ ). Inside the rich, unburnt central jet the equivalence ratio varies within the inner $1 \mathrm{H}$ region (see fig.2). Figure 1 shows also the contour map of the $\mathrm{O} 2$ mass fraction.

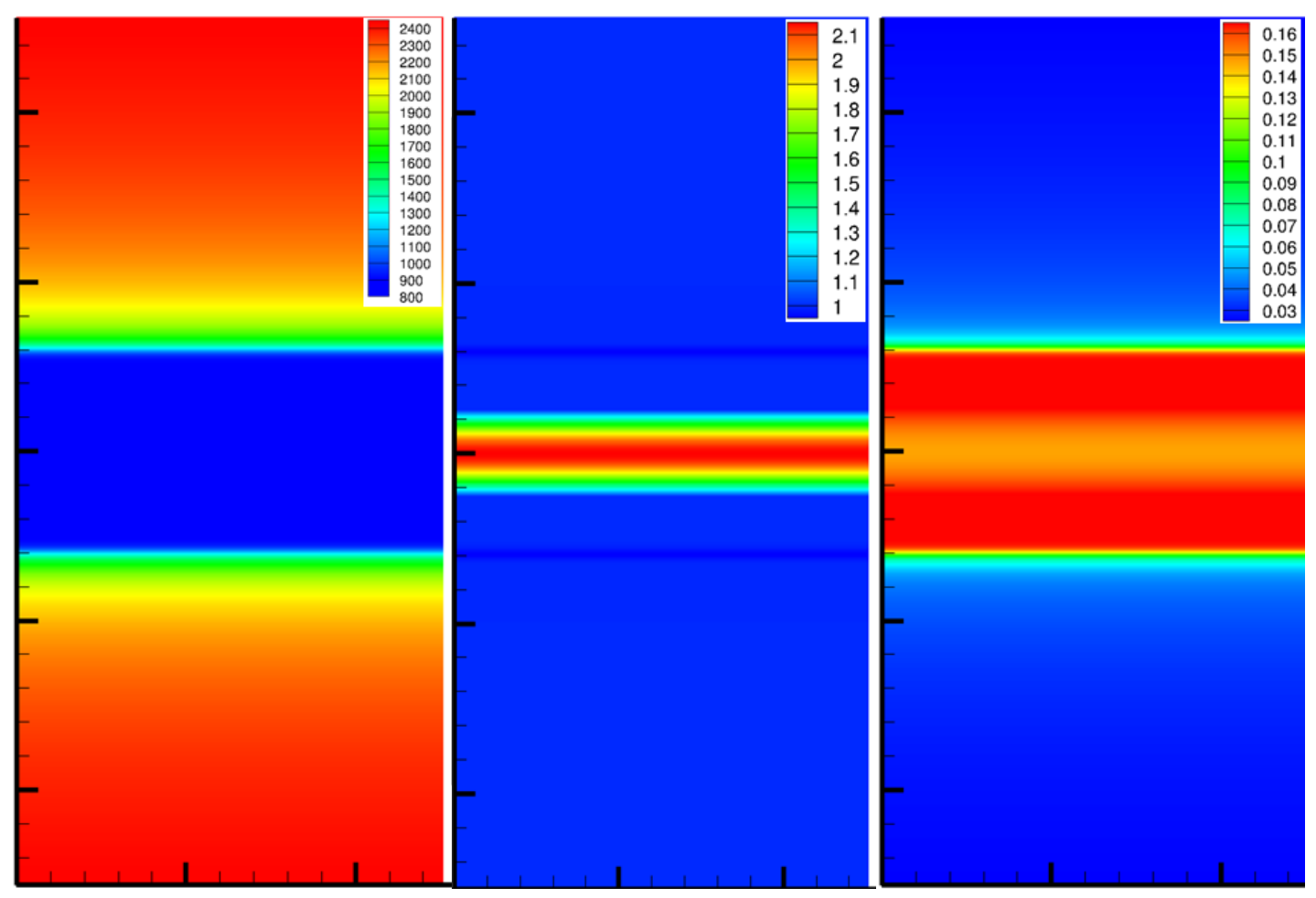

Figure 1: Initial contours of (a) temperature $(\mathrm{K})$, (b) equivalence ratio (c) $\mathrm{O}_{2}$ mass fraction

A freely propagating, laminar premixed flame simulation using CHEMKIN-PREMIX, at equivalence ratio 1.0 initializes the reacted jets. The temperature in the central reactant jet is set to be $900 \mathrm{~K}$. Syngas $\left(0.675 \mathrm{H}_{2}+0.225 \mathrm{CO}+0.1 \mathrm{~N}_{2}\right.$, molar) is the fuel used in the present investigation since it can be argued that it is representative of the reactants composition following initial pyrolysis of hydrocarbon fuels; a small amount of nitrogen was added to moderate combustion temperature and its spatial gradients, and thus to limit mesh fineness. Syngas oxidation kinetics was modeled by a skeletal mechanism including 29 reactions and 12 species $\left(\mathrm{H}_{2}, \mathrm{H}, \mathrm{O}, \mathrm{OH}, \mathrm{H}_{2} \mathrm{O}, \mathrm{O}_{2}, \mathrm{HO}_{2}, \mathrm{H}_{2} \mathrm{O}_{2}, \mathrm{CO}, \mathrm{CO}_{2}, \mathrm{HCO}\right.$ and $\left.\mathrm{N}_{2}\right)$ [22] derived from the $\mathrm{C} 1$ mechanism in [23]. The first five species are the lightest and most impacted by preferential diffusion. Initial conditions for the streamwise velocity are approximated using a hyperbolic tangent profile (peak velocity $312 \mathrm{~m} / \mathrm{s}$ ) with turbulent fluctuations superimposed to all three velocity components corresponding to $7.5 \%$ turbulence intensity. The bulk Reynolds number 
based on the jet height, $\mathrm{H}$, jet velocity, $\mathrm{U}$, and viscosity at the initial jet temperature is $\mathrm{Re}_{\text {bulk }}=$ $\rho \mathrm{UH} / \mu=8000$. Based on turbulence intensity, the initial turbulent Reynolds number imposed is $\mathrm{Re}_{\text {Turb }}=\rho u^{\prime \prime} \mathrm{H} / \mu=600$ and $\mathrm{Re}_{\lambda=110}$, where $\mathrm{Re}_{\lambda}$ is the Taylor scale Reynolds number.

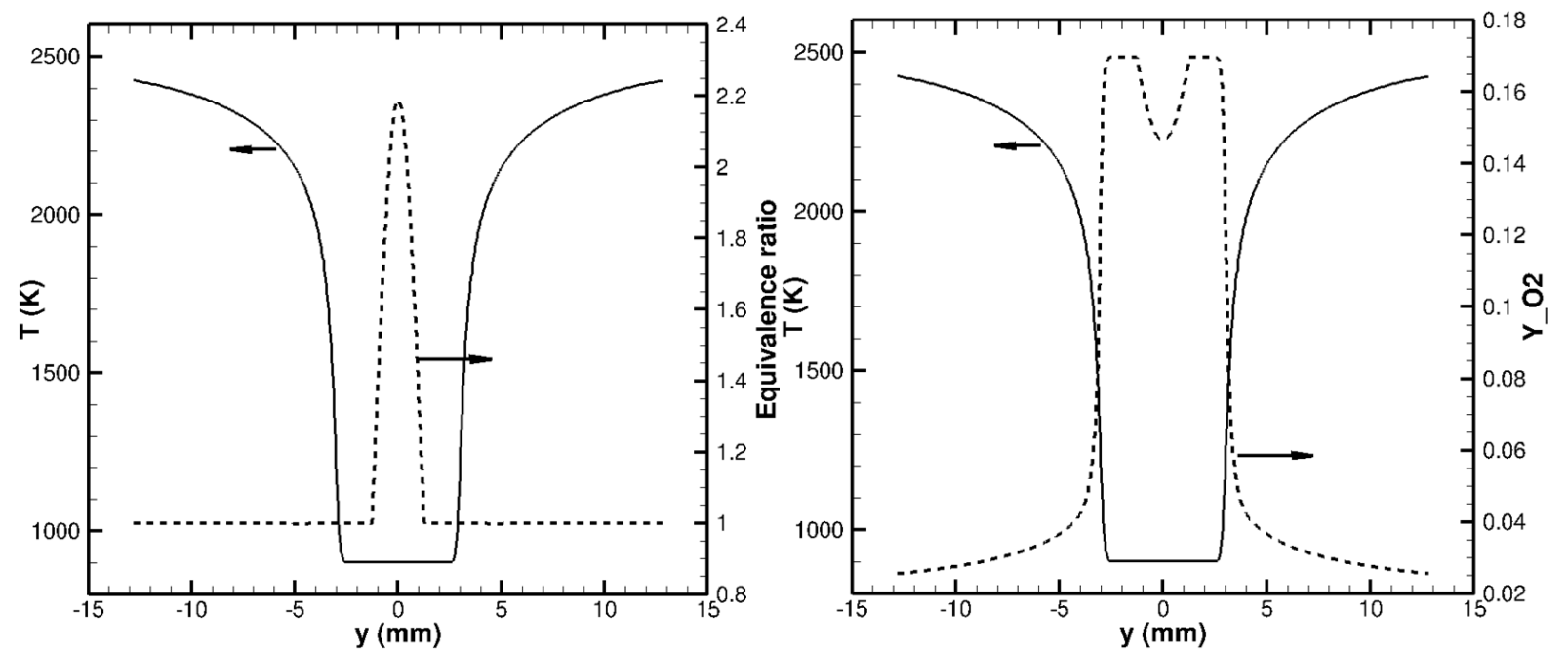

Figure 2: Initial profiles of (a) temperature and equivalence ratio (b) temperature and Oxygen mass fraction.

Figures 2 shows the initialized profiles on the mid-plane as a function of the transverse coordinate. It is important to notice the unique initialization used in this study since it may have some implications on the results reported later in this paper. The two premixed flames are initialized slightly outside the shear layer (flat temperature region); composition and temperature of these two flames correspond to those of a stoichiometric mixture. The equivalence ratio $(\phi)$ is initialized within the inner $1 \mathrm{H}$ region inside the unburnt portion of the flames as shown in Figure 2 (right). Similarly, turbulent fluctuations were imposed only in the unburnt portion of the flame, to ensure that turbulence interacts with the flame only after fluctuations develop into realistic turbulence.

\subsection{Domain, grid size and boundary conditions}

The extent of the domain is $5 \mathrm{H}$ in the streamwise $(x)$ direction, $10 \mathrm{H}$ in the crossstream/transverse $(\mathrm{y})$ direction and $3 \mathrm{H}$ in the spanwise (z) direction. Pope [24] argues that the large anisotropic scales of the order of $I_{0}$, are approximately 6 times larger than the $I_{E I}$ where $I_{E I}$ is a measure of the largest length scale in the inertial sub-range. Therefore setting $\mathrm{I}_{0}=\mathrm{H}$ the DNS domain size in which the flame propagates in a statistical sense spans more than $5 I_{E I}$ in the $\mathrm{x}$-direction and more than $10 \mathrm{I}_{\mathrm{El}}$ in the transverse direction. The Kolmogorov scale $(\eta)$ is estimated using $\eta=N_{\eta}{ }^{*} \mathrm{H} \operatorname{Re}_{L}{ }^{-3 / 4}$ as $30 \mu \mathrm{m}$ for the integral Reynolds number $=600$. Here $\mathrm{N}_{\eta}$ is a numerical constant set to 1.4. CHEMKIN-PREMIX solutions for the initial flame structure yield a measure of the species profile thickness, the thinnest $(240 \mu \mathrm{m})$ being that of $\mathrm{HO}_{2}$. That suggested a $30 \mu \mathrm{m}$ DNS resolution is sufficient to resolve all species and velocity scales. This also indicated that the initial Kolmogorov scales are smaller than species profile thickness and hence the flame is expected to be in the distributed reaction regime, where turbulence penetrates the diffusion (preheat) zone and may interact with reaction zone kinetics. 
The boundary conditions used in the simulations are periodic in both streamwise and spanwise directions. In the transverse (y) direction, outflow boundaries allow pressure waves to leave the domain. To this purpose, Navier-Stokes Characteristic Boundary conditions (NSCBC) [25] are used to simulate the partially reflecting outflows in the transverse direction.

\subsection{CFD Codes}

To verify that in turbulent flames molecular diffusion is not a negligible high-order effect, DNS were performed using two CFD codes, LESLIE and S3D briefly described below.

(i) LESLIE is developed by S. Menon and co-workers at Georgia Institute of Technology [26]. It is a multi-block structured, finite volume solver for the fully compressible Navier-Stokes equations solved in generalized coordinates. The basic numerical scheme for integrating the discretized equations is second-order in time and includes a predictor/corrector scheme that is second or fourth order accurate in space. A thermally perfect gas model accounts for the temperature dependent specific heat capacities of all species. Finite-rate chemistry was integrated using a fourth-order accurate Runge-Kutta ODE integrator. The CANTERA package supplies the molecular diffusion models.

(ii) S3D is developed by J. Chen and co-workers at Sandia National Laboratories [27]. S3D solves the finite difference form of fully compressible Navier-Stokes equation on a single block rectangular Cartesian domain. This code is $6^{\text {th }}$ order accurate in time and $8^{\text {th }}$ order accurate in space with $10^{\text {th }}$ order filtering of spurious numerical modes. A thermally perfect gas model accounts for the temperature dependent specific heat capacities of all species. Finite-rate chemistry is integrated using a fourth-order accurate Runge-Kutta ODE integrator. The CHEMKIN transport package supplied the molecular diffusion models.

Both codes include the Soret effect, thermal conductivity and viscosity with the same physical accuracy [28]. However, pressure-driven diffusion (the second term on the RHS of equation (1) in Appendix A) was neglected, both to save time and because it can be argued that the $\nabla \ln (p)$ contribution is small in the low-subsonic regime simulated here. This may not be true in highly compressible flows, close to supercritical pressure, and in liquids.

The purpose of using two solvers is twofold:

(i) since there are no experimental data to compare with CFD solutions, comparing solutions from two different codes serves as an indirect validation of simulations;

(ii) to ascertain whether multi-component diffusion effects can be detected only by the higher order CFD methods such as the ones used in S3D code, or by $2^{\text {nd }}$ accurate codes as well (prevalent in research and commercial codes). In other words, if S3D detected diffusion effects, but LESLIE could not, it would confirm the widely held opinion that in turbulent combustion molecular diffusion is a high-order effect that can be neglected.

\section{Results}

4.1 DNS validation of LESLIE and S3D results using the Mixture-Averaged Diffusion model

The purpose of the initial simulations was to validate and evaluate the performance of LESLIE and S3D. DNS of partially premixed reacting mixing layers were conducted independently and 
the results are compared below. Both codes used the same mixture-averaged diffusion (MAD) model (see Appendix A for equations). Even though this model does not require calculating $D_{\mathrm{i}}$, it still involves expensive computation of binary diffusion coefficients for each species pair.

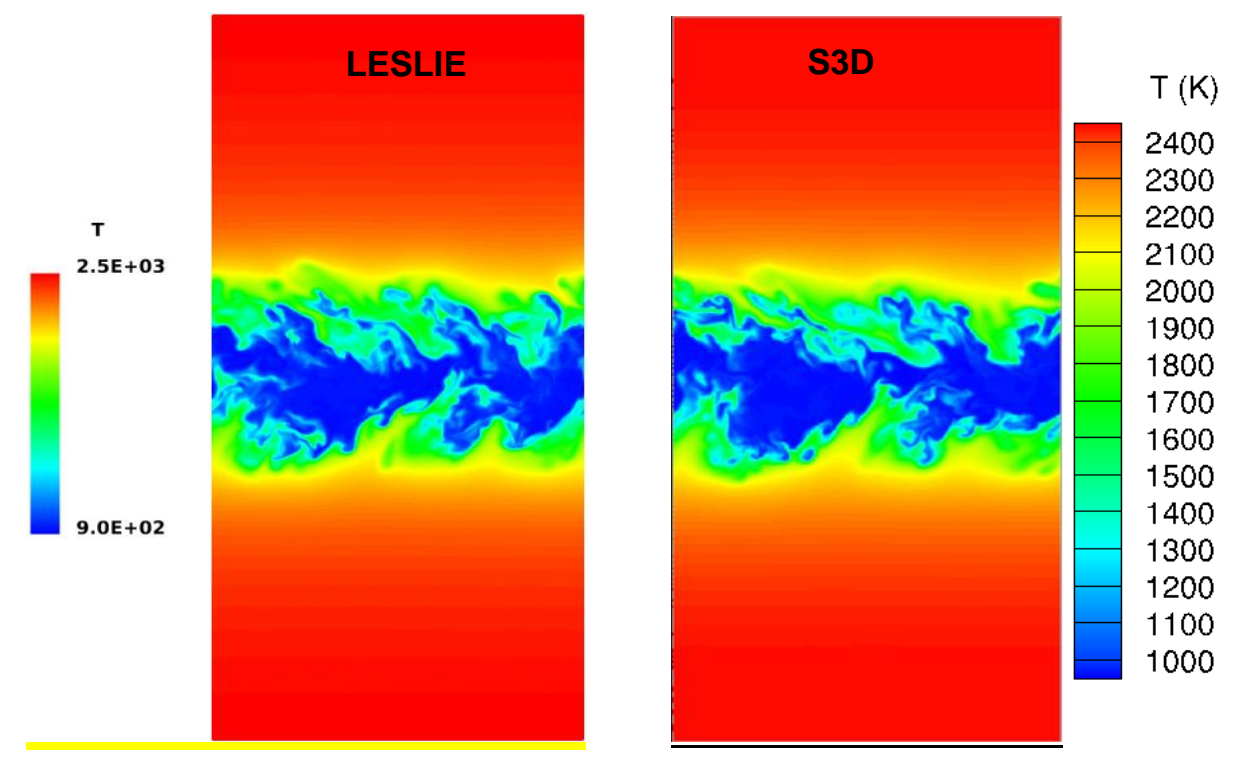

Figure 3: Temperature maps at an arbitrary instant from LESLIE (left) and S3D (right)

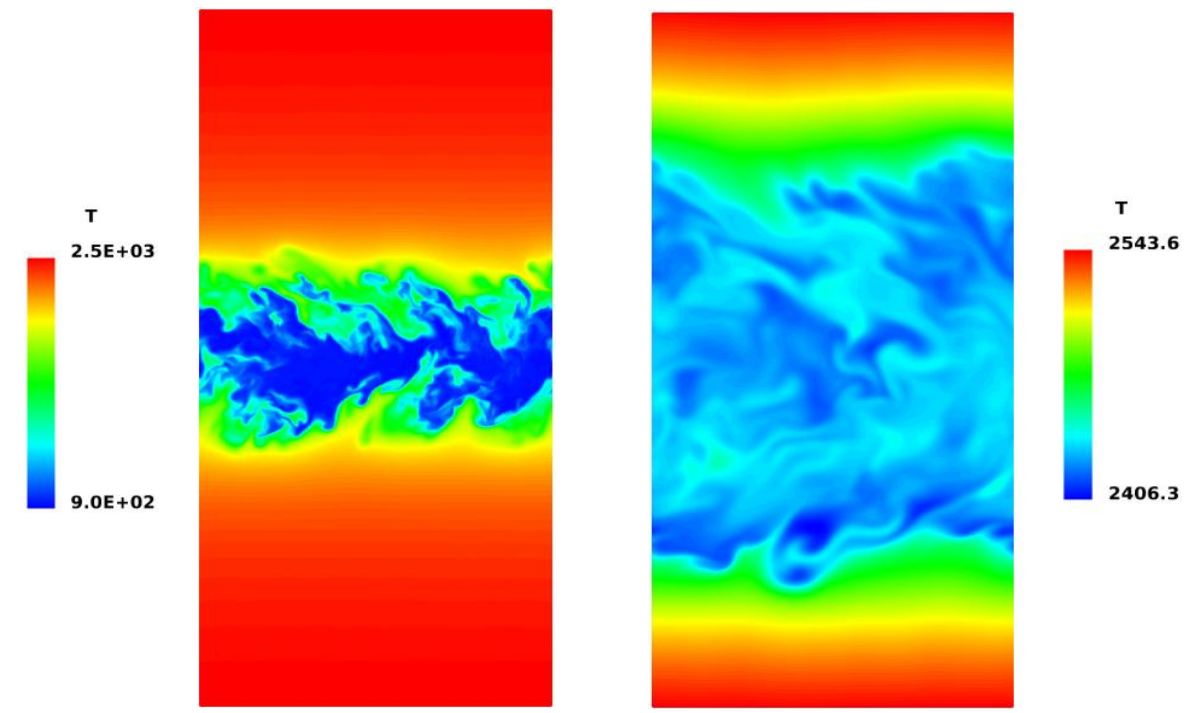

Figure 4: Temperature maps $(K)$ at early and late times predicted by LESLIE using the Mixture Averaged Diffusion model

Figure 3 shows side-by-side instantaneous temperature maps on the spanwise mid-plane $z=$ $1.5 \mathrm{H}$, obtained with the two CFD codes at an instant when shear layers have begun to interact vigorously with the flame. Fine scale turbulent structures are seen riding on two larger coherent structures. Peak temperature, length scale magnitude and location look very similar; minor differences are attributed to the slightly different time instants at which the images were taken and to the different numerical schemes. Overall, the qualitative agreement appears reasonable. 
Since the results from the two codes are very close, only LESLIE color maps at two nondimensional times $t^{*} \equiv t / T_{L}=2 / 3$ (left) and 2 (right) are reported in fig. 4 . Here $T_{L}$ refers to the large eddy turn-over time defined as $T_{L}=L / u^{\prime \prime}$, where $L$ is the integral scale, taken to be the initial jet height $\mathrm{H}$, and $\mathrm{u}^{\text {" is }}$ the initial turbulent fluctuation. At time $\mathrm{t}^{\star}=2$ the flame has nearly reached the $y= \pm 5 \mathrm{H}$ boundaries and is beginning to feel the effect of the outflow boundary. Simulations were stopped when the flame reaches closer to the outflow boundary $\left(\sim t^{\star}=2\right)$ to prevent statistics from being influenced by outflow effects. As expected, the temperature contour maps in Figure 4 predict two flame fronts initially spreading inwards from the two shear layer containing high temperature products toward the fuel rich colder central jet. Growth of the mixing layers is accelerated by turbulent mixing with the central core that still contains unreacted fuel and air.

Qualitative contour maps of species $\left(\mathrm{CO}, \mathrm{H}_{2}, \mathrm{O}_{2}, \mathrm{H}_{2} \mathrm{O}, \mathrm{OH}, \mathrm{H}, \mathrm{O}, \mathrm{HO}_{2}, \mathrm{HCO}, \mathrm{CO}_{2}\right.$, and $\mathrm{H}_{2} \mathrm{O}_{2}$ ) as the flame propagates from the lean, hot shear layers toward the central rich jet, and back, are shown in figs. 6 to 9. The maps show the effect of the interplay among syngas/air kinetics, turbulent and molecular transport of heat and mass. Note that the color maps at early and late times have different ranges: for instance, in Figure 4, the late time temperature (right) near the $\mathrm{y}= \pm 5 \mathrm{H}$ boundaries is higher than at the early time (left). Thus the evolving temperature mixing layers thicken and react while the energy released is transported inwards (initially) and outwards (at later times).

Although contour maps are qualitative and their ranges differ, some observations are worth of note.

Observation 1: Even though the contour maps of all the species shown in figs. $6-9$ show a monotonic behavior from the center-line to the outer boundaries, there is a peculiar behavior for $\mathrm{H}_{2}, \mathrm{O}_{2}$ and $\mathrm{H}_{2} \mathrm{O}$ species. To analyze this further, line plots of these species and equivalence ratio at three time instants are shown in fig. 5. These plots show that initially the flames move inward towards each other while, simultaneously, the equivalence ratio variation spreads out in the transverse direction as shown by the mean transverse profile of equivalence ratio (left) and $\mathrm{O}_{2}$ (right) and their time evolution. While the flames continuously transform the unburnt mixture into products, the equivalence ratio keeps spreading out. So at a given location and time, say y $\sim 6 \mathrm{~mm}$ at $1.5 \mathrm{~T}_{\mathrm{L}}$, the mixture is close to burnt conditions but corresponding to an equivalence ratio $\sim 1.15$, which is noticeably higher than the equivalence ratio of the mixture at the edges of the domain. This results in a "local maximum" of $\mathrm{O}_{2}$; said otherwise, moving outwards in the transverse direction the $\mathrm{O}_{2}$ mass fraction goes up before going down. The same trend is apparent in the mean $\mathrm{H}_{2} \mathrm{O}$ variation, see fig. 7 .

Note that the higher equivalence ratio near the centerline is responsible for the non-monotonic profiles of $\mathrm{H}_{2} \mathrm{O}$ and $\mathrm{O}_{2}$, but not for those of $\mathrm{H}_{2}$ or temperature (Figures 4 and 6). A simple explanation may be based on the choice of initial $\Phi$ profiles. Richer mixtures imply a higher $\mathrm{H}_{2}$ mass fractions and lower temperatures, slowing kinetics capable of altering the monotonous distribution of $\mathrm{H} 2$ from the central jet to the reacted boundary.

A second conjecture is that the mild peak of $\mathrm{H}_{2} \mathrm{O}$ is due to the simultaneous inward transport of $\mathrm{O}_{2}$ and outward transport of $\mathrm{H}_{2}$; a third is the rapid consumption of $\mathrm{O}_{2}$ in the central zone 
depressing its concentration more rapidly than the effect of diffusion from the boundaries; $\mathrm{O}_{2}$ at the boundaries remains almost constant, so during the time simulated a mild local maximum may form.
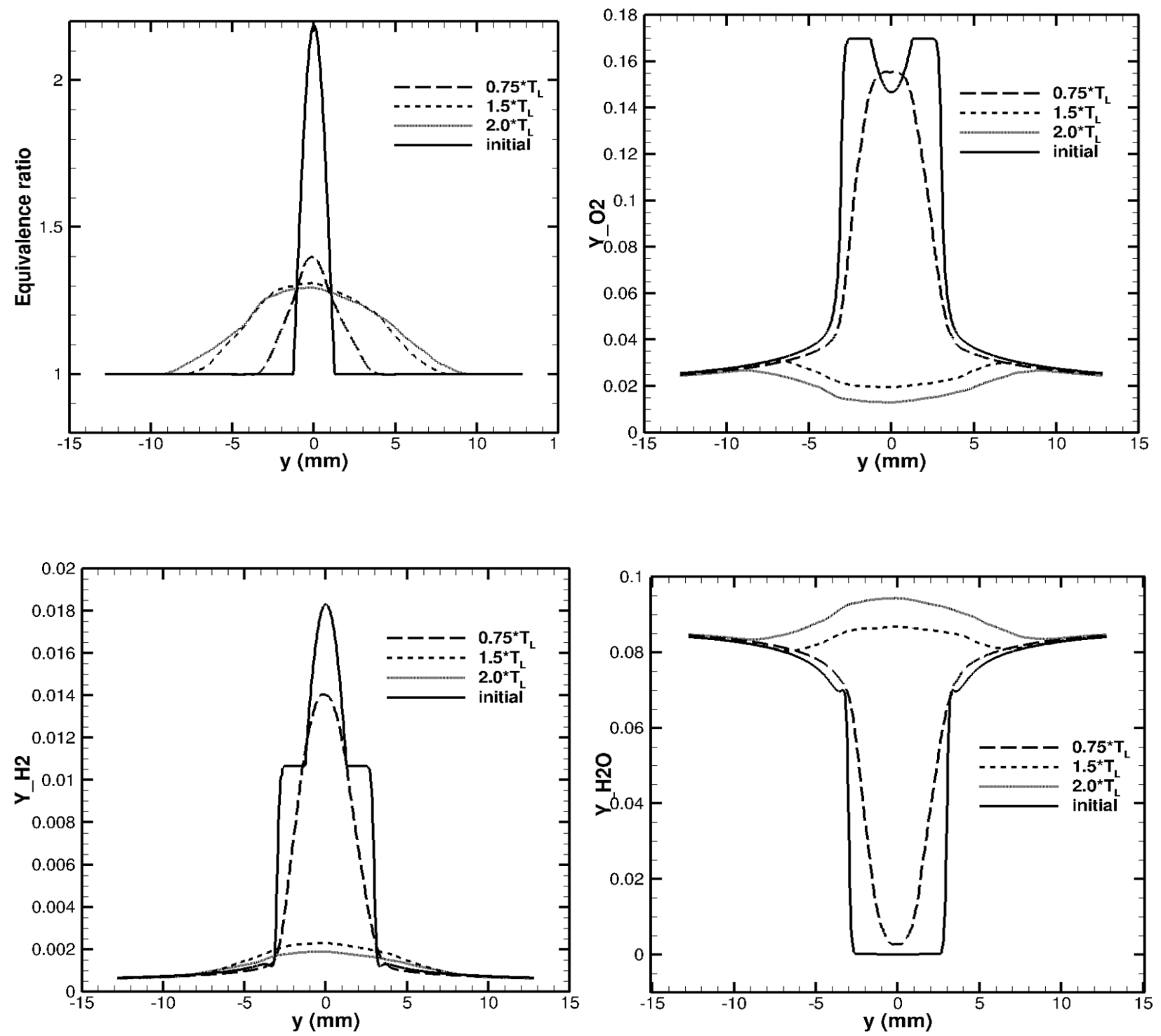

Figure 5: Evolution of equivalence ratio and mass fraction of $\mathrm{O} 2, \mathrm{H} 2$, and $\mathrm{H} 2 \mathrm{O}$ species at three instants. LESLIE using Mixture Averaged Diffusion model

Observation 2: $\mathrm{CO}_{2}$ behaves as other species, but its percentage change, estimated from the evolution of peak concentrations, is appreciably lower than that of $\mathrm{H}_{2} \mathrm{O}$. This is consistent with the observation in [7] that faster diffusion of lighter species out of the reaction zone may enrich it with heavier (slower) molecules such as $\mathrm{CO}_{2}$. 


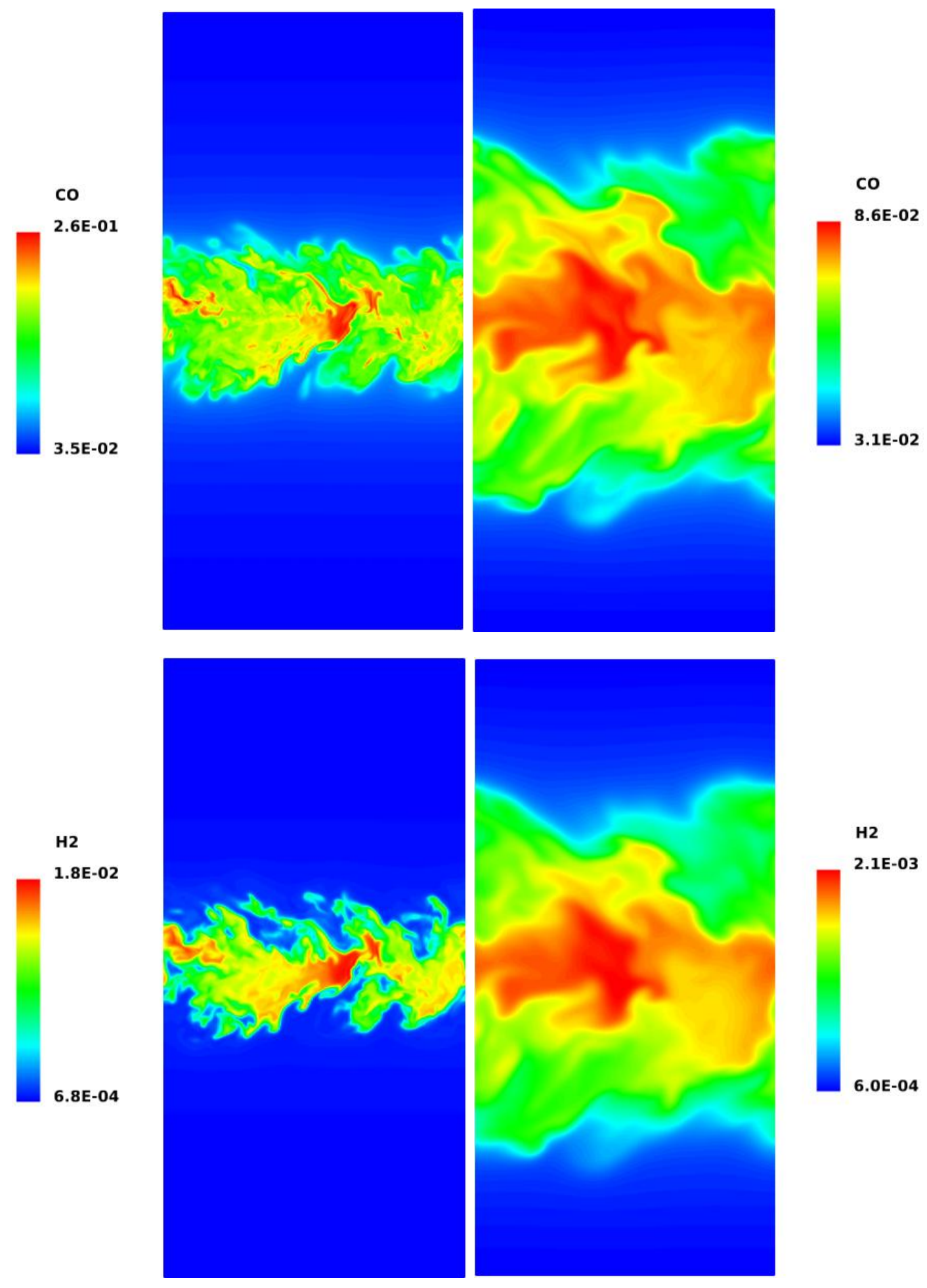

Figure 6: $\mathrm{CO}$ and $\mathrm{H}_{2}$ mass fractions at early and late times. LESLIE Mixture Averaged Diffusion model 


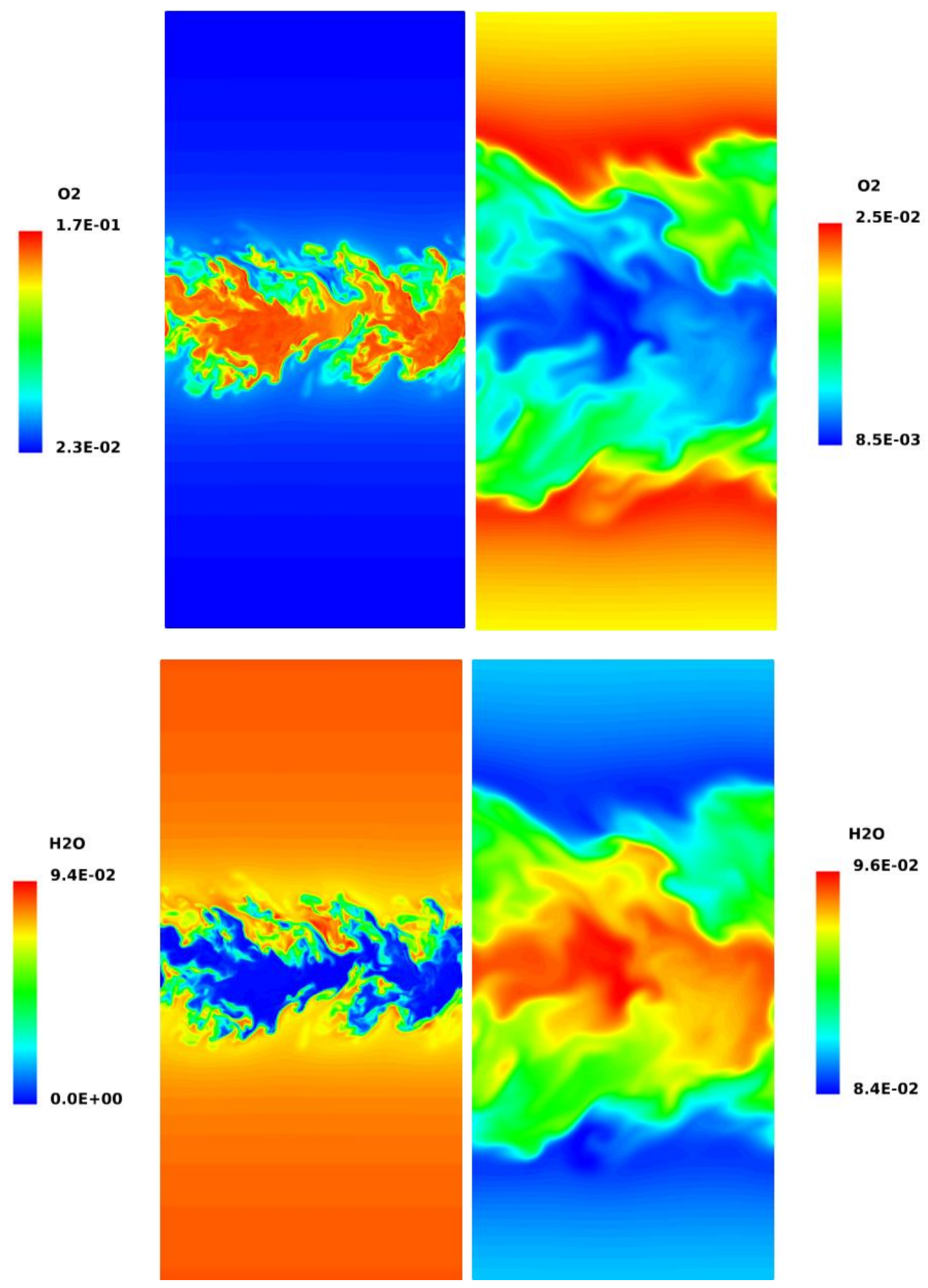

Figure 7: $\mathrm{O}_{2}$ and $\mathrm{H}_{2} \mathrm{O}$ mass fractions at early and late times. LESLIE Mixture Averaged Diffusion model 

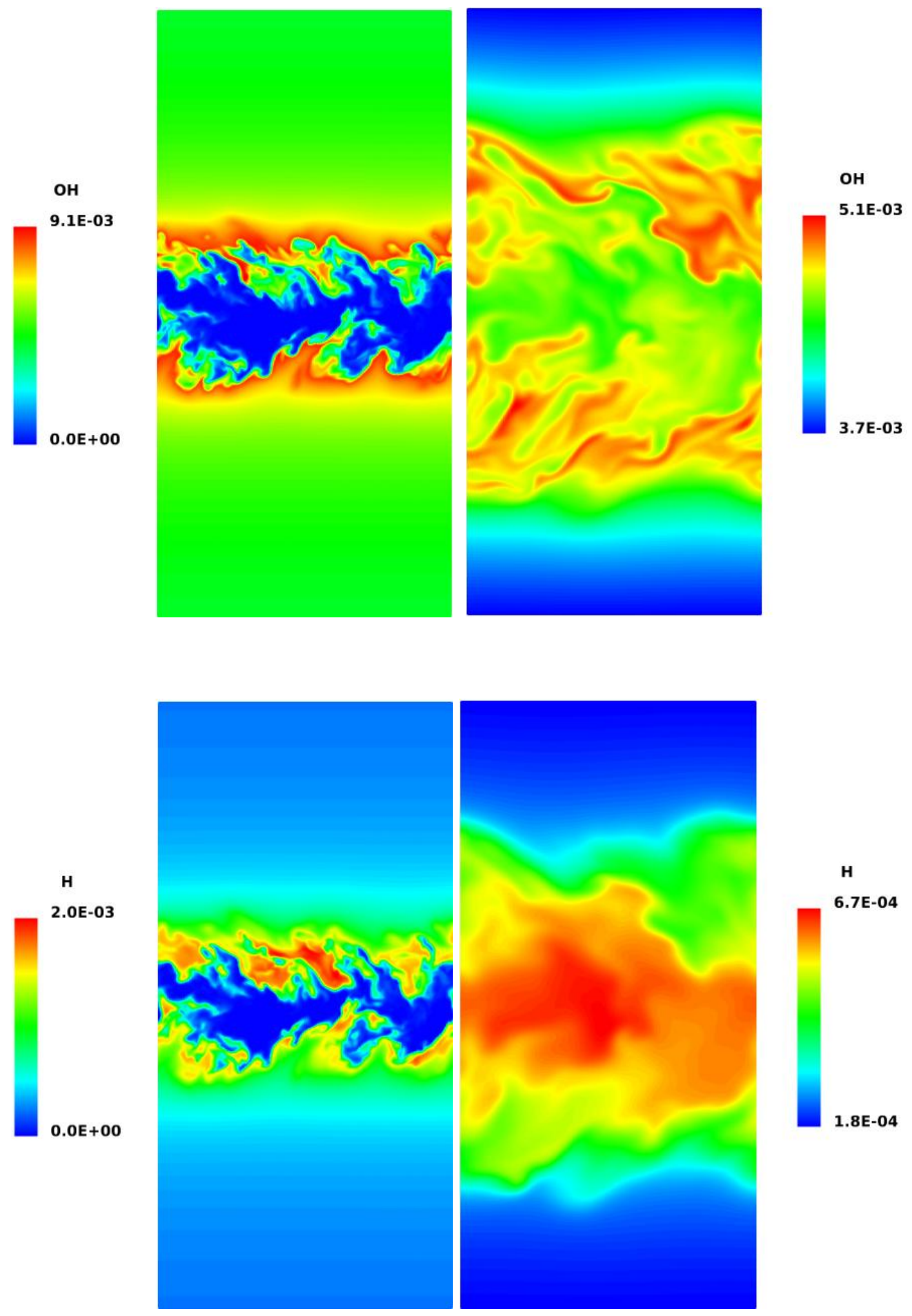

Figure 8: $\mathrm{OH}$ and $\mathrm{H}$ mass fractions at early and late times. LESLIE Mixture Averaged Diffusion model 


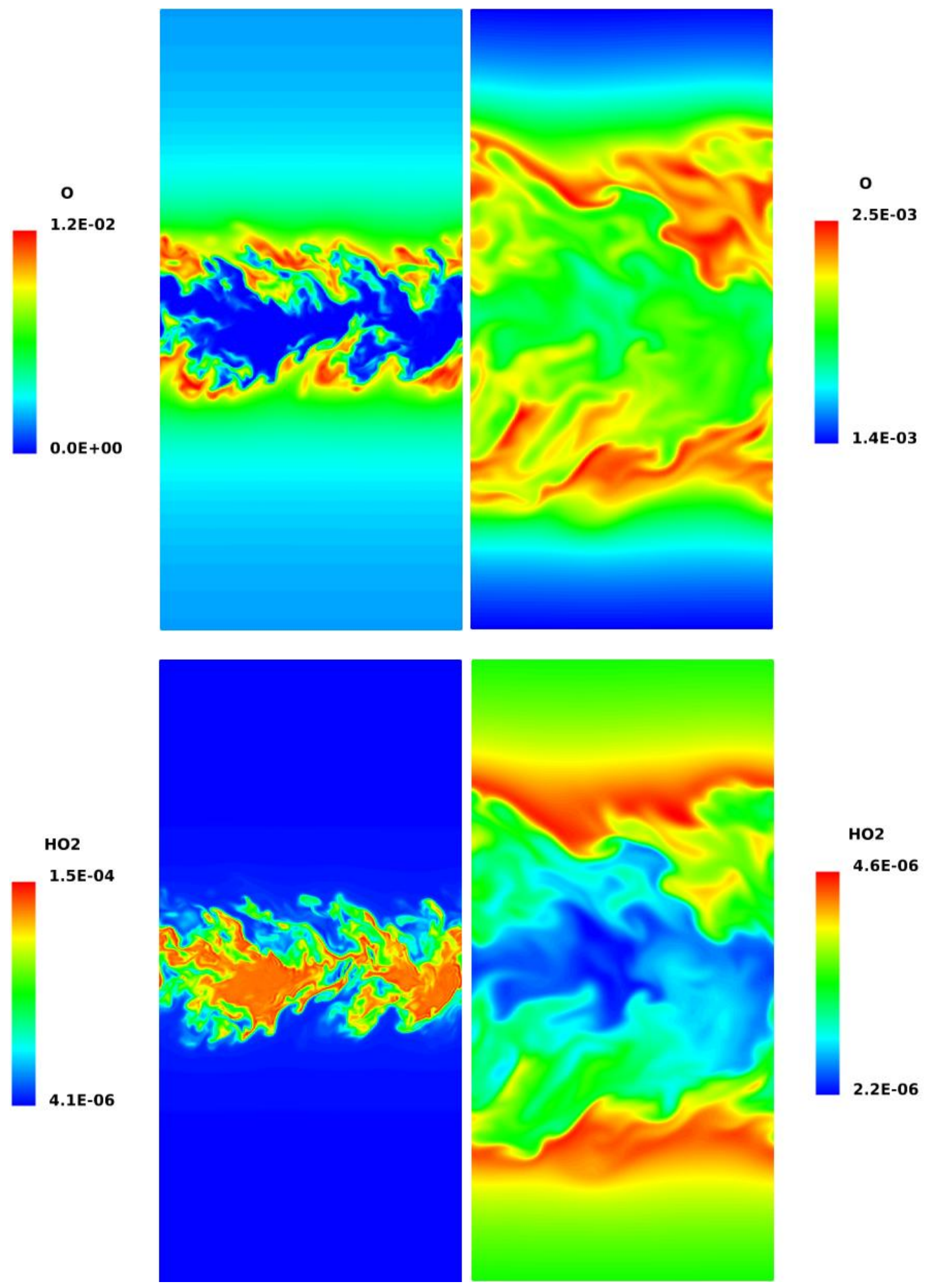

Figure 9: $\mathrm{O}$ and $\mathrm{HO}_{2}$ mass fraction at early and late times. LESLIE Mixture Averaged Diffusion model 


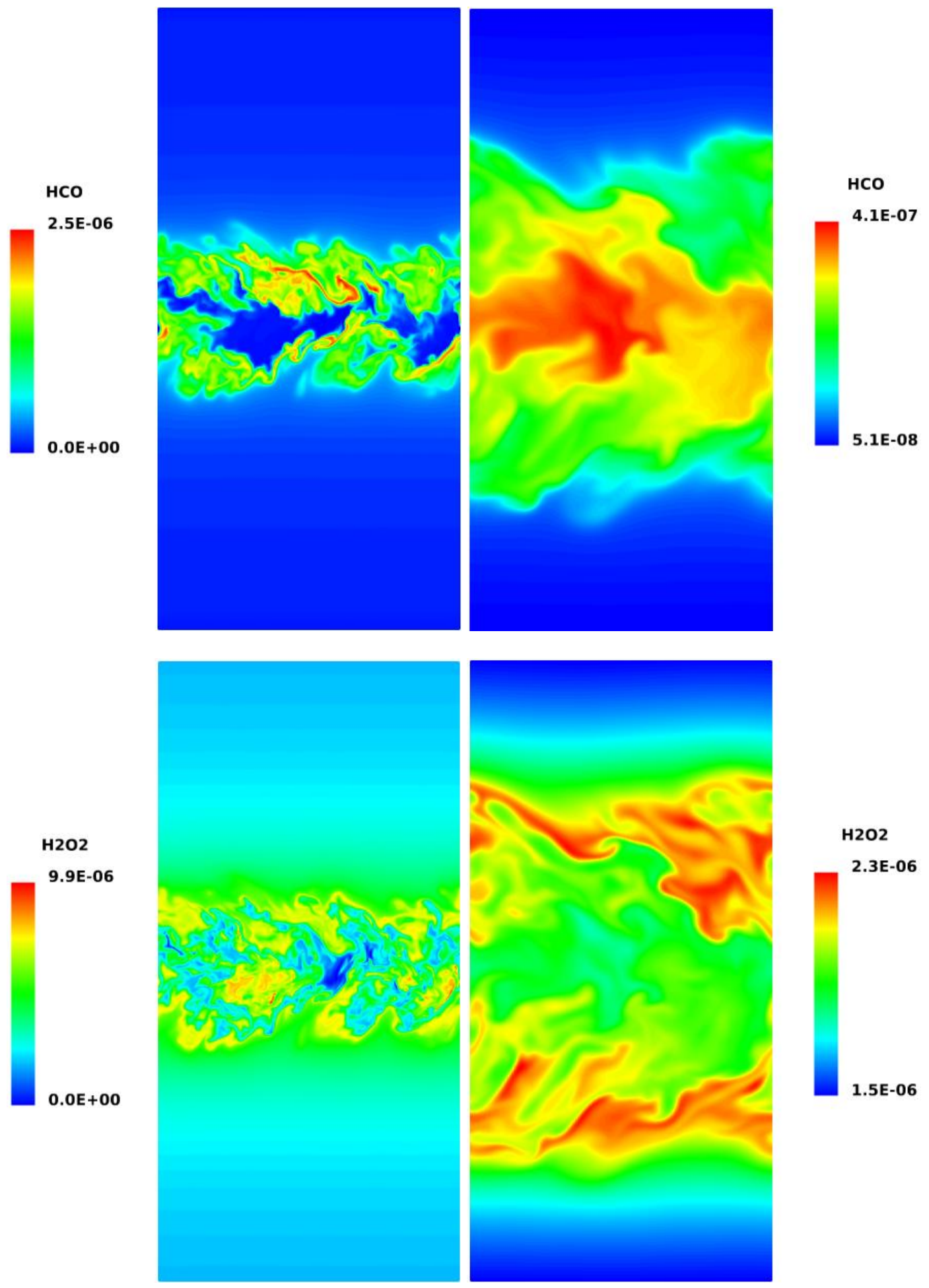

Figure 10: $\mathrm{HCO}$ and $\mathrm{H}_{2} \mathrm{O}_{2}$ mass fractions at early and late times. LESLIE using Mixture Averaged Diffusion model 


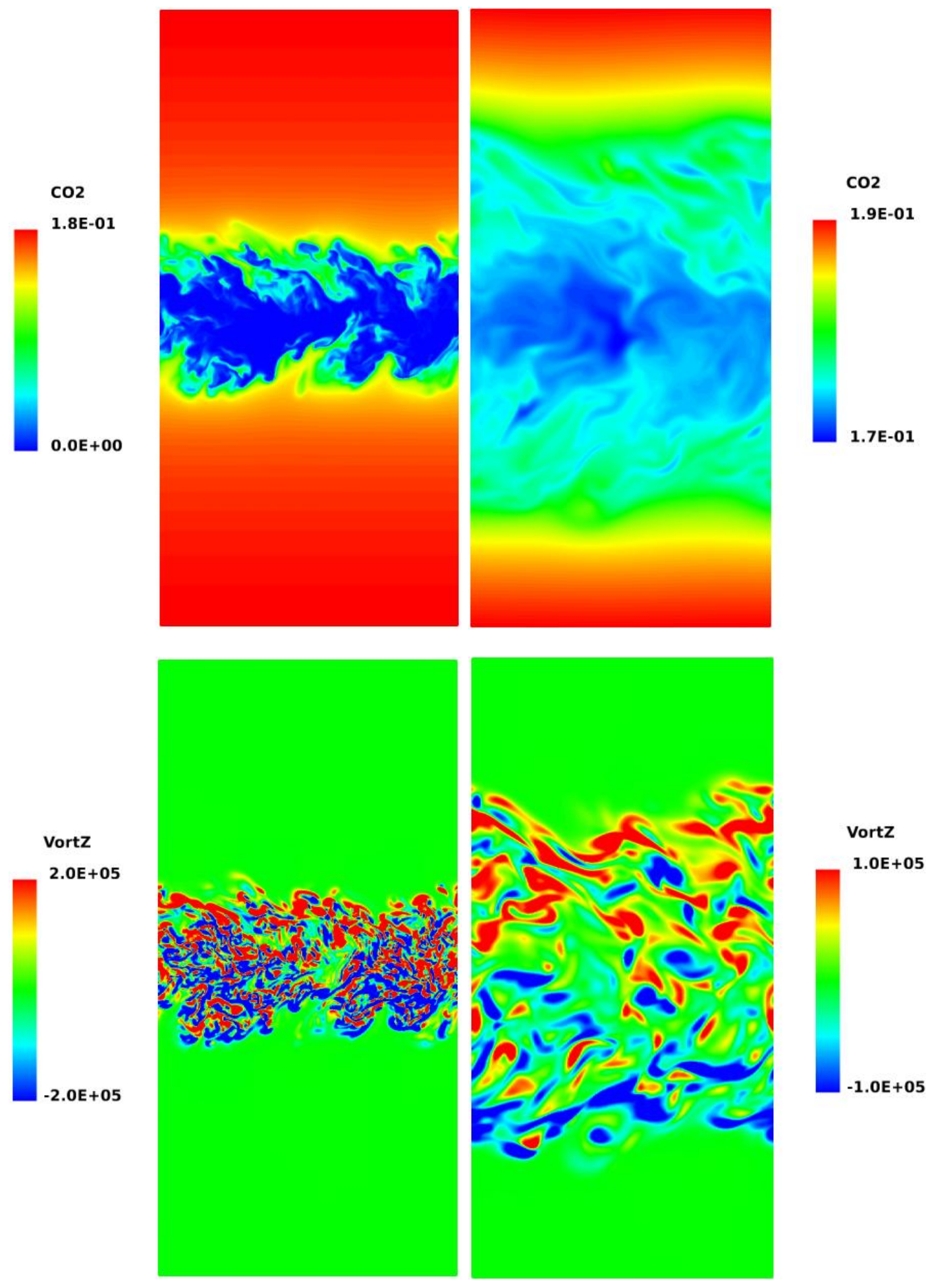

Figure 11: $\mathrm{CO}_{2}$ mass fraction and spanwise Vorticity component $(\mathrm{Hz})$ at early and late times. LESLIE using Mixture Average Diffusion model 
Observation 3: $\mathrm{CO}_{2}$ and $\mathrm{H}_{2} \mathrm{O}$ should be transported equally by turbulence; instead their transverse $(\mathrm{y})$ gradients are oriented in opposite directions. A possible explanation is preferential diffusion of $\mathrm{H}_{2} \mathrm{O}$ toward the hotter central region; an alternative kinetic explanation has been suggested based on the much larger formation rate of $\mathrm{H}_{2} \mathrm{O}$ than $\mathrm{CO}_{2}$ in the central jet region due to stoichiometry. Both could justify the difference between the trends of the two major products.

Observation 4: Similarly, the sharp $\mathrm{O}, \mathrm{OH}$ and $\mathrm{H}$ gradients present at early times disappear at late times, presumably due to recombination to water and $\mathrm{CO}_{2}$, but somewhat more slowly than the heavier $\mathrm{H}_{2} \mathrm{O}_{2}$ and $\mathrm{HCO}$.
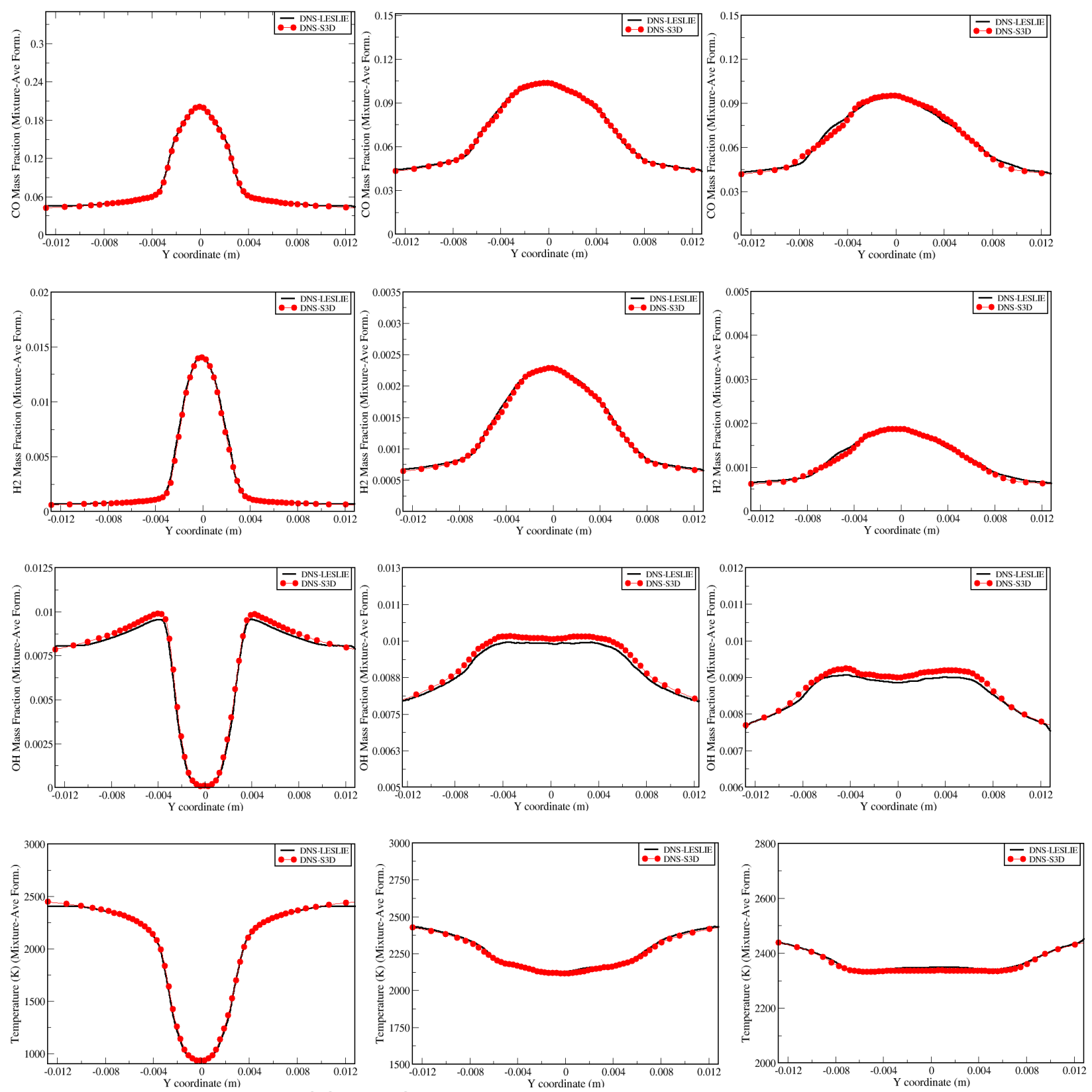

Figure 12: Top to bottom: $\mathrm{CO}, \mathrm{H}_{2}, \mathrm{OH}$, and temperature profiles. Left to right: $\mathrm{t}^{\star}=2 / 3,4 / 3$ and 2 . Black, LESLIE; red, S3D. Mixture Averaged Diffusion model. 
Overall, it can be deduced from these images that turbulence and kinetics alone cannot completely account for differences in species distribution, and that additional physics, e.g., molecular diffusion, plays an equally important role as well.

Figure 12 shows profiles of $\mathrm{CO}, \mathrm{H}_{2}, \mathrm{OH}$, and temperature at the three time instants along the transverse y direction. Since these are temporal mixing layers, all quantities plotted have been averaged over the streamwise $(x)$ and spanwise $(z)$ directions so that the statistical time evolution is present only in the $(y)$ direction, the main direction of interest: on a $x-z$ transverse plane, every point $(x, z)$ is treated as a sample and used in averaging. Overall the solutions obtained with the two codes are in very good agreement. Comparisons of other species (not shown) are equally good: even trace species (e.g., $\mathrm{HCO}, \mathrm{OH}$ ) exhibit differences of $5 \%$ at most. Figure 12 supports the conclusion that, notwithstanding the difference in formal accuracy between LESLIE and S3D, both predict almost identical results. The conclusion is that both codes are capable of capturing equally well the turbulence-chemistry-diffusion interaction.

4.2 Comparison between Multi-Component-Diffusion and Mixture-Averaged-Diffusion simulations using LESLIE

It is recalled that the primary objective of this work is to assess the impact and fidelity of diffusion models in simulating turbulent combustion. Based on the confidence gained from comparing LESLIE and S3D results, LESLIE alone was chosen to compare mixture-averaged diffusion (MAD) with the full multi-component diffusion (MCD) model. Accordingly, the DNS of the partially premixed temporal mixing layer was repeated with this model, and the results were compared with those already obtained using mixture-averaged diffusion. Figure 13 shows the comparisons. This figure shows the averaged line plots of $\mathrm{CO}, \mathrm{H} 2, \mathrm{OH}, \mathrm{HCO}$ mass fractions and temperature at times $t^{*}=(2 / 3)$ and $(4 / 3)$. Results are shown only at these two times since the trend becomes clear in just one large eddy turn-over time; moreover, as the simulation progresses and the flame propagate towards the outflow boundary, solutions at the late time begin to be affected by the boundary conditions: Even though the results are physically correct, it complicates the task of interpreting the results, since it would be difficult to separate the effect of diffusion modeling from that of the boundary conditions modeling.

Surprisingly, fig.13 indicates that the solutions with MCD and MAD are in close agreement. The maximum difference between the two simulations is less than $2 \%$. Laminar flames simulations with MAD are known to predict results significantly different from MCD [19] [29], justifying to some extent why this result was unexpected. Even though convective transport is the same for all species, molecular transport and kinetic rates are different. Since the three simultaneous mechanisms are interconnected, it is hard to pinpoint the reason for the almost identical flow fields using two diffusion models with different fidelity. In the absence of more data and analyses, two conjectures are possible: the first is that in the present simulation (and current set of conditions) the mixture-averaged diffusion model is as accurate as multicomponent diffusion; MCD effects may be prominent under a different set of conditions. To verify this hypothesis and demonstrate the sensitivity to initial and boundary conditions would require running DNS for various geometries and conditions which is an expensive proposition. The second conjecture is that molecular diffusion itself is insignificant compared to turbulent transport, and hence any difference among molecular diffusion models is too small to be of consequence. 

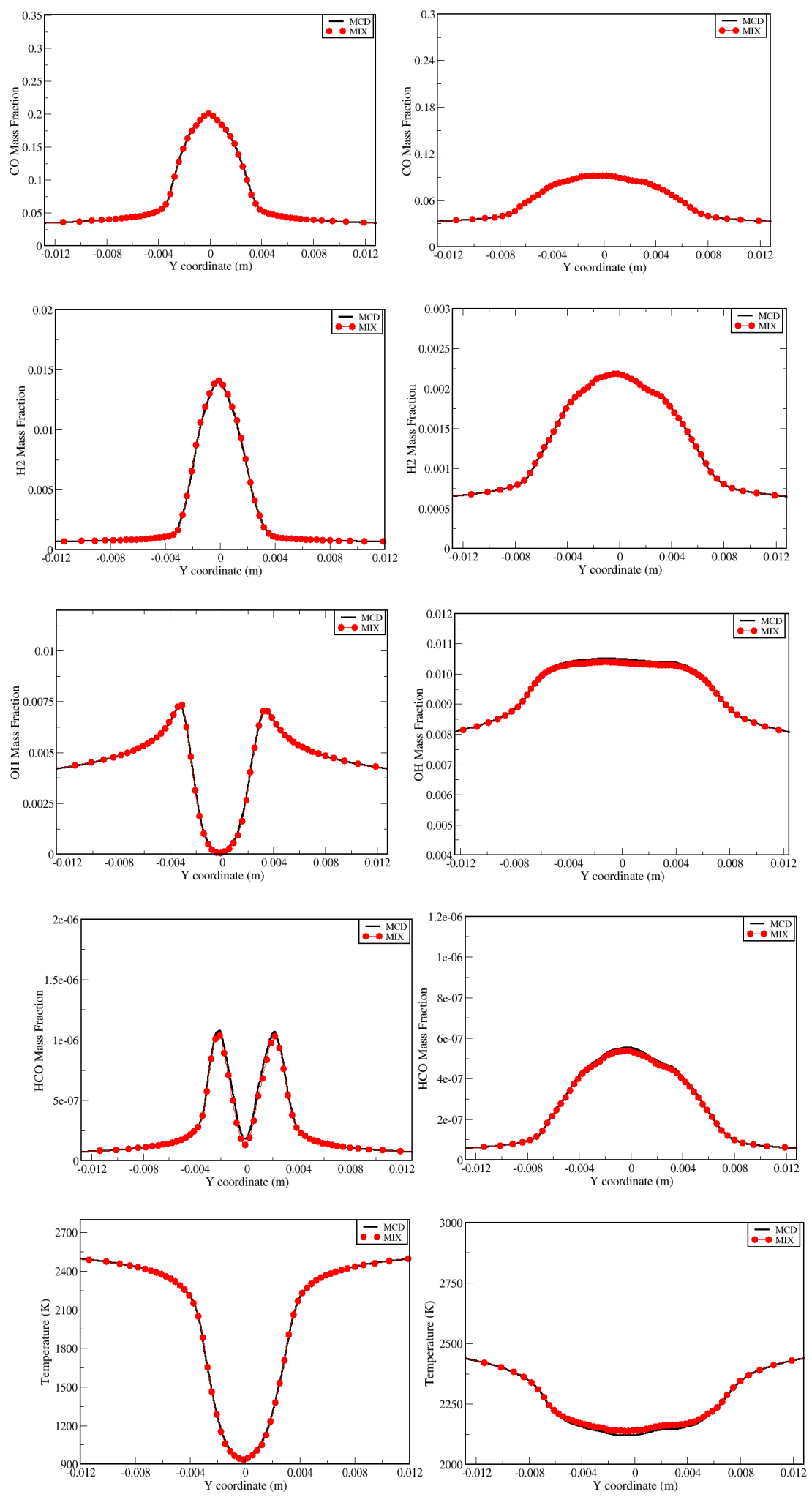

Figure 13: $\mathrm{CO}, \mathrm{H}_{2}, \mathrm{OH}, \mathrm{HCO}$ and temperature profiles predicted by LESLIE with mixtureaveraged diffusion (MIX) and with multi-component-diffusion (MCD). Left, $\mathrm{t}^{*}=2 / 3$; right, $\mathrm{t}^{\star}=4 / 3$. 
To test this second conjecture, an additional simulation was performed with the simpler assumption of unity Lewis number $L e_{i}$ for all species. If molecular diffusion is truly insignificant in the presence of turbulence, unity Lewis number simulations would yield results similar to the higher fidelity MAD and MCD diffusion models.

\subsection{Comparison between MAD and constant Lewis number models}

In premixed laminar flames the Lewis number shapes flame structure and speed $\left(\mathrm{S}_{\mathrm{L}}\right)$ depending on whether stretch is $>0$ or $<0: S_{L}$ increases for positive stretch and Le $>1$, and decreases when Le $<1$. In turbulent flames convection transports equally all species: thus were a significant Lewis number effect observed, it would mean that diffusive and convective transports are comparable. Note that unity $L_{i}$ do not imply the heat and mass fluxes are the same, since temperature gradients may differ in different zones of the flame. Furthermore, Lewis numbers are far from constant in reacting flows; however, $L e=1$ is a popular and very economical assumption in performing LES and DNS, since it does not require calculating binary diffusion coefficients.

Figures 14 and 15 compare the transverse $(\mathrm{y})$ profiles of species and temperature predicted by DNS with Lewis numbers $=1$ and those obtained with mixture averaged diffusion model. Significant differences are evident, and these grow with time. In the following analysis it is assumed that $L e=1$ is physically less accurate than MAD and MCD: results obtained with these models are retained as the standard: thus results classified „higher" or "Jower" refer to this standard.

The $\mathrm{CO}$ and $\mathrm{CO}_{2}$ profiles in fig.14 at late time appear consistent with $\mathrm{O}_{2}$ and $\mathrm{OH}$ profiles. With either $\mathrm{Le}=1$ or MAD, lower (higher) $\mathrm{CO}$ is correlated to lower (higher) $\mathrm{OH}$ and $\mathrm{O}_{2}$ and higher (lower) $\mathrm{CO}_{2}$. (The $\mathrm{CO}+\mathrm{OH} \rightarrow \mathrm{CO}_{2}+\mathrm{H}$ is the preferred $\mathrm{CO}$ oxidation pathway at the atmospheric pressure of this simulation.) $\mathrm{HCO}$, the precursor of $\mathrm{CO}$ initially present in the two high temperature jets, albeit a trace species, shows the same trend except in the two shear layers. $\mathrm{CO}$ and $\mathrm{H}_{2}$ are the only fresh reactants, but $\mathrm{H}_{2}$ does not behave like $\mathrm{CO}$ : its late time concentration is larger by 5 to $25 \%$ depending on $\mathrm{y}$, and that of $\mathrm{H}$ by up to $60 \%$, see Figure 15 The $\mathrm{H}_{2} \mathrm{O}$ fraction in Figure 14 is everywhere larger except in a small region centered around $\mathrm{y}=0$. It appears that $\mathrm{H}, \mathrm{OH}$ and $\mathrm{H}_{2}$, substantially lighter than all other species, are transported differently than heavier species like $\mathrm{CO}$ and $\mathrm{CO}_{2}$. An exception is $\mathrm{O}$, see Figure 15 , but in the mixing of a rich syngas flame and high temperature products even fast $\mathrm{O}$ atoms will collide with, and rapidly oxidize $\mathrm{H}$ - and $\mathrm{C}$-containing species.

This interpretation of the results implies

1. turbulent transport is significantly affected by the diffusion model;

2. a simple explanation based on diffusion transporting lighter species differently than heavier species

3. faster transport of $\mathrm{OH}$ and $\mathrm{H}$ produces more $\mathrm{CO}_{2}$ and higher temperatures.

Thus this test case shows not only that the effect of molecular diffusion is not negligible compared to turbulent convection, and that its magnitude depends on the molecular diffusion model, but also that $\mathrm{Le}_{\mathrm{i}}=1$ favors faster transport and, ultimately, faster kinetic and heat release rates. In this sense, molecular transport may interact with kinetic pathways. 

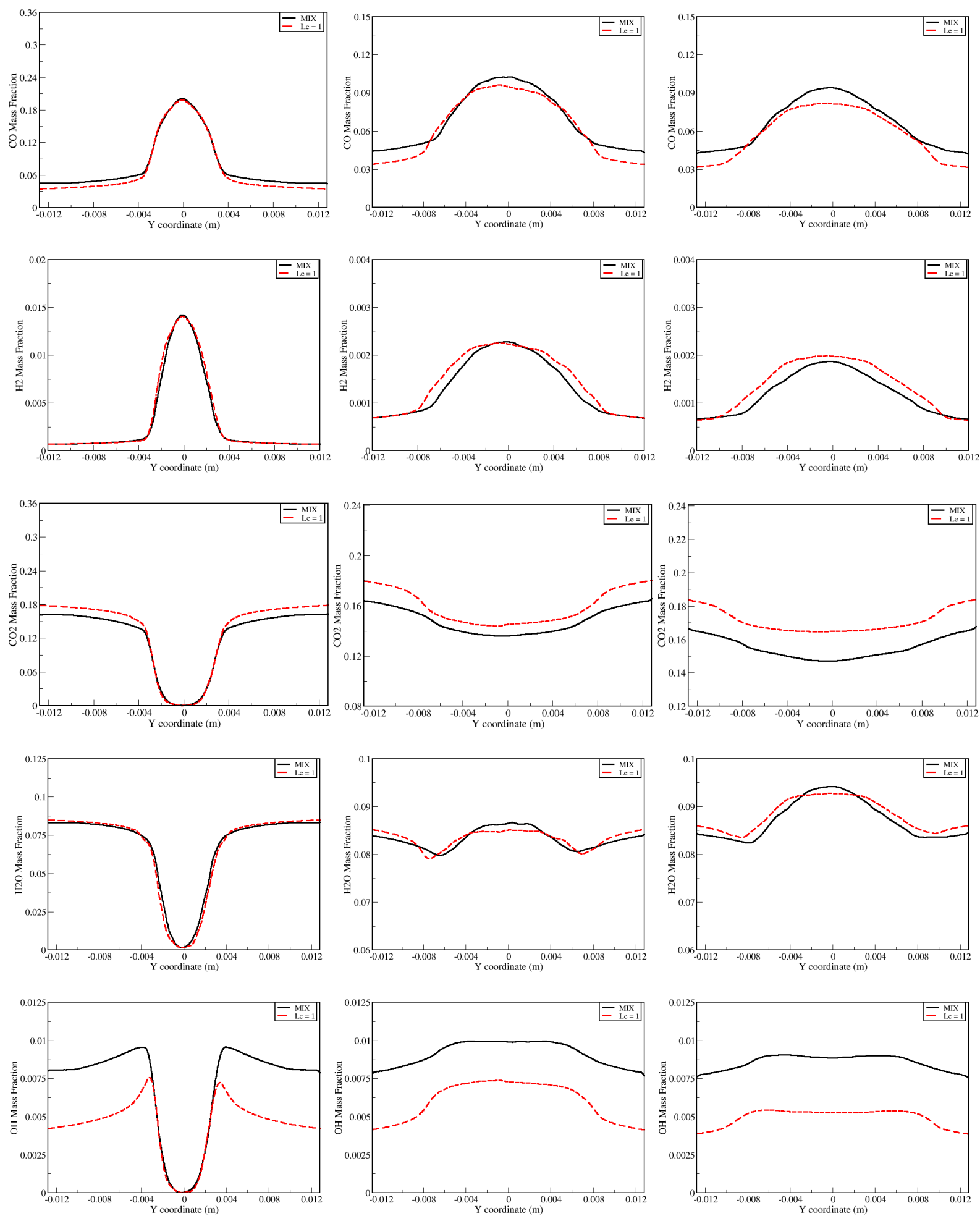

Figure 14: $\mathrm{CO}, \mathrm{H}_{2}, \mathrm{CO}_{2}, \mathrm{H}_{2} \mathrm{O}$, and $\mathrm{OH}$ mass fractions predicted by LESLIE with mixtureaveraged diffusion (MIX) and with constant Lewis number $=1$. Left to right: $t^{*}=2 / 3,4 / 3$ and 2 . Black, MAD; red, Le=1 model. 

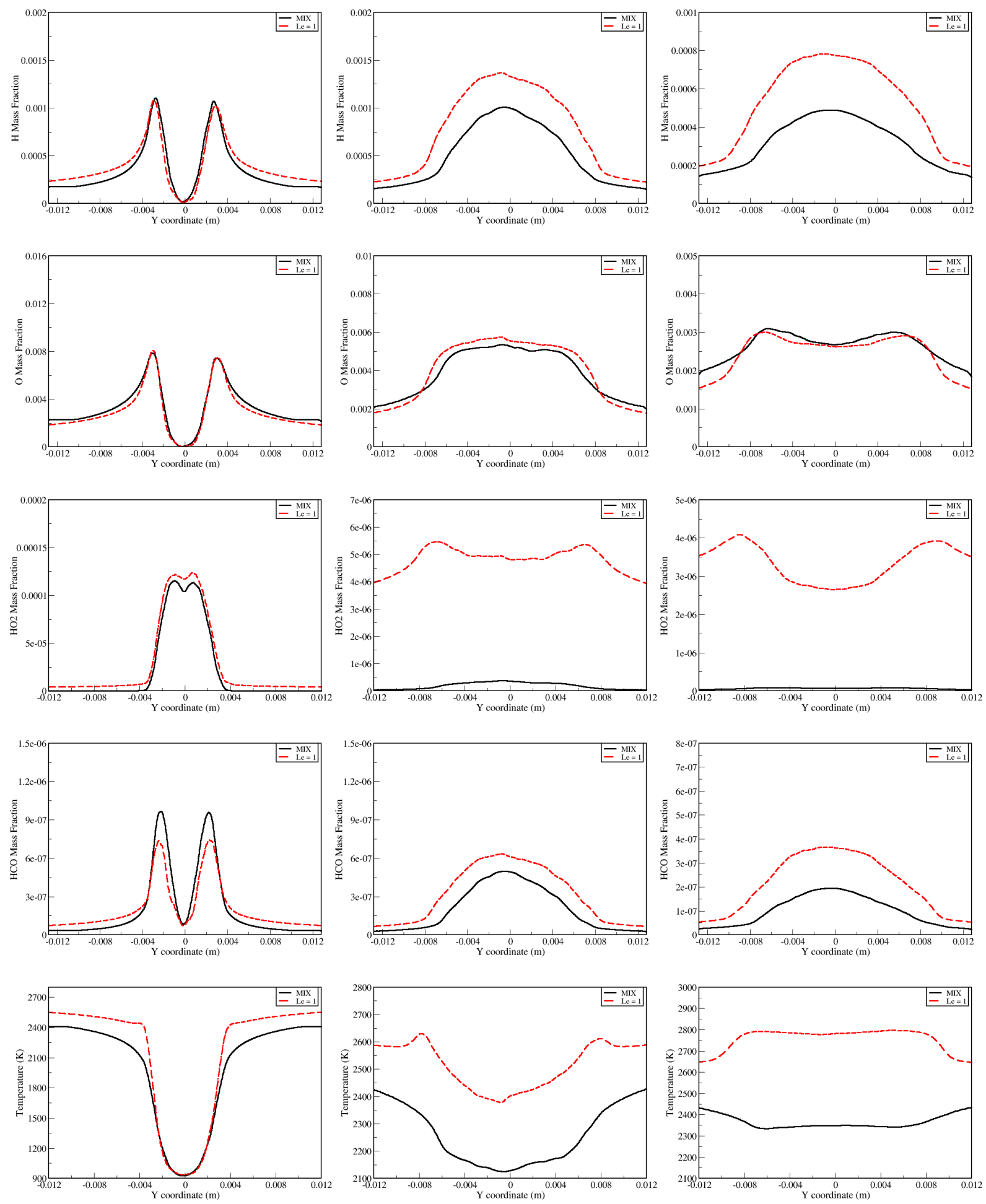

Figure 15: $\mathrm{H}, \mathrm{O}, \mathrm{HO}_{2}, \mathrm{HCO}$ and temperature profiles predicted by LESLIE with mixtureaveraged diffusion and with constant Lewis number $=1$. Left to right: $t^{\star}=2 / 3,4 / 3$ and 2 . Black, MAD; red, Le=1 model. 
Note that Le $=$ constant means that heat and mass diffusivities stay in the same ratio no matter what, and are exactly equal for all species if $L e_{i}=1$; that does not mean that heat and mass fluxes are equal. However, the results shown in figs. 14 and 15 imply that, generally speaking, the $L e=1$ model produces more diffusive fields than MAD or MCD, with differences depending on the specific species transported. Faster diffusion with $\mathrm{Le}=1$ results in equivalence ratio closer to unity and justifies temperatures $\sim 200$ to $350 \mathrm{~K}$ higher than predicted with MAD. These temperature differences are unexpectedly large. Were they to be found at the scales typical of some high intensity burners and combustors $(\sim 10 \mathrm{~cm}$ for aero-engines), i.e., were they to statistically persist in space and time, the conclusion would be that cheaper and simpler diffusion models cannot predict realistic turbulent flow fields. This finding would be even more intriguing if diffusion effects were also found at higher pressure. Aero-engine combustors are now operating at pressure ranging from 40 to 50 bars at take-off and to a slightly lower 10-20 bars at cruise. Thus Reynolds numbers should be one to two orders of magnitude larger than simulated in this work. Gradients should be much sharper and pressure driven diffusion significant, but diffusivities lower; without repeating DNS at Re numbers at least a factor of ten higher, it is difficult to predict the effect of these competing mechanisms. In any event, consequences on NOx, soot, particulate formation and hot corrosion should be very interesting.

Missing from this sequence of simulation is a DNS using different $L e_{i}$ for each $i^{\text {th }}$ species, for instance using the temperature-averaged $L e_{i}$ data from LES of a reacting $\mathrm{H}_{2} / \mathrm{CH}_{4}$ /air mixture in [15]. However, its significance is uncertain, because $L_{i}$ extracted from a specific LES are not universally valid. Alternatively, averaged $L e_{i}$ could be extracted from a laminar premixed flame, although with similar caveat.

\section{Discussion}

The most interesting result of this investigation is that for sufficiently high Reynolds numbers molecular diffusivity is significant, just as Barlow et al at Sandia concluded from their experiments. In fact, evidence from the present work adds preferential diffusion to convective transport and kinetics as the third important mechanism present in turbulent combustion. If confirmed by further work, common understanding that turbulence transports equally all species will have to be revised. A second indirect effect noted in [17] is that lighter species such as $\mathrm{OH}$, $\mathrm{H}_{2} \mathrm{O}$ and $\mathrm{H}$ diffuse faster out of the reaction zone, leaving behind (so to speak) heavier and slower species such as $\mathrm{CO}_{2}$.

The observed impact of molecular diffusion is due to two factors. The first is that diffusion transport scales with the magnitude of diffusion coefficients and with gradients (in species, temperature and pressure): simply comparing molecular and eddy diffusivities independently of the Reynolds number is misleading. Scalar gradients increase as the inverse of the turbulent scale; if the Oboukhov-Corrsin spectrum is valid, molecular mixing takes place at scales not too far from Kolmogorov"s [30], and gradients become more than capable of making up for the difference between molecular and eddy diffusion coefficients. At scales $\ell$ close to dissipative, diffusion time scales as $\ell^{2} /\left[D_{i}\left(R e_{\ell}\right)^{3 / 2}\right]$, while eddy time as $\ell^{2} /\left(v R e_{\ell}\right)$ : diffusion may become faster than turbulent transport when $\mathrm{Re}_{\ell}$ becomes large. Sharpening of gradients with increasing Reynolds number is faster for molecular than for eddy diffusion and is opposed by the effect of 
heat release smoothing gradients. This effect is tempered by transport properties increasing with temperature [31]; it may be reinforced, or attenuated, by the baroclinic effect. (Had the vorticity $(\boldsymbol{\omega})$ rather than the momentum equation been solved, these considerations would be better quantified by analyzing the order of magnitude of the $\boldsymbol{\omega}$ equation terms. However, solving for $\boldsymbol{\omega}$ poses problems, because all scalar equations contain velocity that must be extracted from $\boldsymbol{\omega}$, and also because such alternative approach is not implemented in any CFD code, and would have to be developed from scratch.)

The second factor is linked to faster diffusion by lighter radical species. In hydrocarbon/air combustion these are mostly $\mathrm{H}$ and $\mathrm{OH}$ radicals, possessing positive standard enthalpies of formation. At $2000 \mathrm{~K} \mathrm{OH}$ has $\Delta \mathrm{H}_{\mathrm{f}}{ }^{\circ}=\sim 37 \mathrm{~kJ} / \mathrm{mol}$; $\mathrm{H}$ has $\sim 220 \mathrm{~kJ} / \mathrm{mol}$ : even in small concentrations, their preferential transport may significantly affect pathways, reaction rates and, probably, the temperature differences shown in fig.15. Moreover in hydrocarbon flames, hydrogen is produced in the reaction zone, and hence, may exhibit even stronger diffusion effects locally in the flame zone where it can affect species distributions, reaction rates and reaction pathways. Thus local features of turbulent reacting fields should not be interpreted solely in terms of kinetics and turbulence, since molecular transport may interfere selectively with the ,species-blind"convective transport.

Taking these DNS results at face value, it is tempting to conclude that LES of aero-engine combustors with simple diffusion models (constant Lewis numbers, Fick"s law) routinely over predict local temperatures. Such conclusion is premature: the results presented are from DNS, not LES, and might be valid only in this specific test case. This said, it still seems possible to conclude that the constant Le model tends to produce unphysical diffusion, either faster or slower than with MCD depending on the particular species and conditions, therefore depending on fuel and equivalence ratio. Unphysical diffusion is certainly responsible for the over prediction of temperature in fig.15, with significant impact on emissions. Preferential diffusion of fuel species has already been shown to affect flame extinction predictions [32].

Even implementing higher fidelity diffusion models (like MAD or MCD) in LES would not improve simulation realism unless molecular transport at subgrid scales was also modeled. It is typical to neglect subgrid molecular fluxes in most LES and model only subgrid turbulent fluxes. Thus subgrid turbulence-chemistry-diffusion interactions may be incorrect. One way to assess the impact of diffusion in LES consists of explicitly filtering DNS data at a number of scales and of calculating the ratio between diffusion flux and SGS (turbulent flux). At this stage this would help to clarify the detailed sequence of cause and effect producing the results discussed. One such study is reported for non-premixed Hydrogen-air and Methane-air jet flames in [33]. More resources and DNS of different test cases are necessary, also to verify whether diffusion effects are found in non-premixed flames, far more common in applications.

These considerations apply at the scale of this test case; the residence time in actual combustors is longer than the fraction of millisecond simulated here: large scale mixing might „wash out" the effect of diffusion. Whether this occurs should be investigated by performing LES of realistic geometries with the MAD or MCD model. The prerequisite is the development of 
SGS molecular diffusion closures for species (and energy) equations. Hopefully, this will be possible in the future.

\section{Conclusions}

Within the assumptions made, and for the test case simulated in this work, it is possible to conclude that at high Reynolds numbers turbulent combustion predictions depend on how accurately molecular diffusion is modeled, and thus molecular diffusion cannot be neglected. In fact, it may be the third important mechanism influencing species formation and energy release besides turbulence and chemical kinetics.

Comparison of DNS results obtained with the three diffusion models tested shows that either the MCD or MAD model predicts essentially the same fluid-dynamic and thermo-chemical field. However, the constant $\mathrm{Le}_{i}$ model predicts a significantly different flow-field. Thus physically realistic diffusion must be implemented to capture its physics (species and especially temperature) correctly.

Molecular diffusion is the last step in transporting reactive species to and inside turbulent flames; it is due to collisions, the same physical mechanism responsible for kinetics, so a posteriori the conclusion above may appear obvious. If the impact of diffusion is confirmed at higher pressures and at scales much larger than in this DNS, replacing simpler diffusion models with MAD/MCD models in LES will improve understanding of turbulent combustion and produce more realistic simulations of industrial applications. Development of SGS molecular diffusion closures for species and energy equations should be the next step in this direction.

\section{$\underline{\text { Acknowledgements }}$}

The authors wish to thank Dr. Catalin Fotache and Dr. Joe Sangiovanni for supporting this work internally and for useful technical discussions. The authors also wish to thank Dr. Jeremiah Lee for many in-depth discussions on the subject of SGS models of multi-component diffusion. Special thanks are due to Dr. Robert Barlow at Sandia National Laboratories, Livermore, CA for information and clarification of experimental data found by his team. 


\section{Appendix A: Molecular Diffusion Models}

Like chemical kinetics, molecular diffusion is the result of intermolecular collisions, Modeling diffusion can therefore be derived either from statistical kinetic theory or from considerations tied to the Second Principle and thus to non-equilibrium thermodynamics.

The kinetic theory approach [34] [35] is based on expanding the collision integral on the right hand side of the Boltzmann equation, and solving it to $1^{\text {st }}$ order with the help of the mathematical expression for the diffusion vectors $\mathbf{d}_{\mathbf{i}}$ in an ideal gas [36]. Non-equilibrium thermodynamics [37] is more general, as it can be applied to real gases and liquids; the motion of molecular species is assumed driven by the thermodynamic activity function. The $1^{\text {st }}$ order expression for the $\mathbf{d}_{i}$ in [34] was also obtained in [37].

In the kinetic approach the statistical motion of individual species in a mixture is calculated as the result of all possible bimolecular collisions among all species pairs. (Tri-molecular collisions become important at supercritical pressure and in liquids.) This formulation leads to models of diffusion transport called preferential, poli-nary, or multi-component, MCD for short. Although less general than that using thermodynamics, the kinetics approach does not require calculating the thermo-chemical activity of each species, blends more easily with the Navier-Stokes equations and has been chosen here.

Using the kinetic approach, and omitting contributions due to body forces, the diffusion velocity $\boldsymbol{V}_{\boldsymbol{i}}$ may be obtained by following one of two routes. The first calculates each $\boldsymbol{V}_{\boldsymbol{i}}$ as the sum of three contributions, due to gradients of concentration, pressure, and temperature (the Soret effect); each can be calculated separately for all ns species, see equation (1).

$$
X_{i} \boldsymbol{V}_{i}=-\sum_{j}^{n s} D_{i j}\left[\nabla X_{j}+\left(X_{j}-Y_{j}\right) \nabla \ln (p)\right]-D_{T i} \nabla \ln (T)
$$

Equation (1) requires calculating the coefficients, $D_{\mathrm{ij}}$ and $\mathrm{D}_{\mathrm{Ti}}$, that depend on the specific choice of intermolecular potential and on the kinetic energies of each molecule pair. Equation (1) is found by expanding to $1^{\text {st }}$ order the Sonine polynomials series representing the three contributions to the distribution function $\mathrm{f}\left(\mathbf{X}, \boldsymbol{V}_{\boldsymbol{i}}\right)$ in the Boltzmann equation [34]. To $1^{\text {st }}$ order, the coefficients are reported in [28] [38] as functions of $T, p$ and local mixture composition. The $D_{i j}$ coefficients are the same for the pressure and concentration terms and do not involve ratios of determinants, as does instead calculating $\mathrm{D}_{\mathrm{T}}$, a $2^{\text {nd }}$ order term in the expansion.

Inspection of the mathematical expressions for $D_{i j}$ and $D_{T i}$ shows that both, especially $D_{T i}$, are computationally expensive. Provided that all input data for the species present are available, the EGLib [39], CANTERA [40] and CHEMKIN [38](Transport) suites of subroutines are the standard tools to perform efficiently these calculations. Even so, the computational burden of evaluating $\mathrm{D}_{\mathrm{Ti}}$ remains significant.

The second route results in an implicit expression for $\boldsymbol{V}_{i}$, [21]. This expression links the gradient of volume fraction of each species to the sum of differences between each pair of diffusion velocities over all ns species, plus the Soret and pressure diffusion terms. The coefficients appearing in this expression, see equation (2), are binary diffusion coefficients, $\mathcal{D}_{i j}$, associated 
to collisions in individual binary mixtures and depend only on $\mathrm{T} . \mathcal{D}_{i j}$ for many species pairs have been calculated, tabulated and fitted in the particular case of the Lennard-Jones 12-6 potential [28]. This simple potential is convenient when calculating collision integral, although it becomes increasingly inaccurate with increasing $T$ [41] [42]. A key disadvantage of this route is that each $\mathbf{V}_{\mathbf{i}}$ cannot be obtained as a sum of separate contributions, but only by inverting the implicit equation (3) for $\mathbf{V}_{\mathbf{i}}$ :

$$
\nabla X_{i}=\sum_{j}^{n s}\left(\frac{X_{i} X_{j}}{D_{i j}}\right)\left(\boldsymbol{V}_{j}-\boldsymbol{V}_{i}\right)+\left(Y_{i}-X_{i}\right) \nabla \ln (p)+\sum_{j}^{n s}\left(\frac{X_{i} X_{j}}{\rho \mathcal{D}_{i j}}\right)\left(\frac{D_{T j}}{Y_{j}}-\frac{D_{T i}}{Y_{i}}\right) \nabla \ln (T)
$$

A model simpler than either of the multi-component models outlined was developed by Hirschfelder and coworkers [22]; it assumes molecular species diffuse by ,colliding" with the local mixture (Mixture-Averaged Diffusion, or MAD model). Their coefficients, $D_{\mathrm{im}}$, are semiempirical, see for instance [15] [42] [43].

\section{References:}

[1] Y. B. G. L. V. a. M. G. Zel"dovich, The Mathematical Theory of Combustion and Explosions, New York: Plenum Press, 1985, p. Chapter 1 and 5.

[2] W. Meier, A. O. Vyrodov, V. Bergmann and W. Stricker, Appl. Phys. B 63, pp. 79-90, 1996.

[3] W. Meier, S. Prucker, M. H. Cao and W. Stricker, Comb. Sci. Tech. 118, pp. 293-312, 1996.

[4] V. Bergmann, W. Meier, D. Wolff and W. Stricker, Appl. Phys. B 66, pp. 489-502, 1998.

[5] A. Kronenberg and R. W. Bilger, Phys. Fluids 9, pp. 1435-1442, 1997.

[6] R. S. Barlow and J. H. Frank, in Poster W2E07, Third International Workshop on Measurements and Computation of Turbulent Nonpremixed Flames, Boulder, 1998.

[7] R. S. Barlow, M. J. Dunn, M. S. Sweeney and S. Hoechgreb, Combust. Flame, 159, pp. 2563-2575, 2012.

[8] M. S. Sweeney, S. Hochgreb, M. J. Dunn and R. S. Barlow, Combust. Flame 159, pp. 2886-2911, 2012.

[9] M. S. Sweeney, S. Hochgreb, M. J. Dunn and R. S. Barlow, Combust. Flame 159 (2012), pp. 2912-2929, 2012.

[10] R. Hilbert, F. Tap, H. El-Rabii and D. Thevenin, Prog. Ener. Combust. Sci. 30, pp. 61-117, 2004.

[11] P. K. Yeung and S. B. Pope, Phys. Fluids A 5, p. 2467, 1993. 
[12] V. Nielsen and G. Kosaly, Phys. Fluids 9 , p. 3386, 1997.

[13] V. Nielsen and G. Kosaly, Combust. Flame 117, pp. 493-513, 1999.

[14] H. Pitsch, Combust. Flame 123, pp. 358-374, 2000.

[15] E. Giacomazzi, F. R. Picchia and N. Arcidiacono, Combust. Theory and Modelling 12, pp. 135-142, 2008.

[16] F. Dietzsch, G. Fru, D. Thevenin and C. Hassa, in Proc. ERCOFTAC Direct and LargeEddy Simulation IX, Dresden, April 3-5, 2013.

[17] M. J. Dunn and R. S. Barlow, Proc. Comb. Inst. 34, pp. 1411-1419, 2013.

[18] R. S. Barlow, M. J. Dunn and G. Magnotti, Combustion and Flame, vol. 162, pp. 727-735, 2015.

[19] V. R. Katta and W. M. Roquemore, Proc. Combust. Inst. 34, pp. 1101-1108, 2013.

[20] L. Vervisch and S. Hochgreb, in Proc. 11th Int. Workshop on Measurements and Computation of Turbulent (Nonpremix) Flames, Darmstadt, Germany, 2012.

[21] F. A. Williams, Combustion Theory, The Benjamin Cummings Publisher Co., 1985.

[22] E. R. Hawkes, R. Sankaran, J. C. Sutherland and J. H. Chen, Combust. Flame 31, pp. 1633-1640., 2007.

[23] J. Li, "PhD Thesis 3122-T, Mech. and Aerospace Dept.," Princeton University, Princeton, New Jersey, 2004.

[24] S. B. Pope, Turbulent Flows, Cambridge University Press, 2000.

[25] T. J. Poinsot and S. K. Lele, J. Comp. Phys. 101, pp. 104-129, 1992.

[26] S. Menon, "LESLIE - LES solver from Georgia Tech," http://www.ccl.gatech.edu, 2015.

[27] J. Chen, "S3D - DNS solver from Sandia Labs," 2015.

[28] R. J. Kee, M. E. Coltrin and P. Glarborg, Chemically Reacting Flows: Theory and Practice (Chapter 12), New York: J. Wiley, 2003.

[29] V. R. Katta, L. Goss and W. M. Roquemore, Combust. Flame 96, pp. 60-69, 1994.

[30] K. R. Sreeenivasan, Phys. Fluids 8 , pp. 189-196., 1996. 
[31] K. C. Bray, P. A. Libby, G. Masuya and J. B. Moss, Comb. Sci. Tech. 25 , pp. 127-140, 1981.

[32] S. H. Won, S. Dooley, F. L. Dryer and Y. Ju, "A radical index for the determination of the chemical kinetic contribution to diffusion flame extinction of large hydrocarbon fuels.," Combustion and Flame, vol. 159, no. 2, pp. 541-551, 2012.

[33] J. C. Sutherland, P. J. Smith and J. H. Chen, "Quantification of differential diffusion in nonpremixed systems," Combustion Theory and Modeling, vol. 9, no. 2, pp. 365-383, 2005.

[34] J. O. Hirschfelder, C. F. Curtis and R. B. Bird, in The Molecular Theory of Gases and Liquids, New York, John Wiley, 1954, p. Section 7.3 to 7.4.

[35] R. B. Bird, W. E. Stewart and E. N. Lightfoot, Transport Phenomena (Chapter 18), New York: John Wiley, 2002.

[36] S. Chapman and T. G. Cowling, The Mathematical Theory of Non-Uniform Gases. Ch. 18., Cambridge: Cambridge University Press, 1990.

[37] H. J. Merk, Appl. Sci. Res. A 8, pp. 73-89, 1958.

[38] R. J. Kee, G. Dixon-Lewis, J. Warnatz, M. E. Coltrin, J. A. Miller and H. K. Moffat, "Sandia Report SAND86-8246 B \& Reaction Design Report TRA-036-1 (2000).," Livermore, CA, 1986.

[39] A. Ern and V. Giovangigli, "EGLib: A General Purpose Fortran Library For Multicomponent Property Evaluation, EGLib Manual, version 3.4," 2004.

[40] "CANTERA," http://cantera.github.com/docs/sphinx/html/index.html, 2014.

[41] P. Kirch and R. Schutte, Chem. Phys. 42, pp. 3729-3730, 1965.

[42] T. Poinsot and D. Veynante, Theoretical and Numerical Combustion, Chapter 1., Bordeau, France: Aquaprint, 2013.

[43] G. C. Maitland, M. Rigby, E. B. Smith and W. A. Wakeham, Intermolecular Forces, Chapter 9., Oxford, UK: Clarendon Press, 1981. 
Figure - 1b
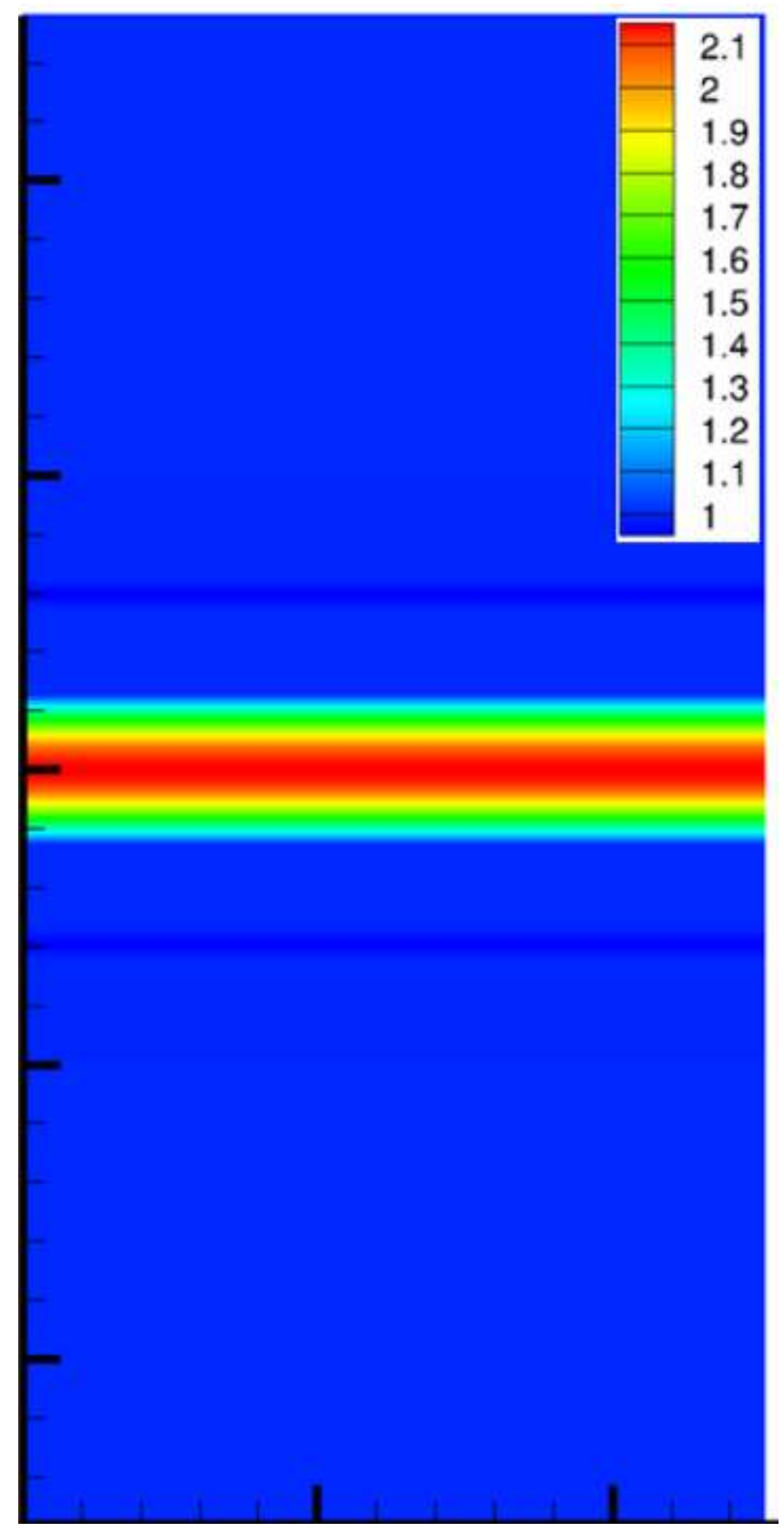
Figure - 1c

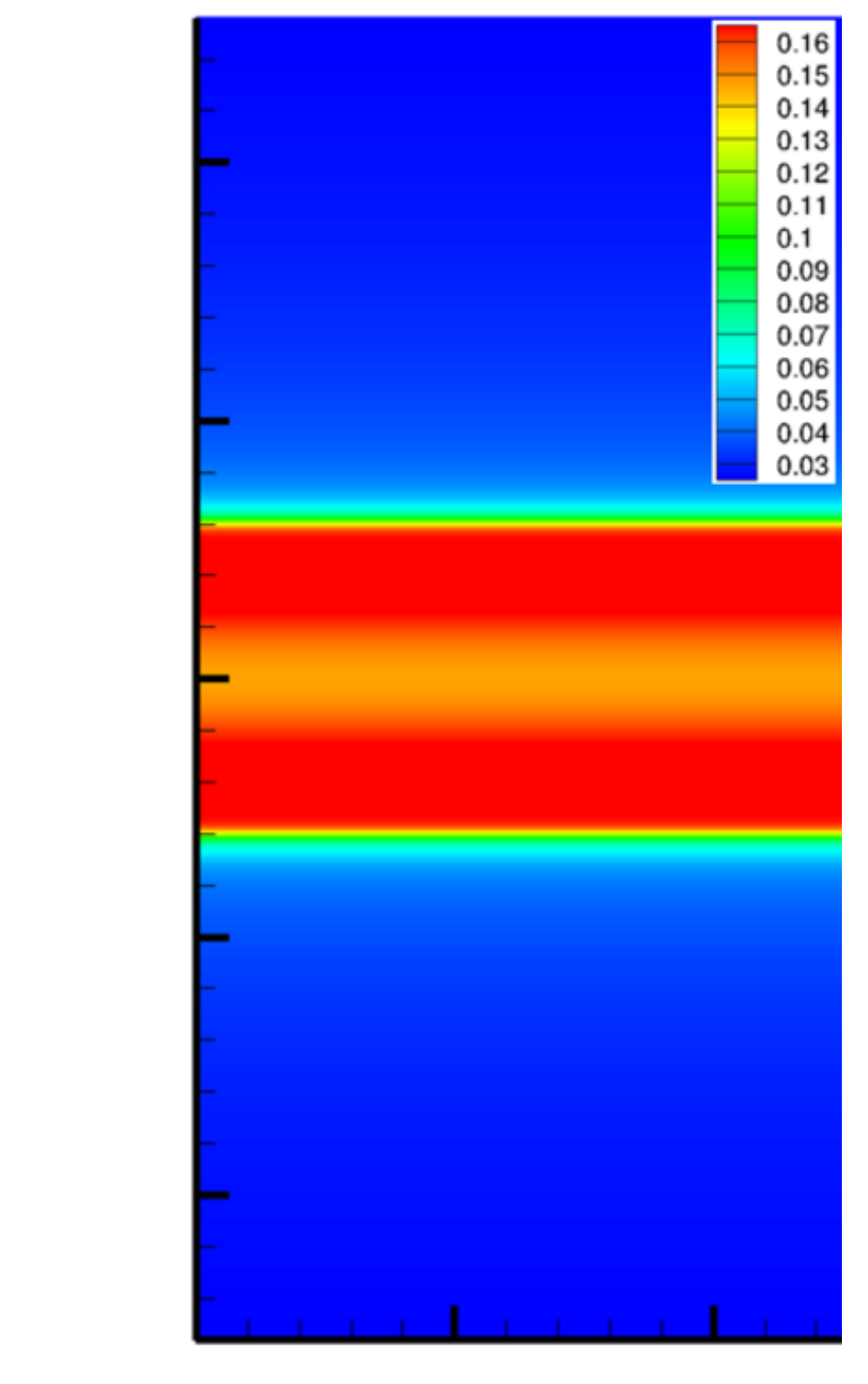

(c)

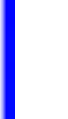

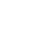

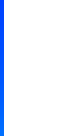

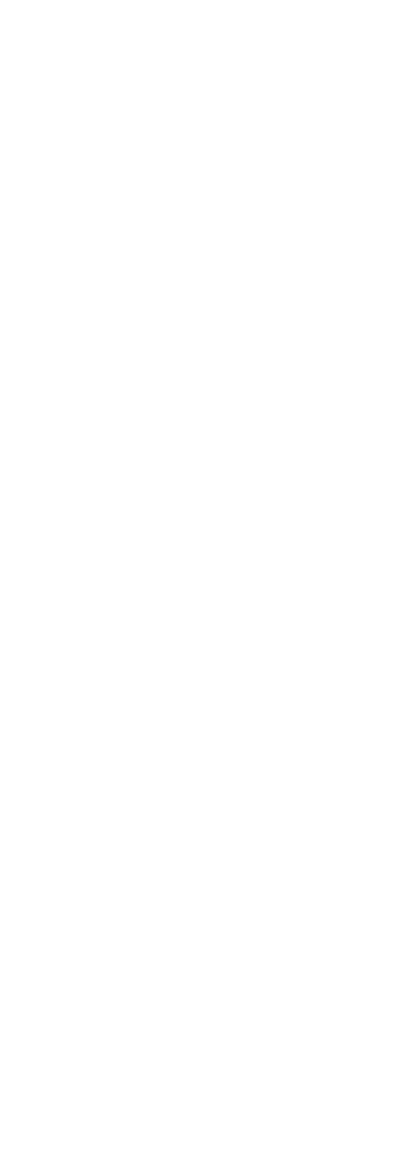


T

2.5E + 03

$9.0 E+02$

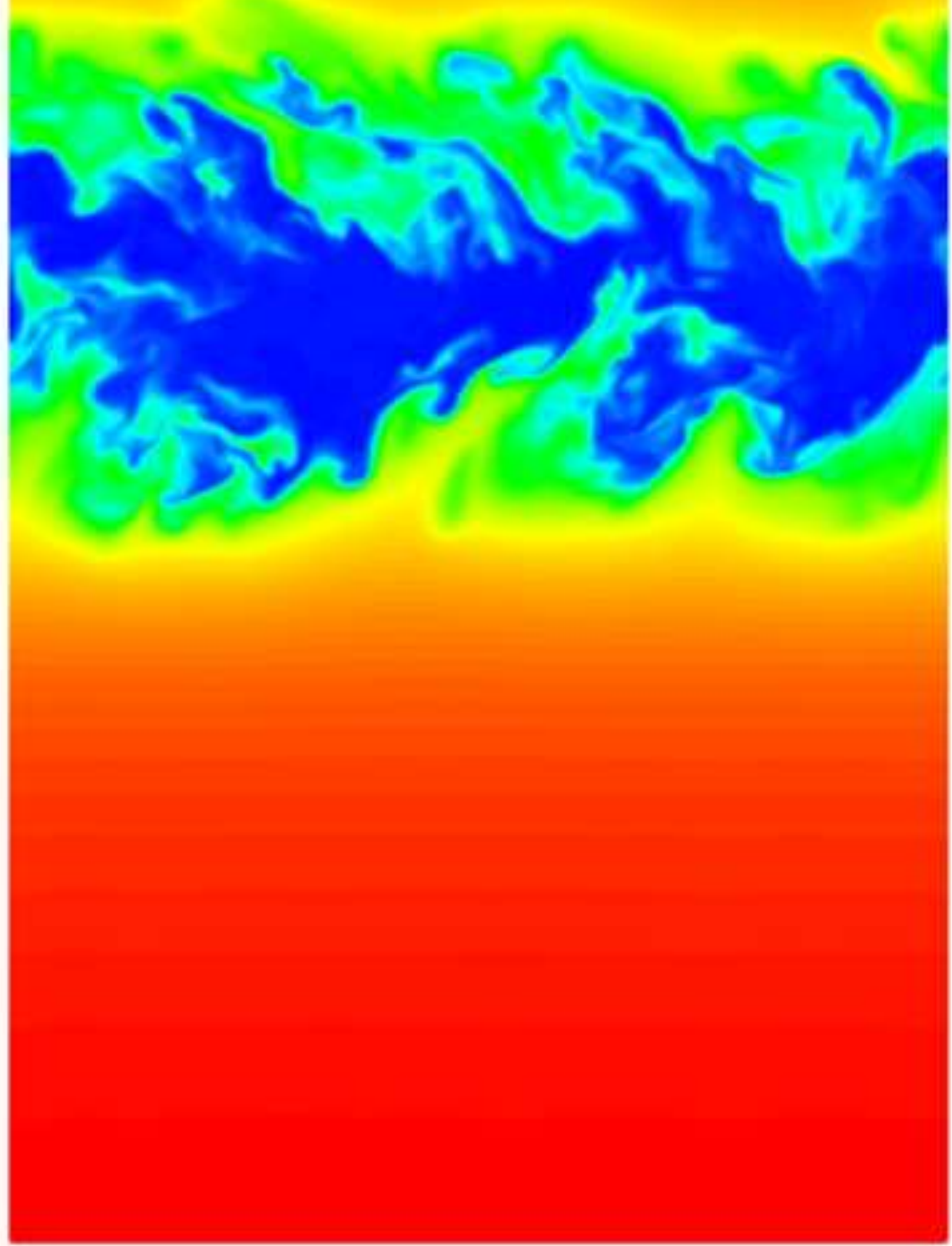




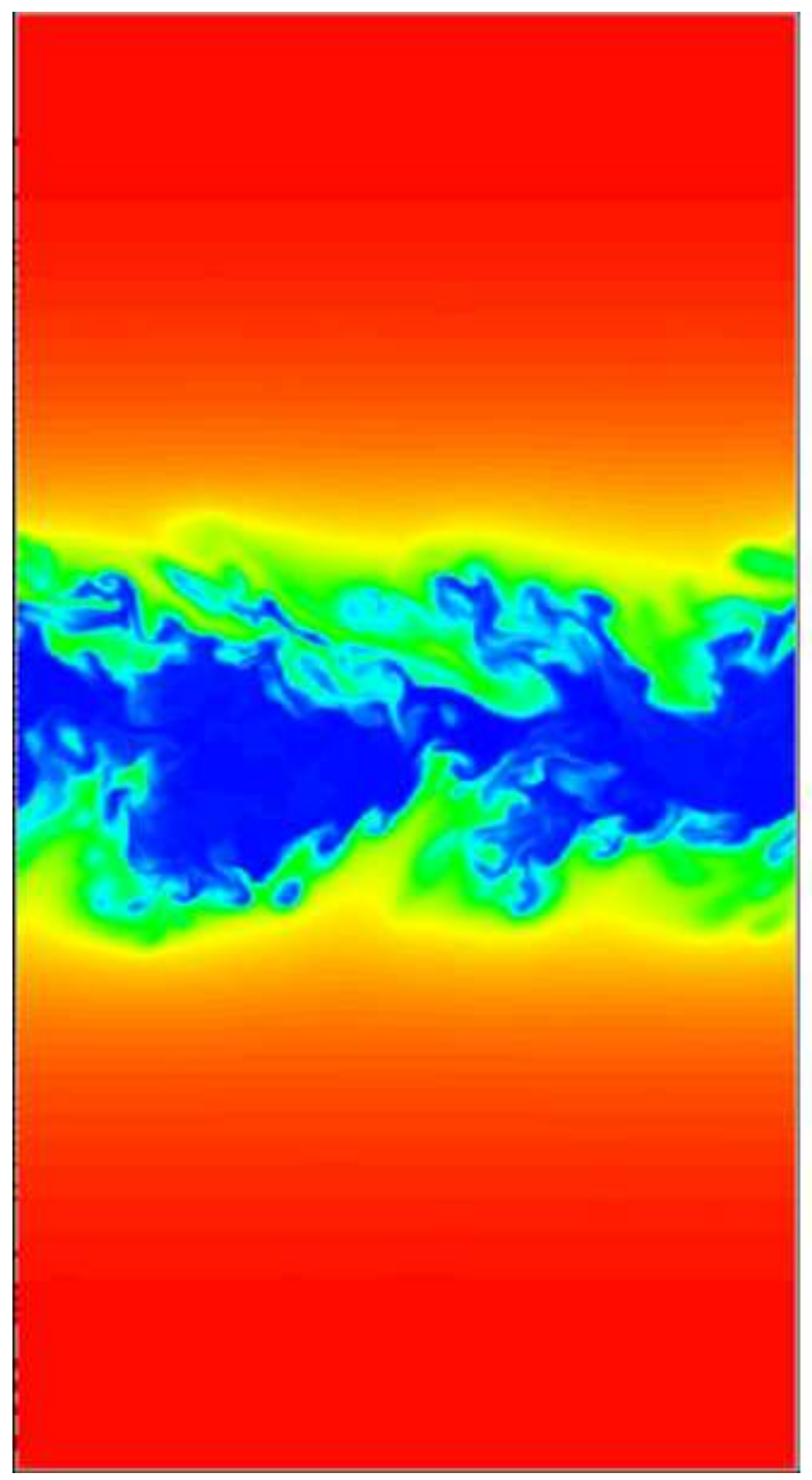

$\mathrm{T}(\mathrm{K})$

2400 2300 2200 2100 2000 1900 1800 1700 1600 1500 1400 1300 1200 1100 1000

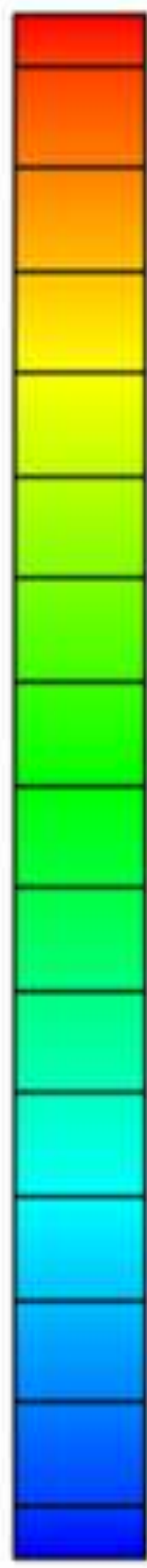

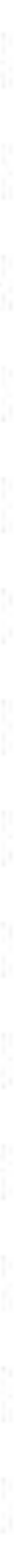


Figure - 4a

T

2.5E + 03

$9.0 E+02$

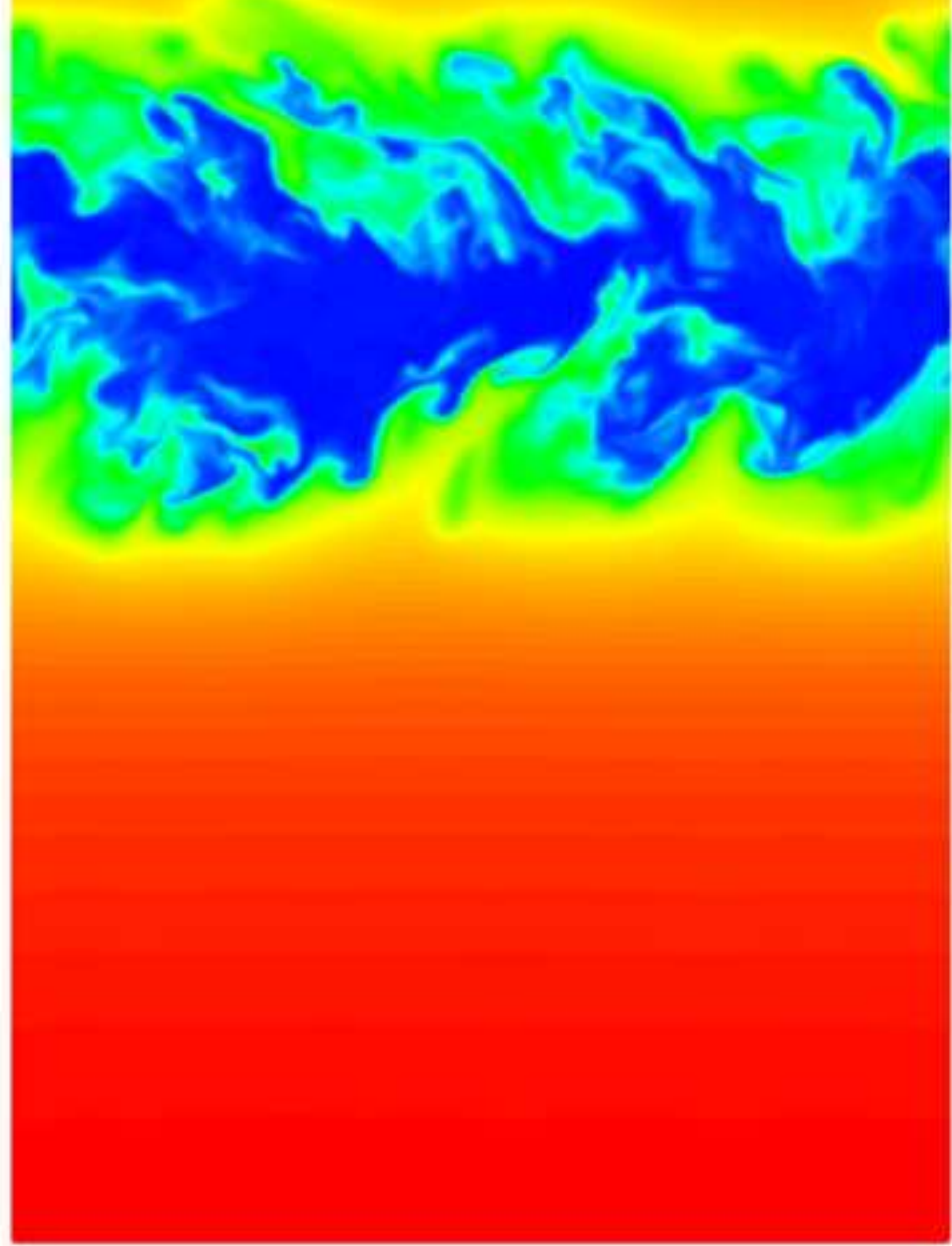




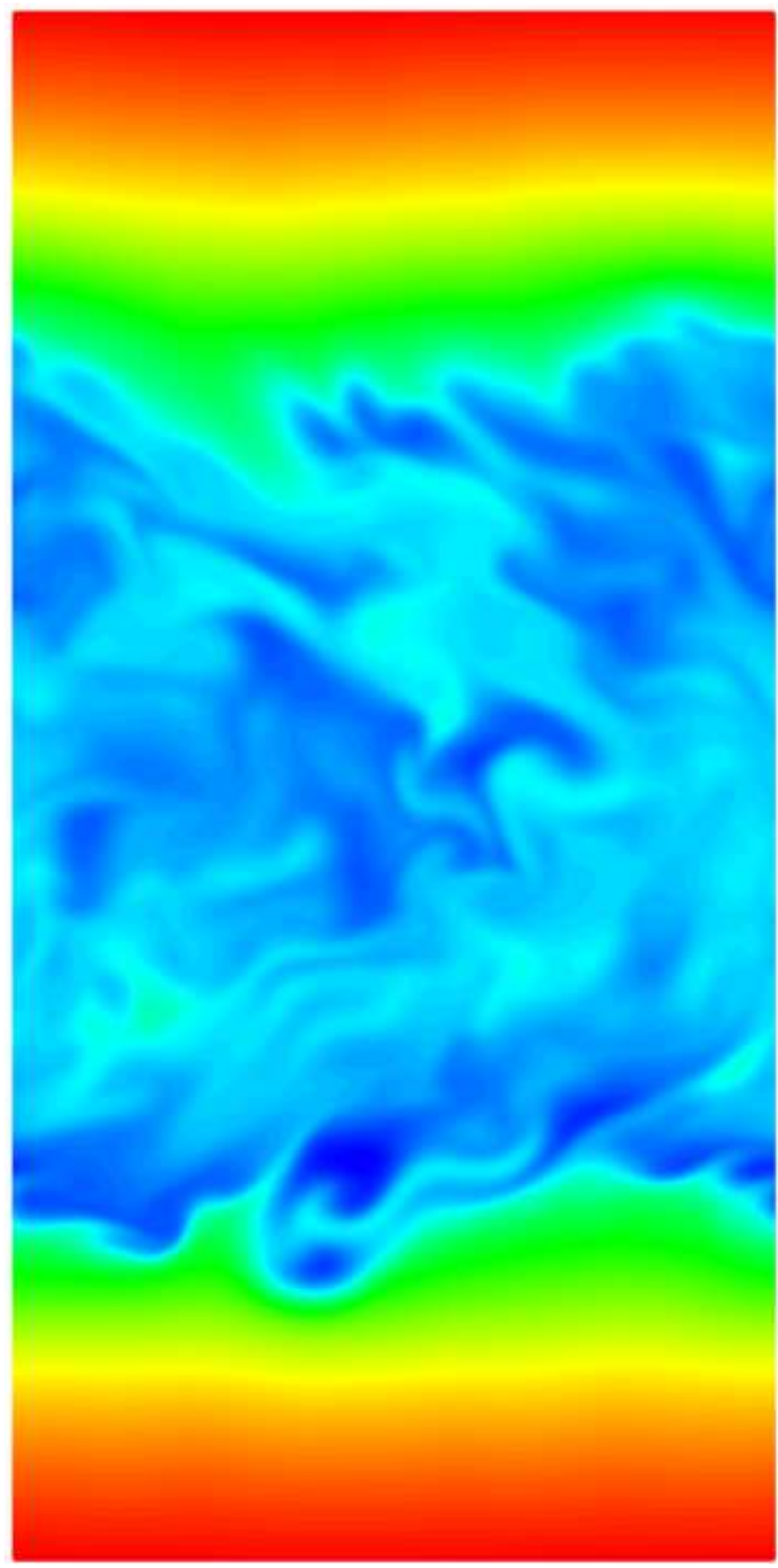

2406.3

\section{6}

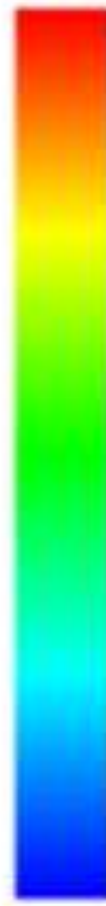




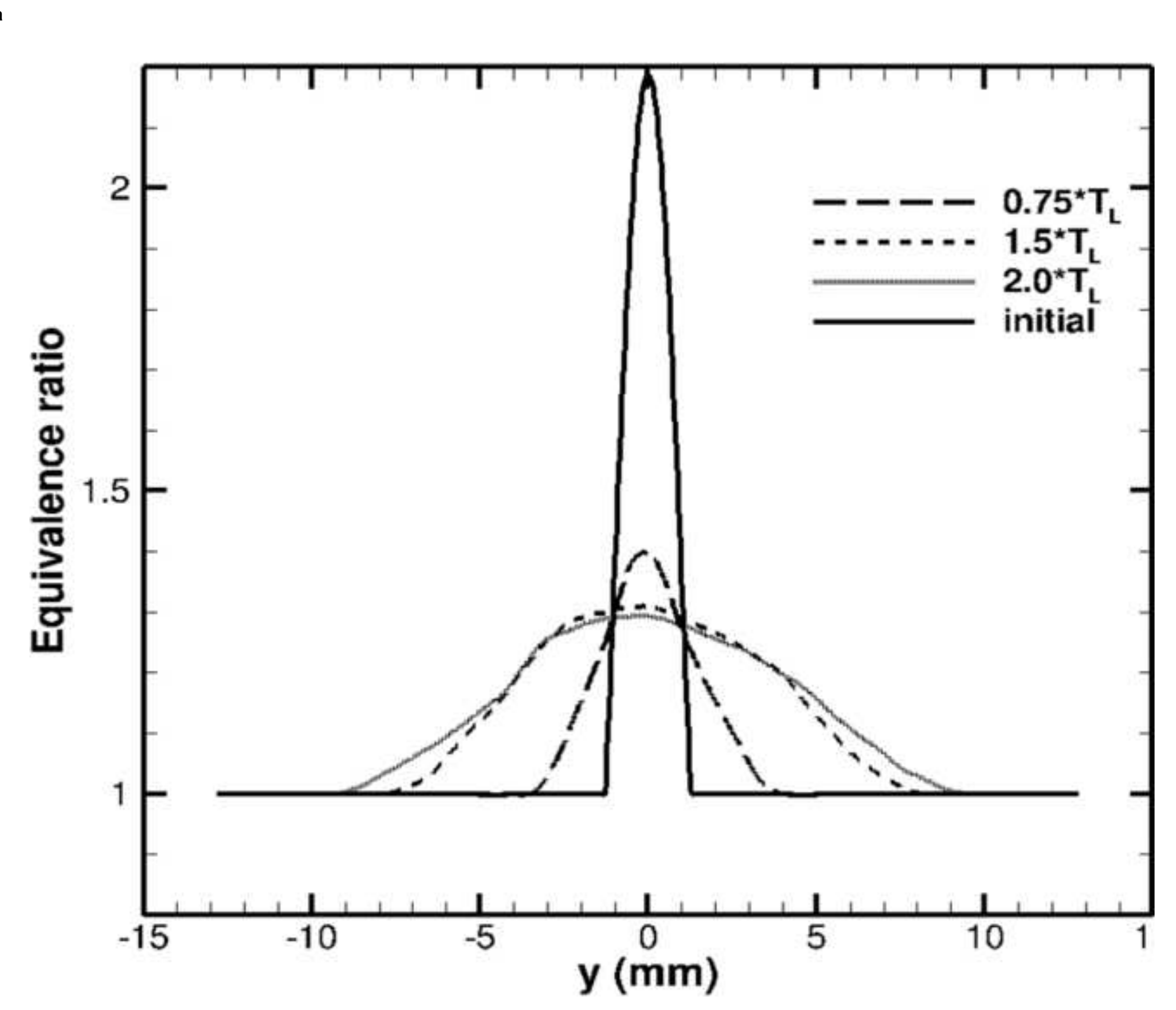

Fure - $5 a$

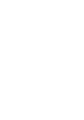




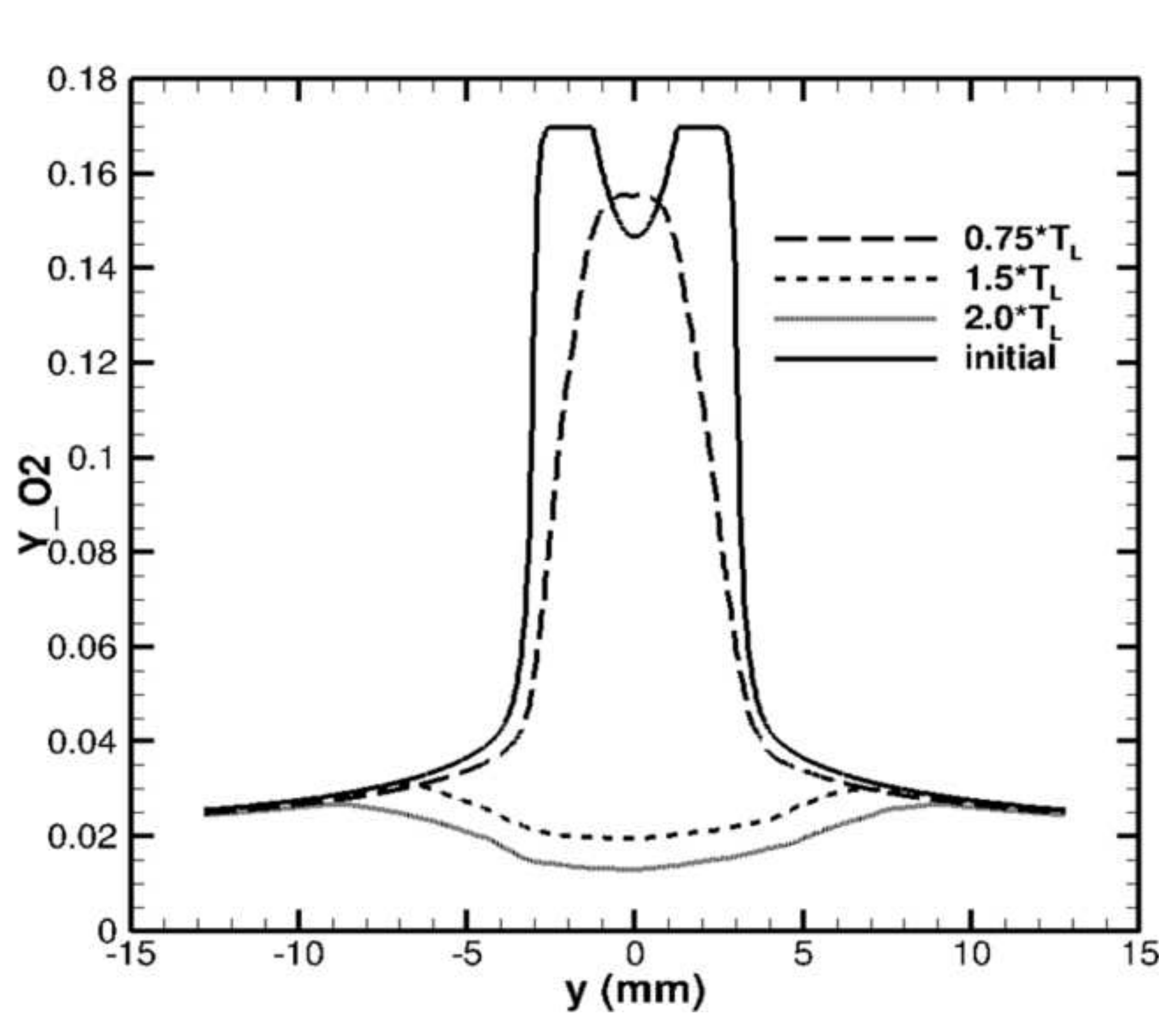




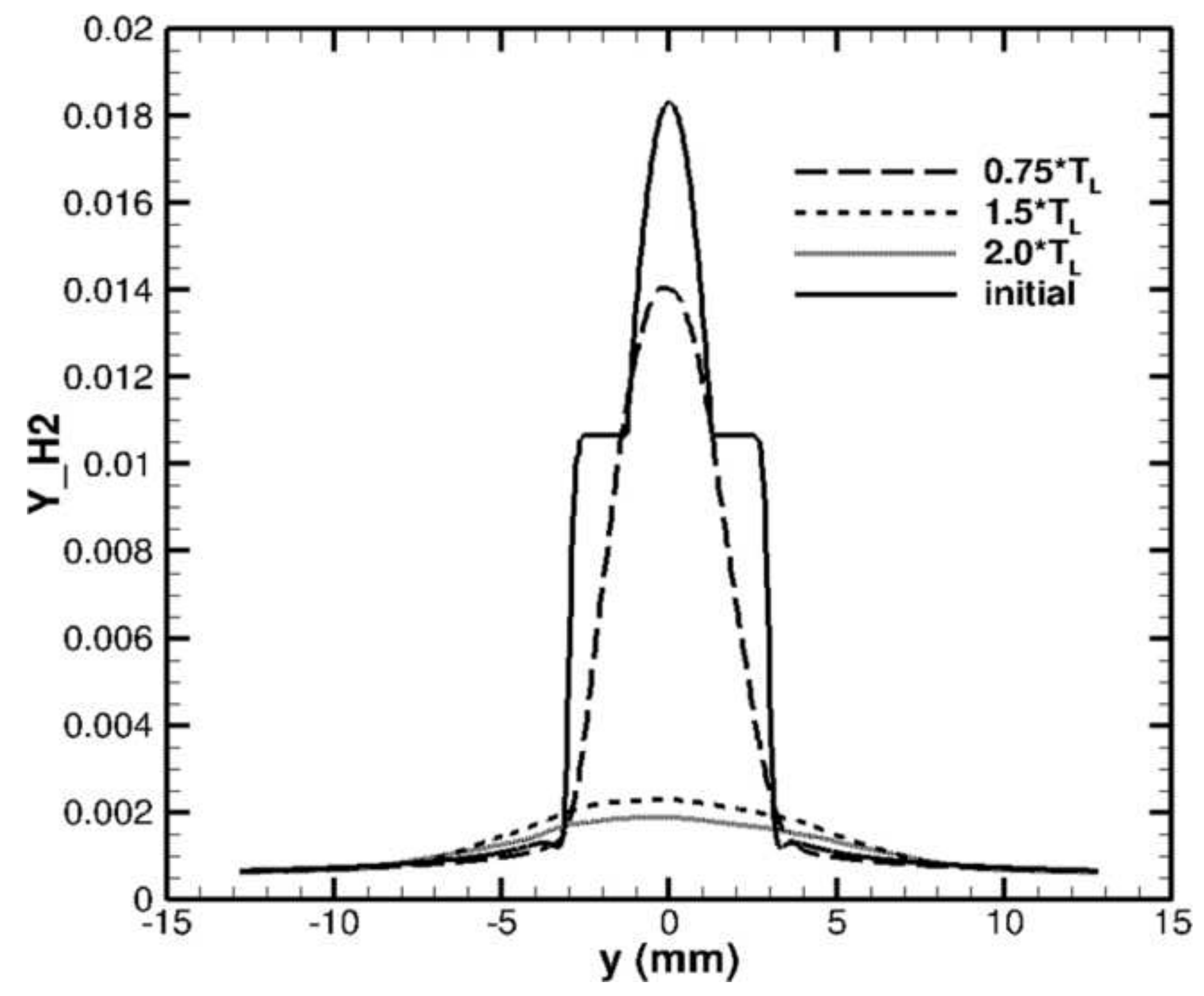




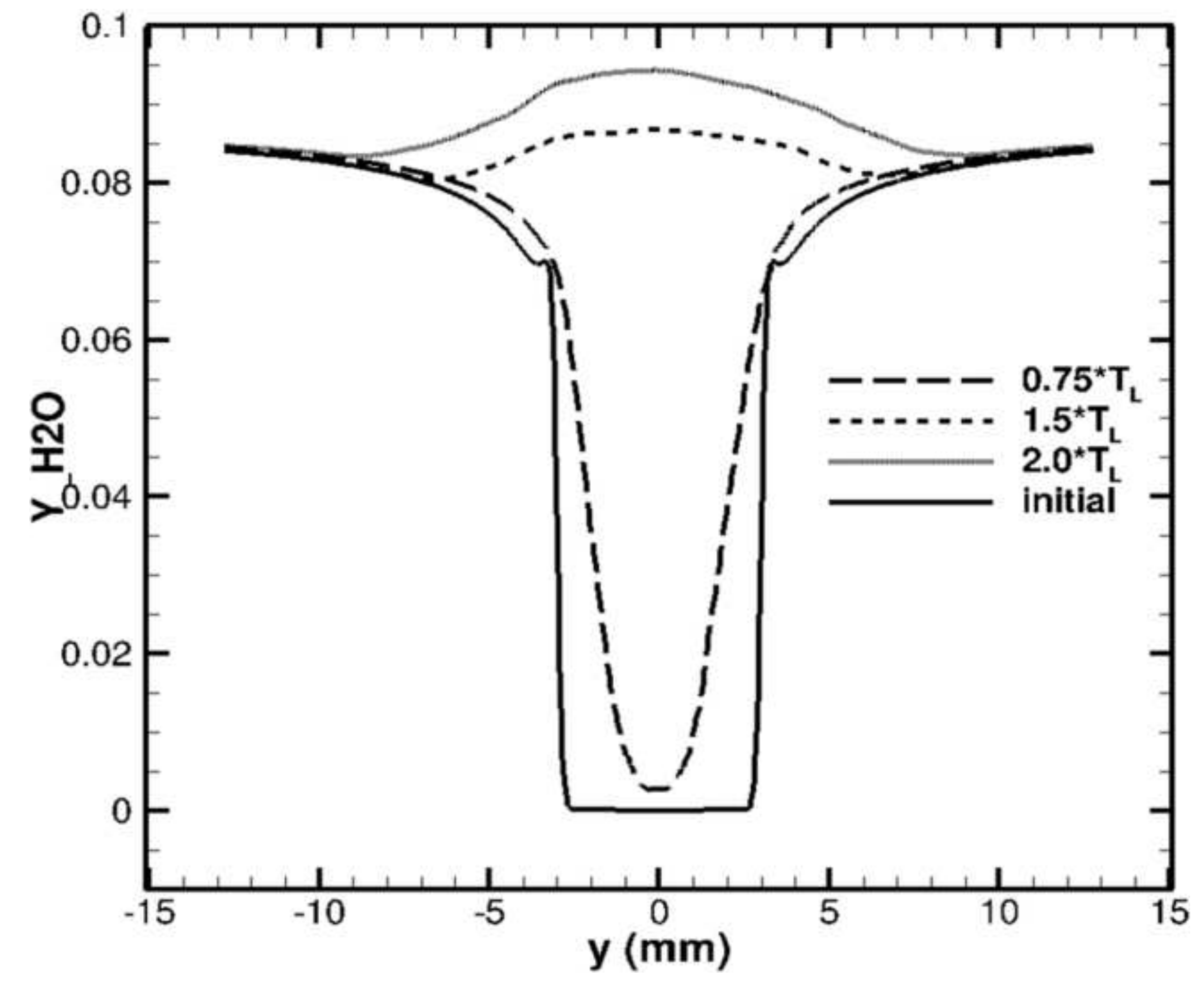


Figure - $6 a$

CO

\subsection{E-01}

3.5E-02

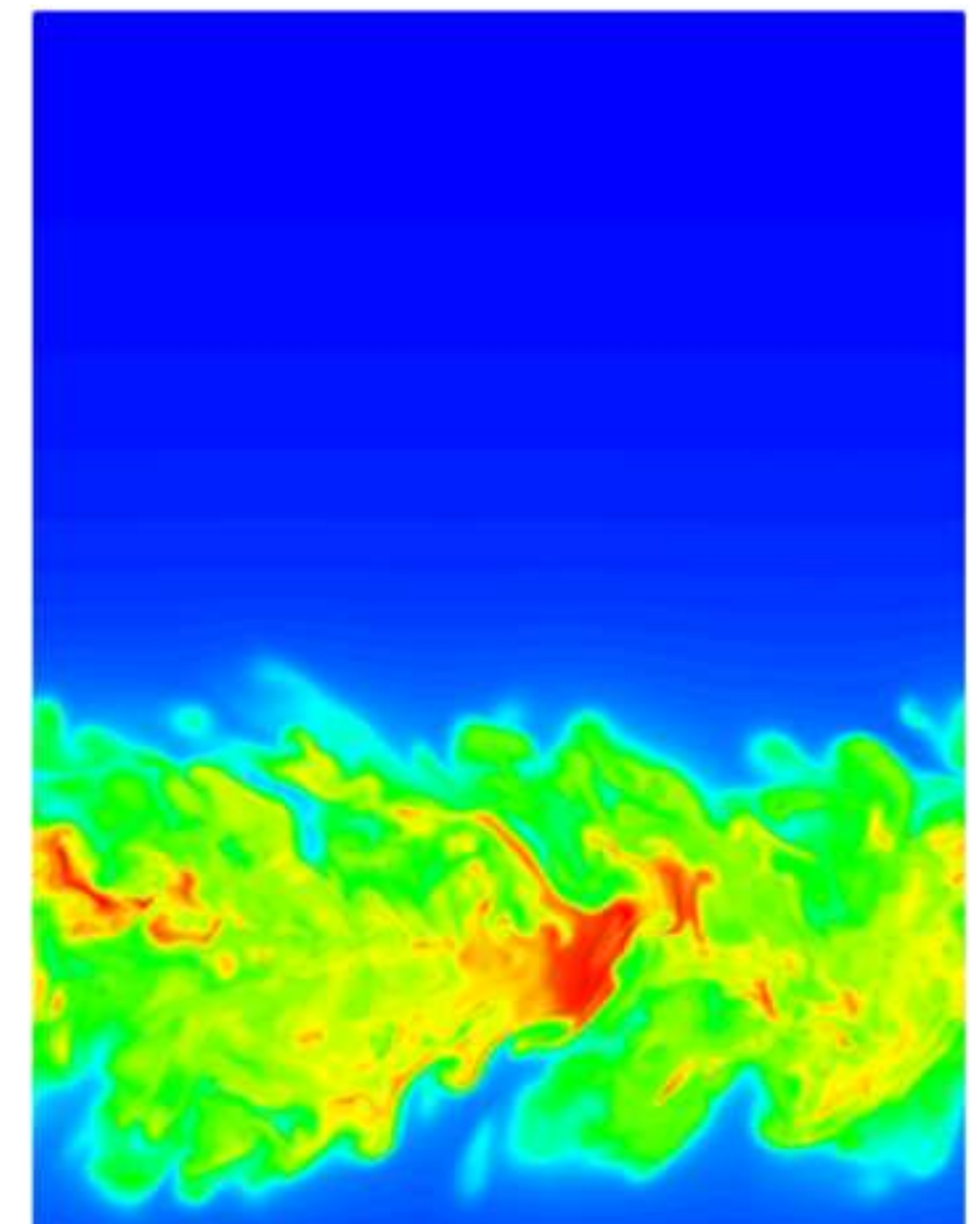


Figure - $6 b$

CO

8.6E-02

3.1E-02

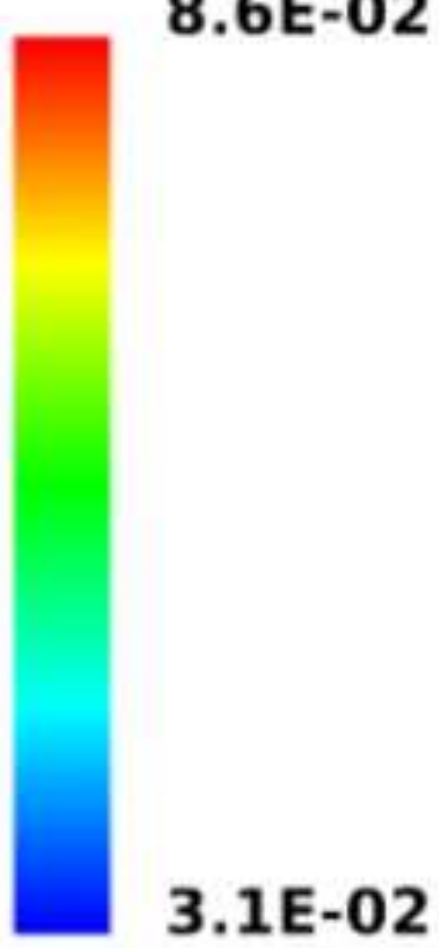


Figure - $6 c$

H2

\subsection{E-02}

6.8E-04
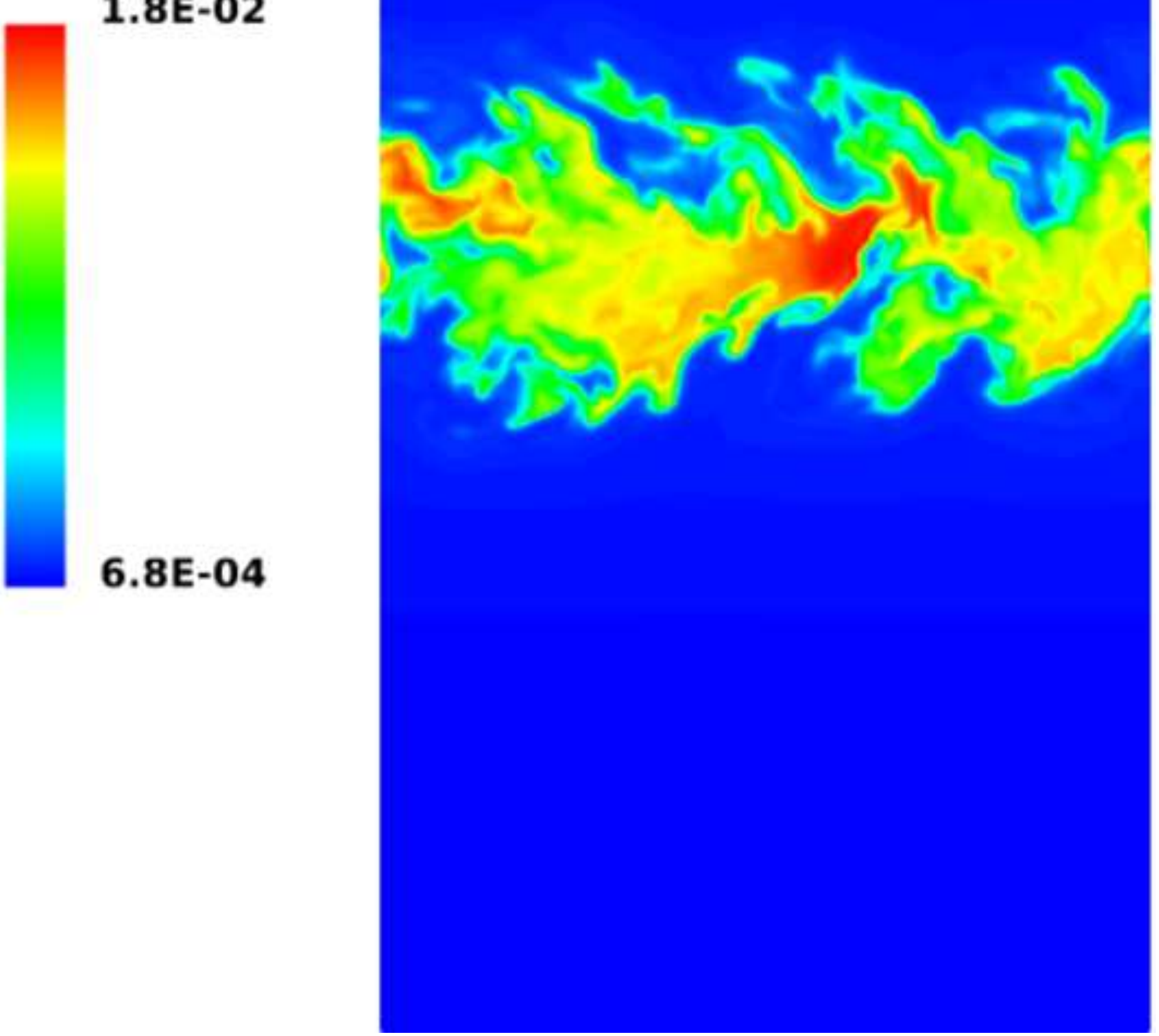


\section{H2}

2.1E-03

6.0E-04 
Figure - 7a

\section{2}

\subsection{E-01}

2.3E-02

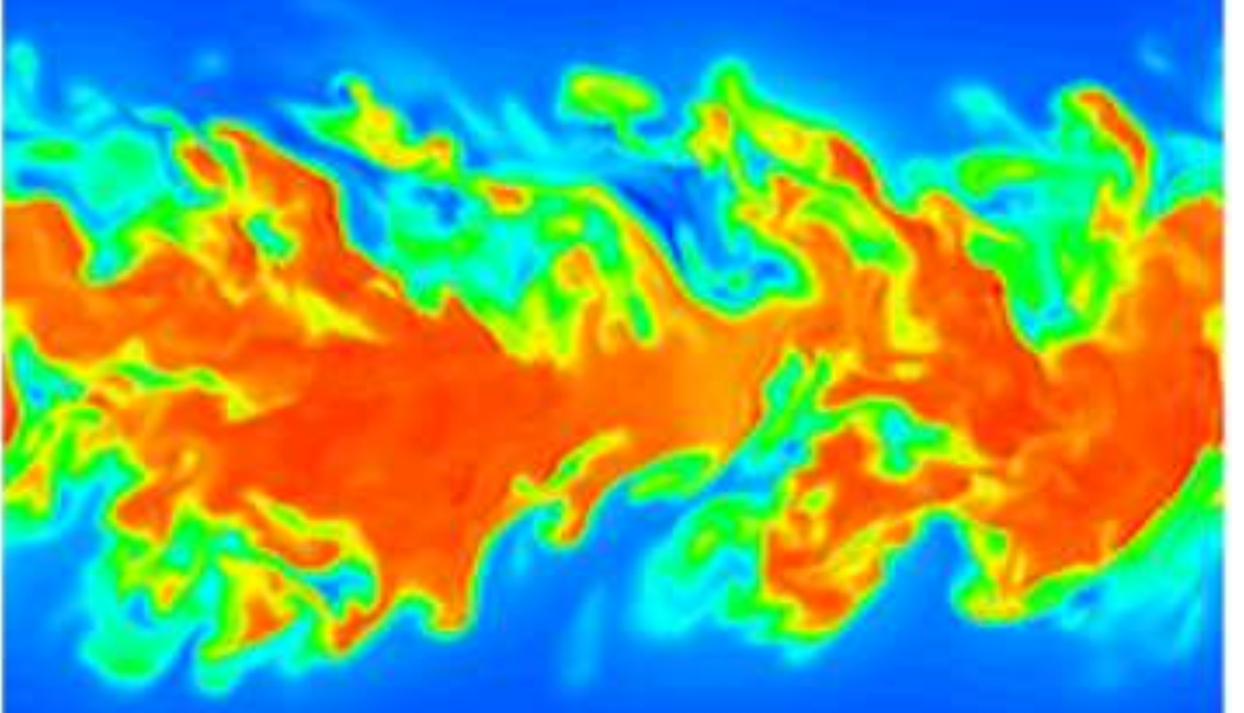




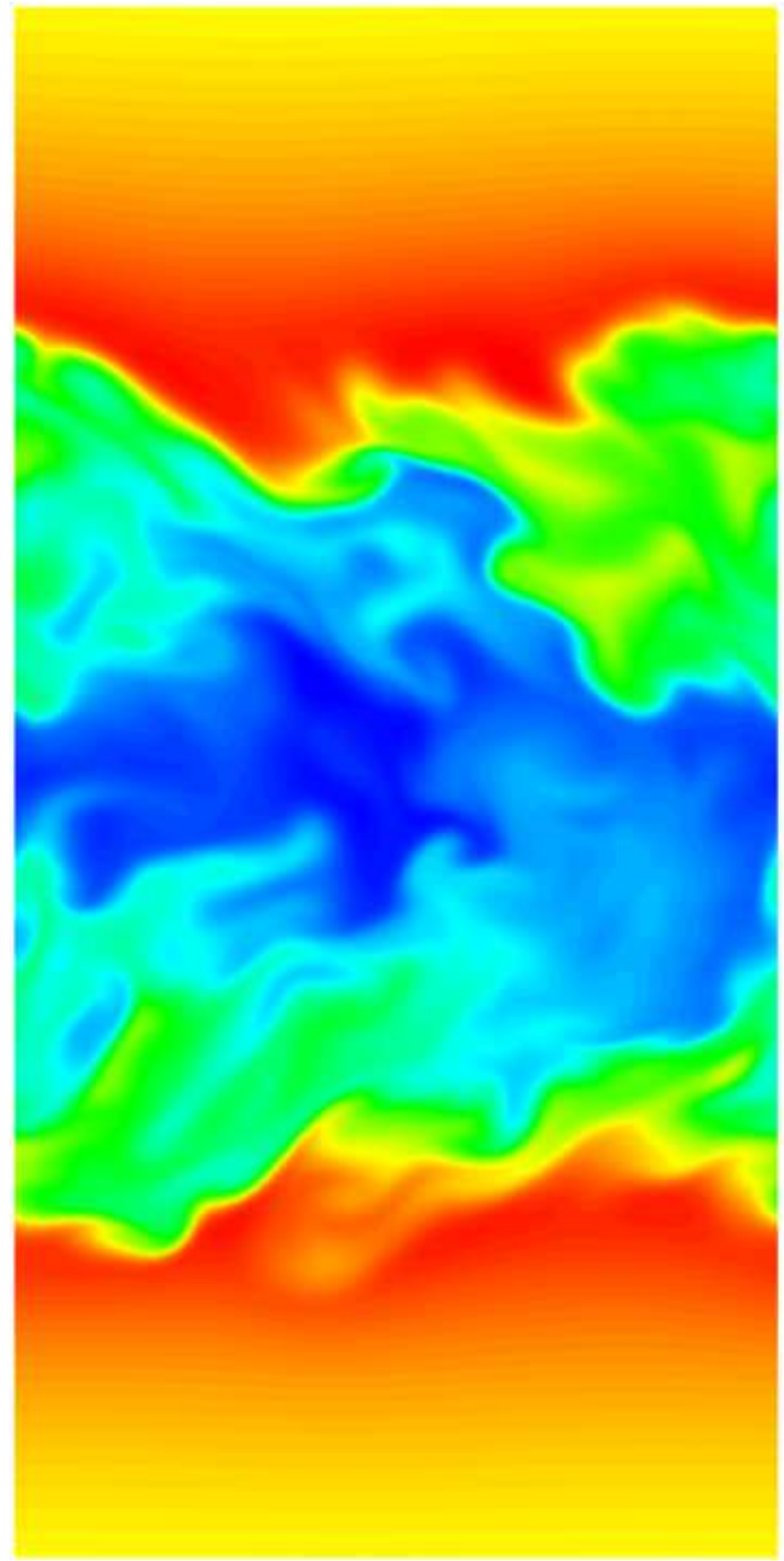

\subsection{E-02}

8.5E-03 
Figure - 7c

\section{H2O}

\subsection{E-02}

$0.0 E+00$

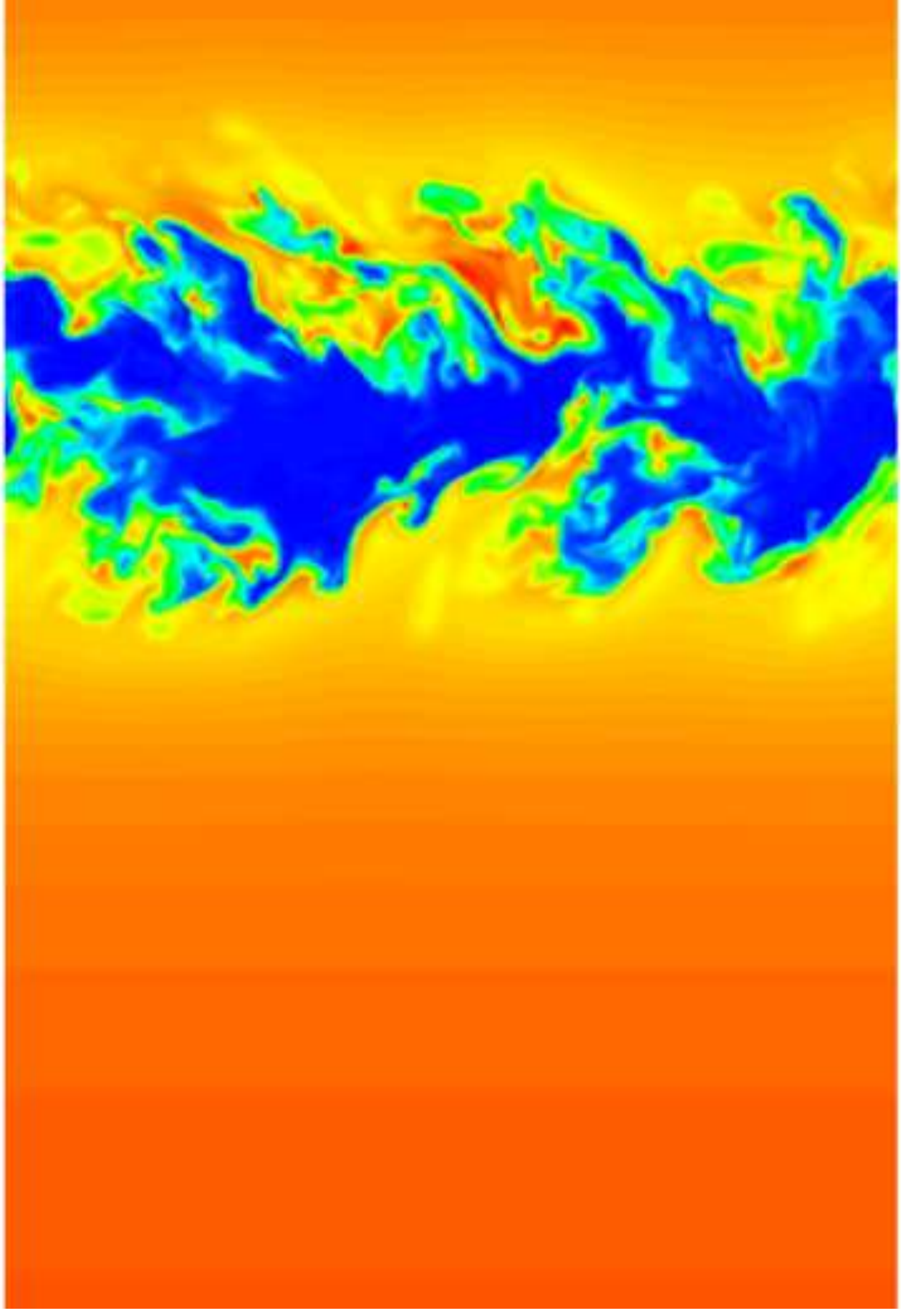




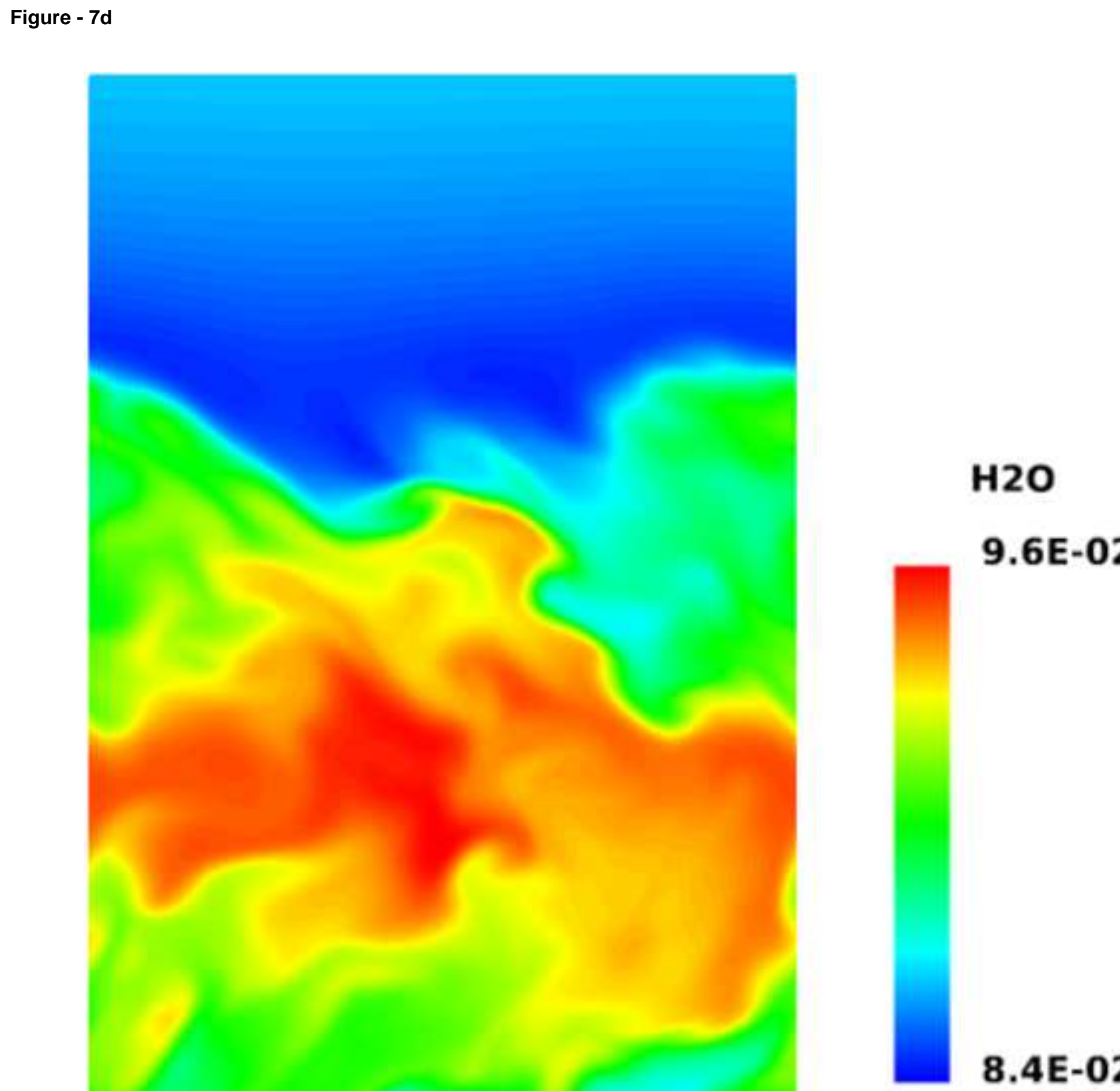

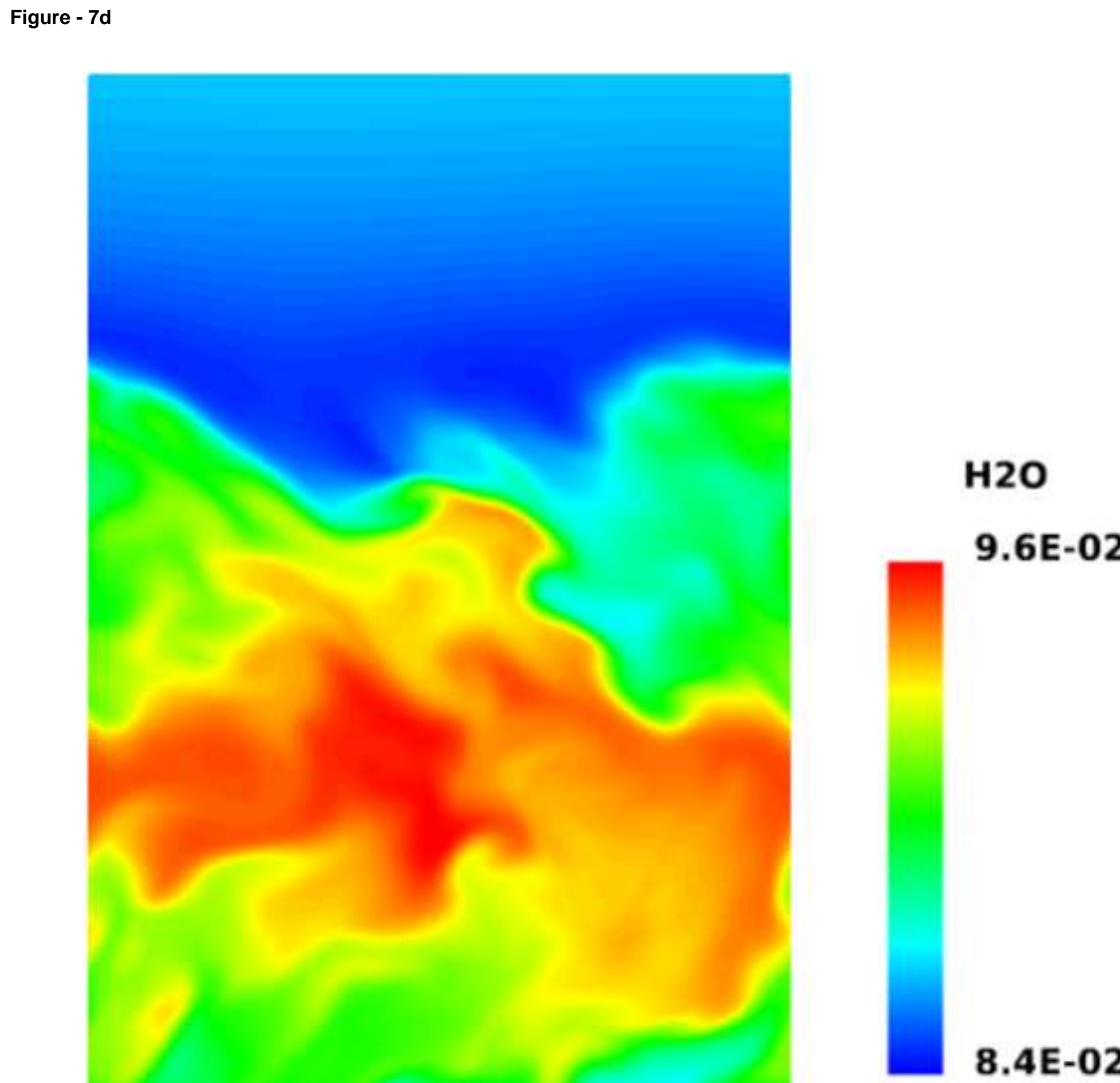

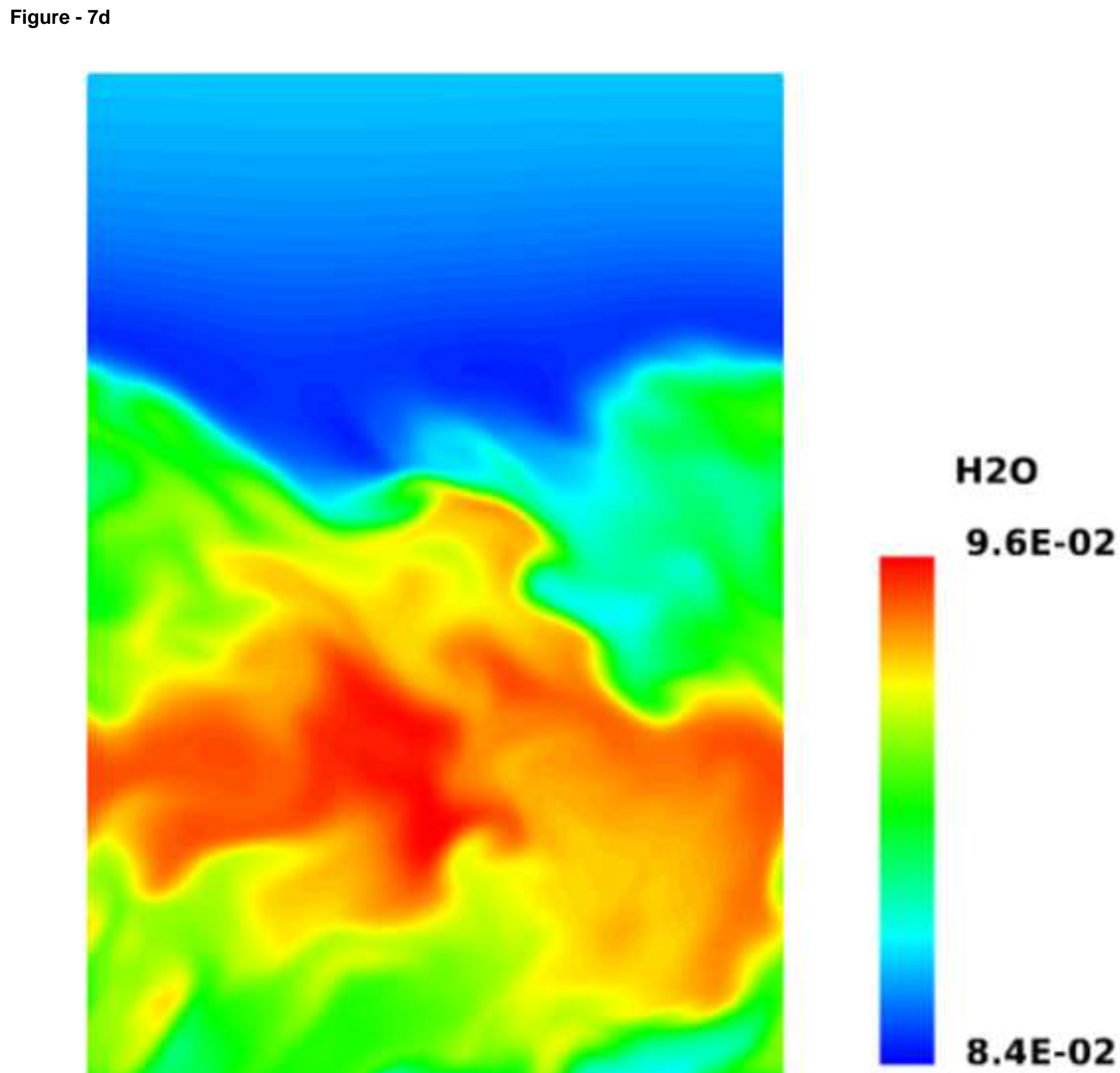

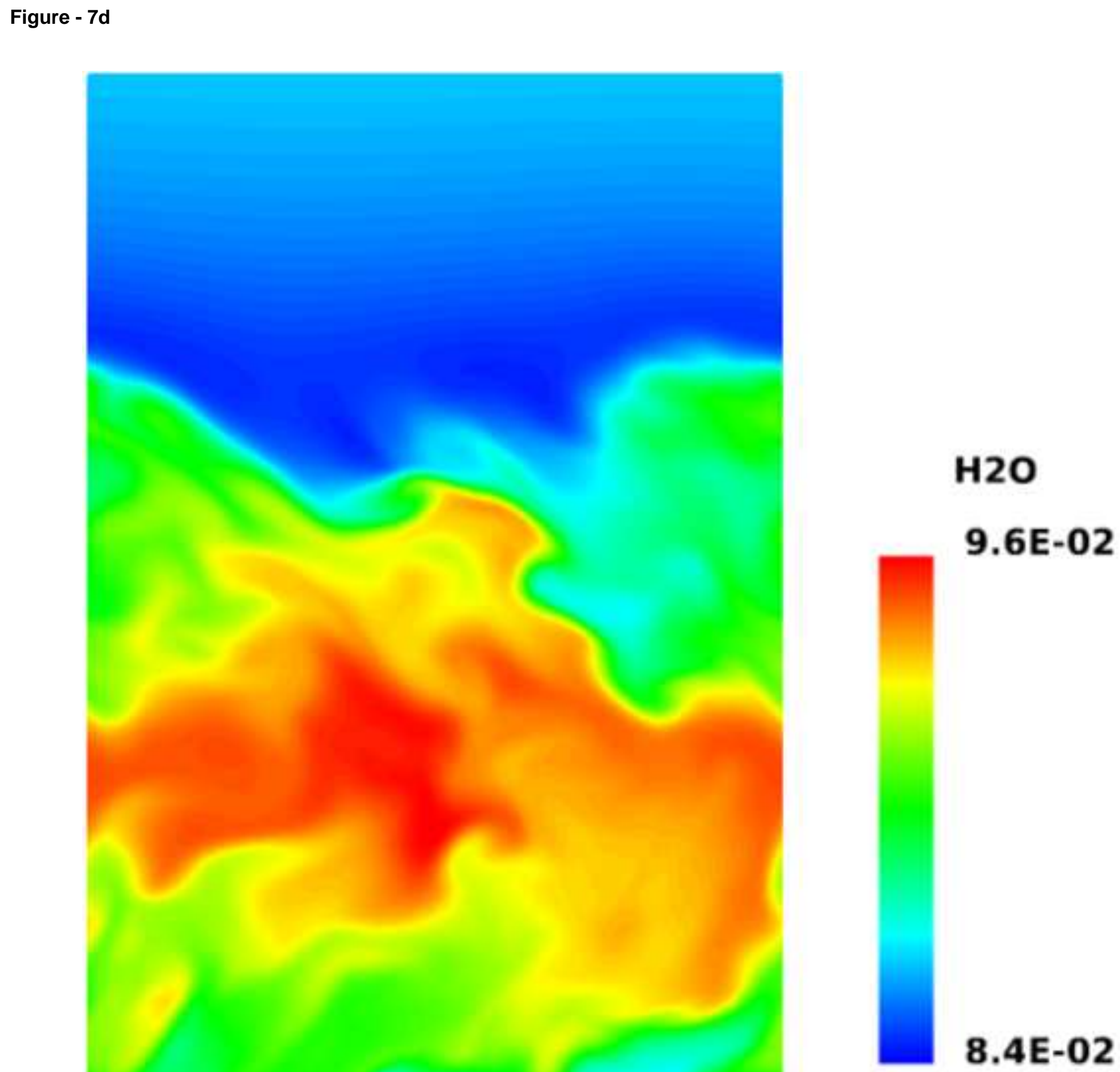
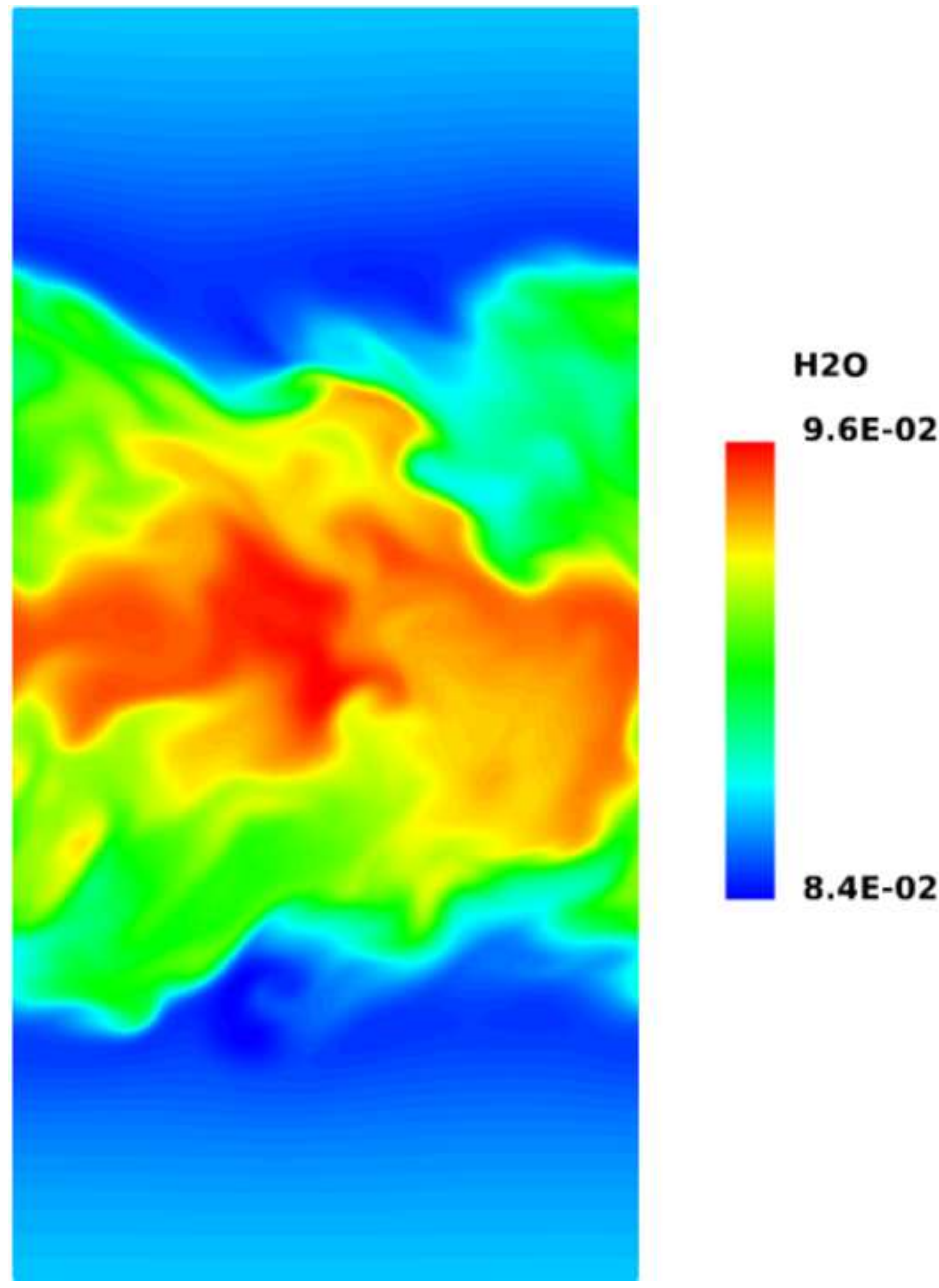

.

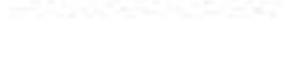




\section{OH}

9.1E-03

\section{$0.0 E+00$}

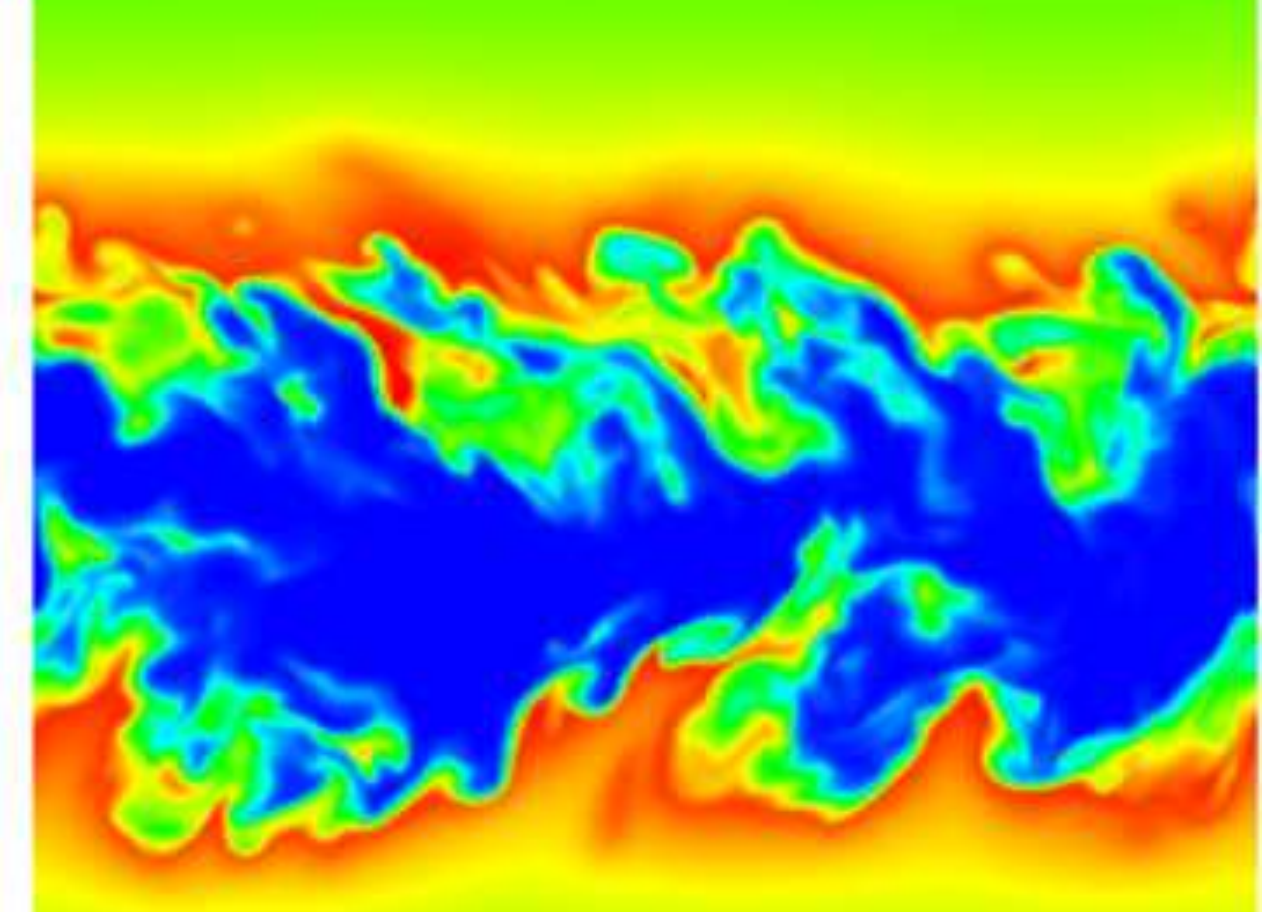

Figure - $8 a$

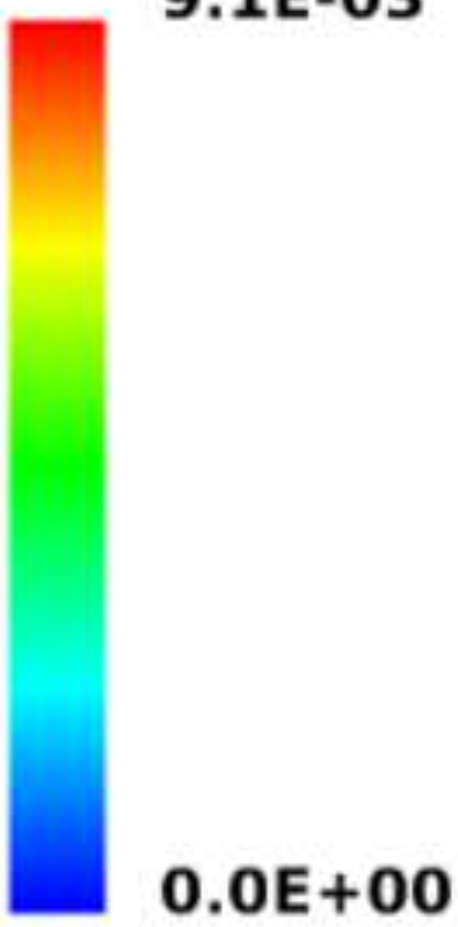



Figure - 8b

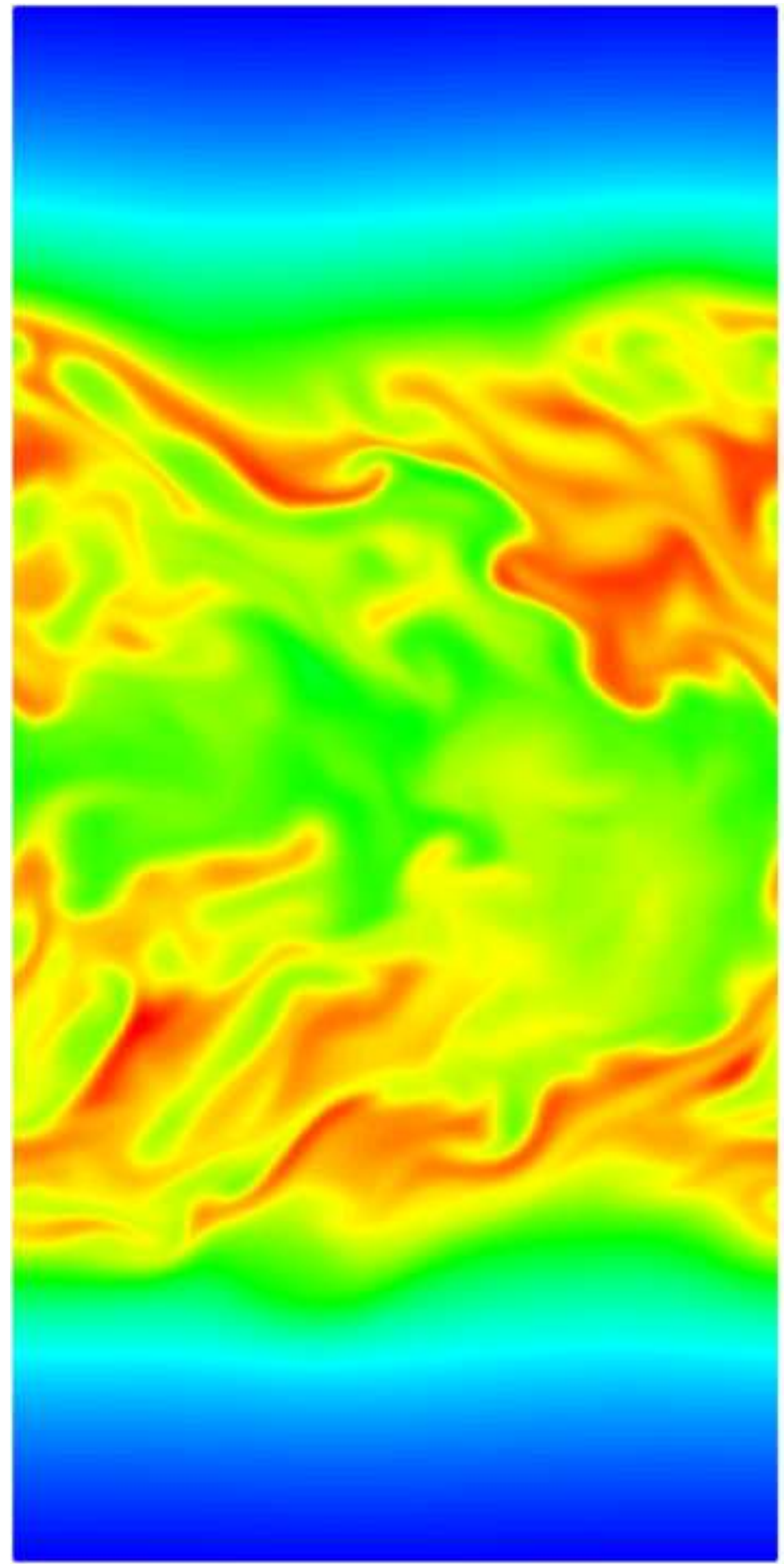

$\mathrm{OH}$

5.1E-03

3.7E-03

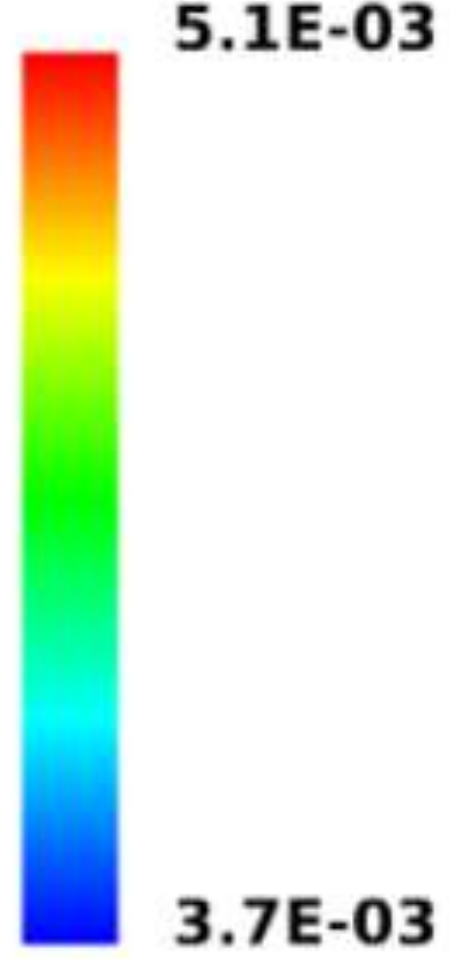


Figure - 8c

H

\subsection{E-03}

$0.0 E+00$
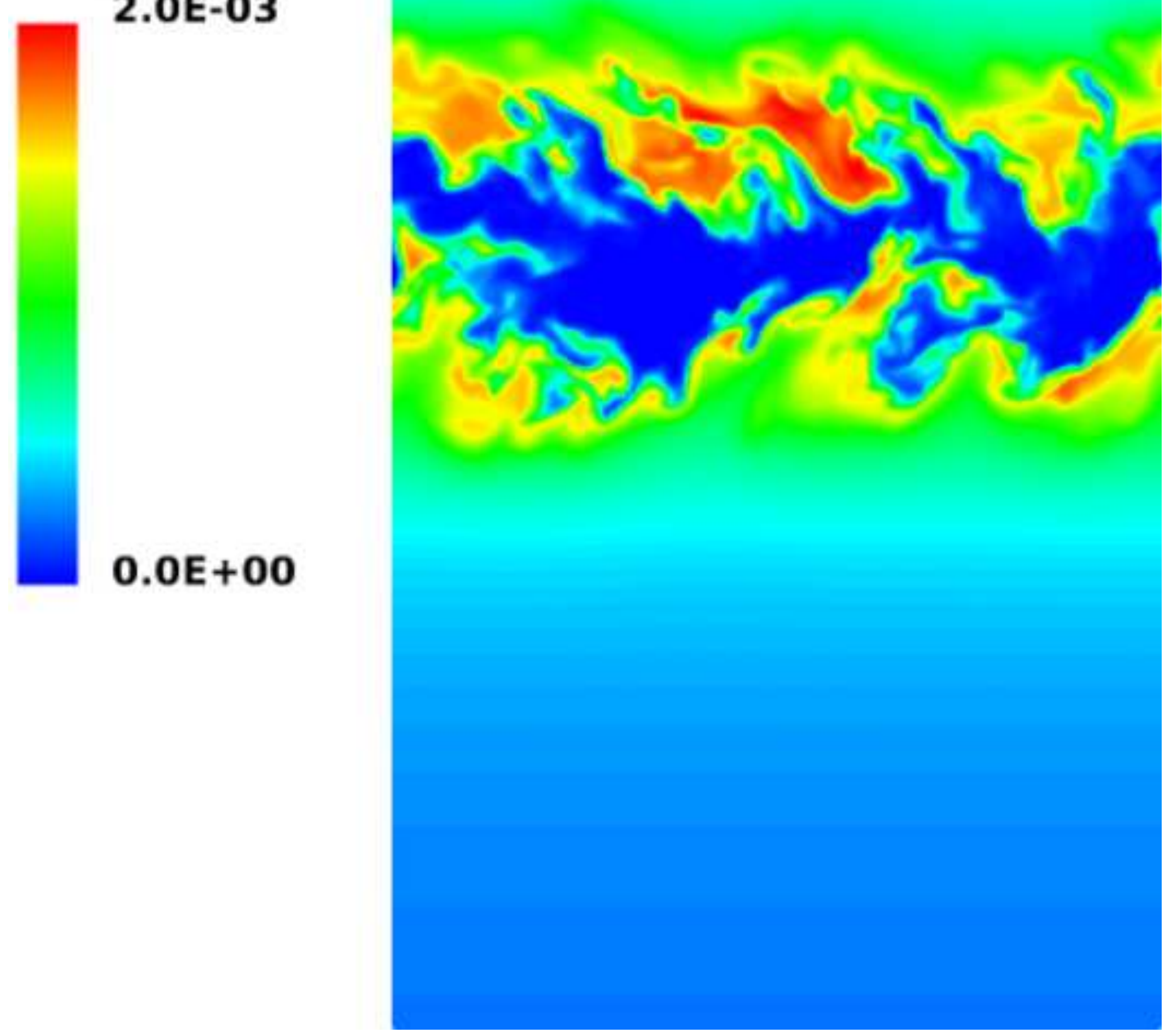
Figure - 8d

1.8E-04
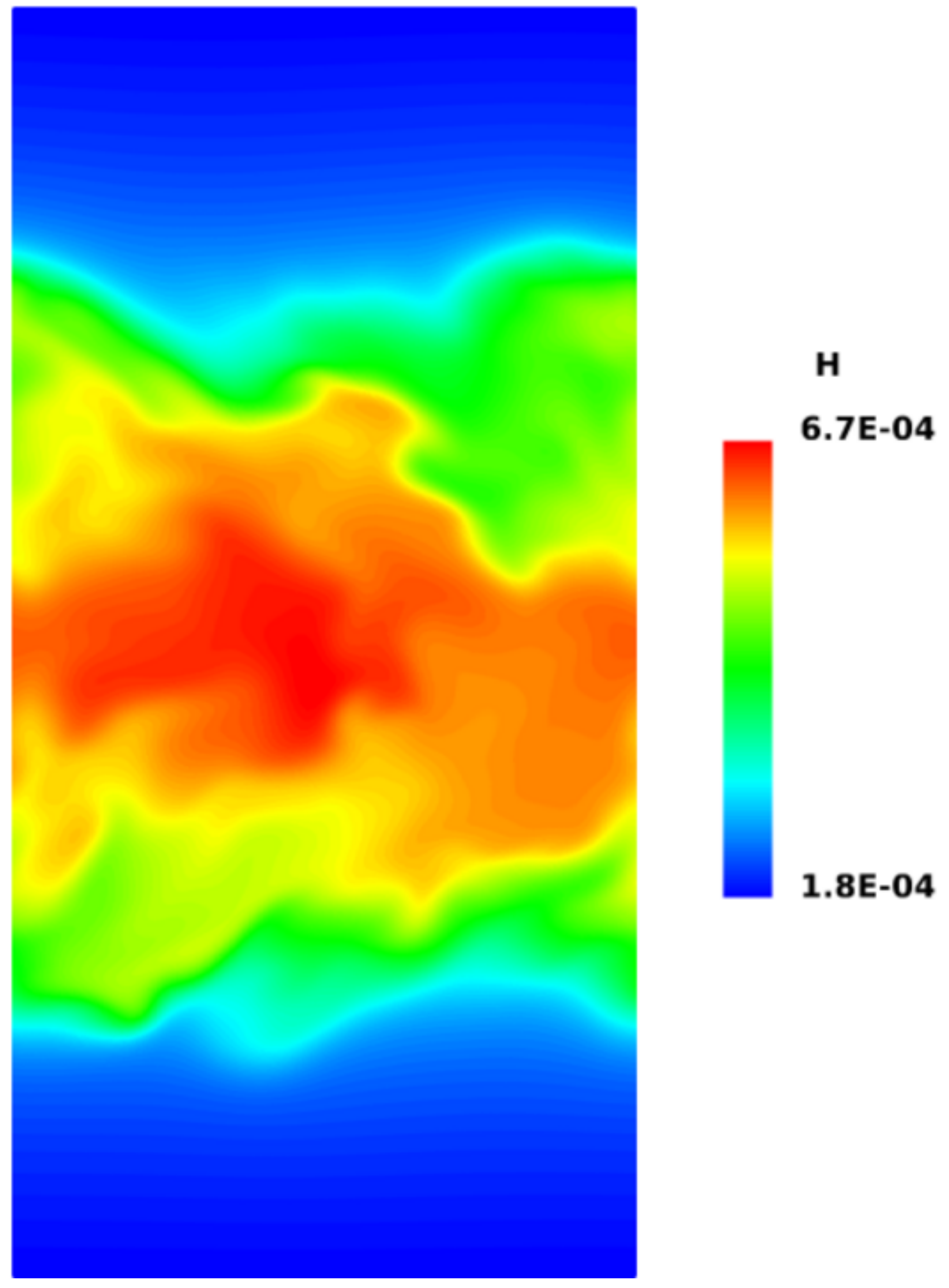

\section{(1)}

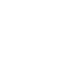

\section{H \\ 6.7E-04}


Figure - 9a

o

\subsection{E-02}

$0.0 E+00$

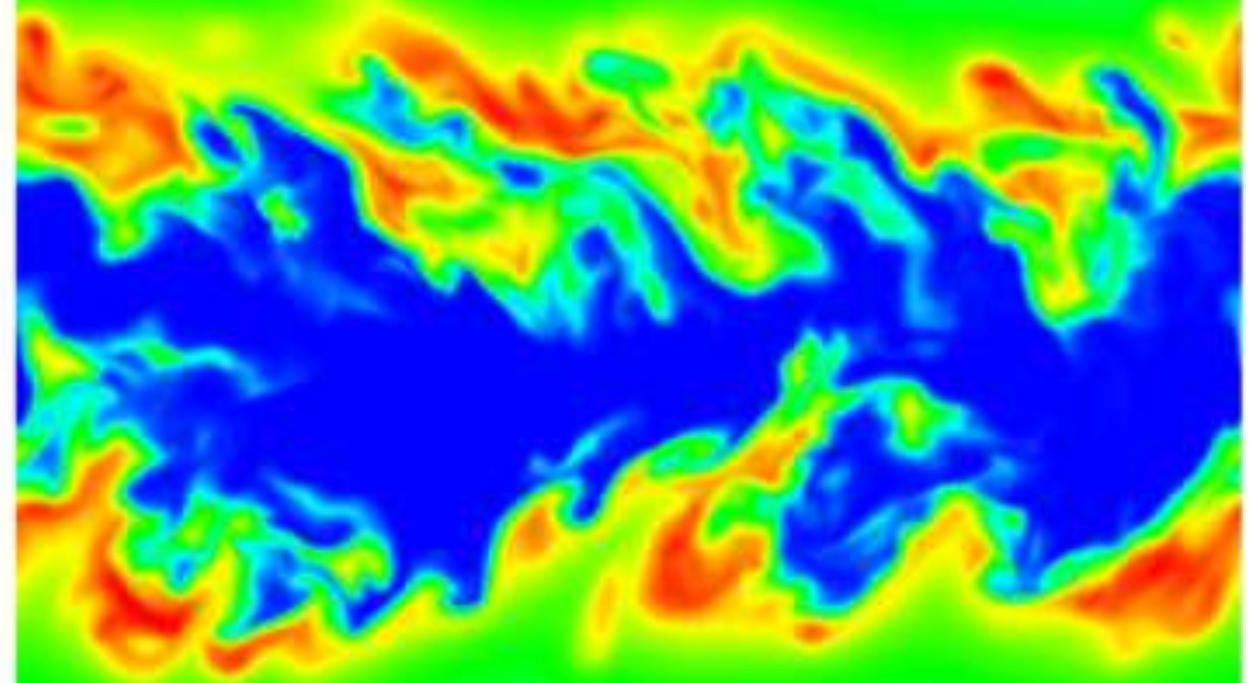


Figure - 9b

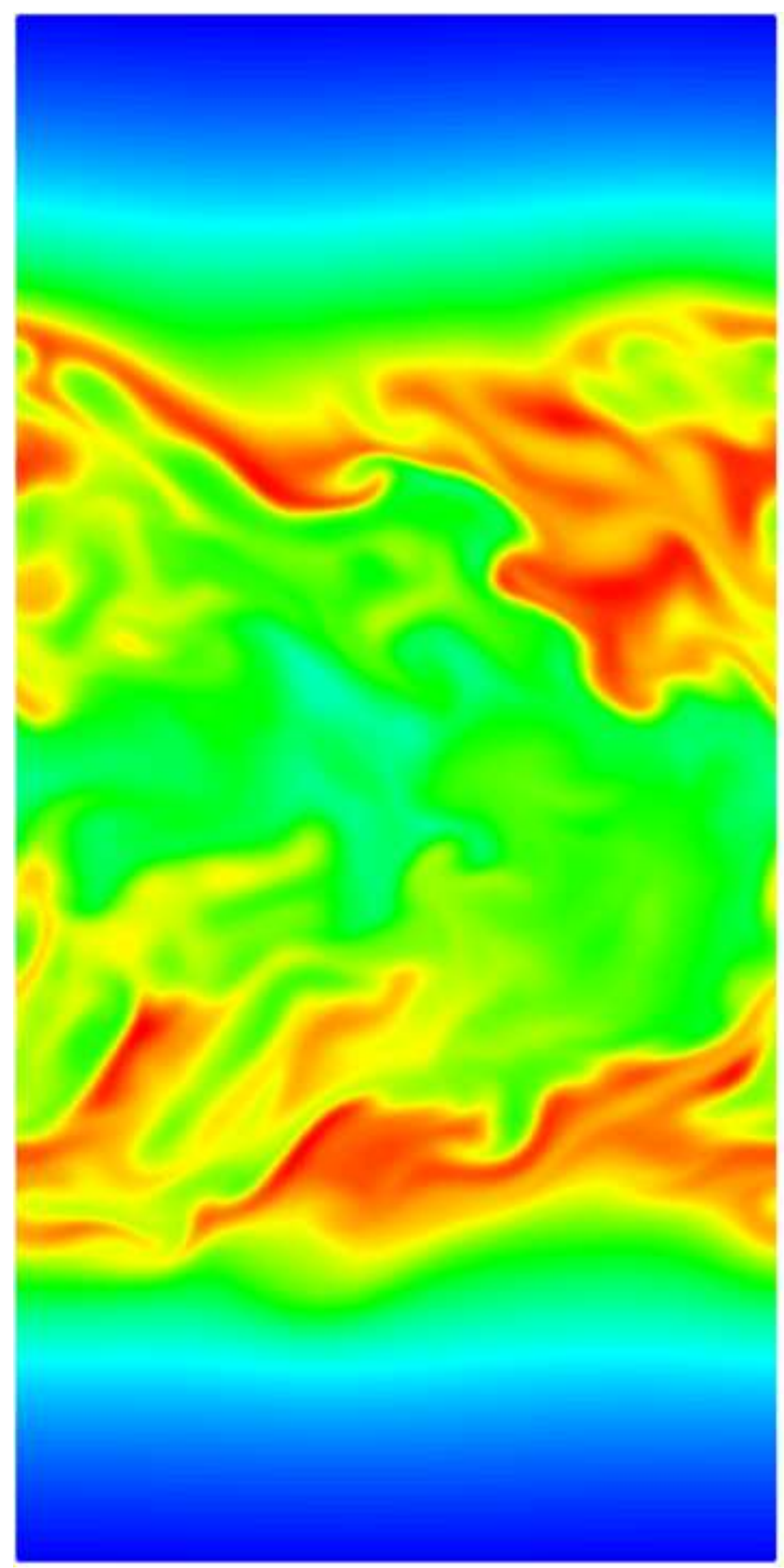

1.4E-03

\section{0 \\ 2.5E-03}

(1)

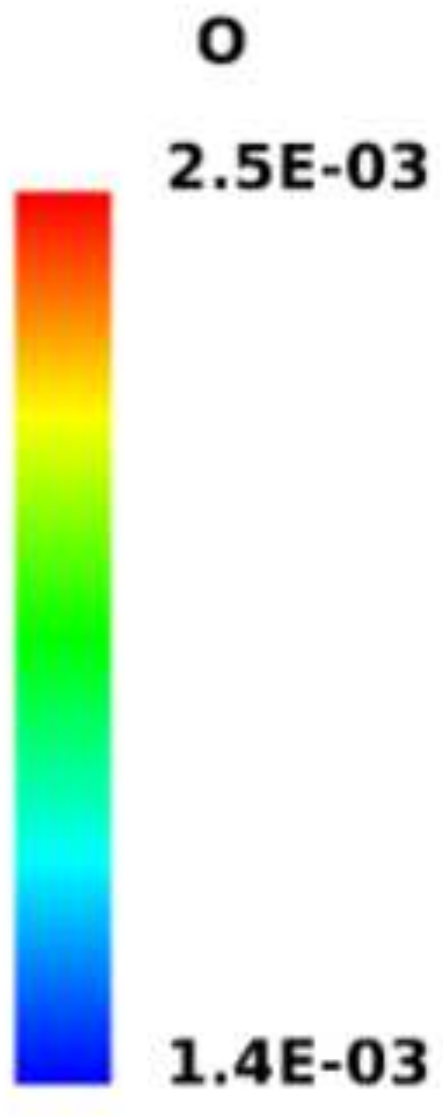

.


Figure - 9d

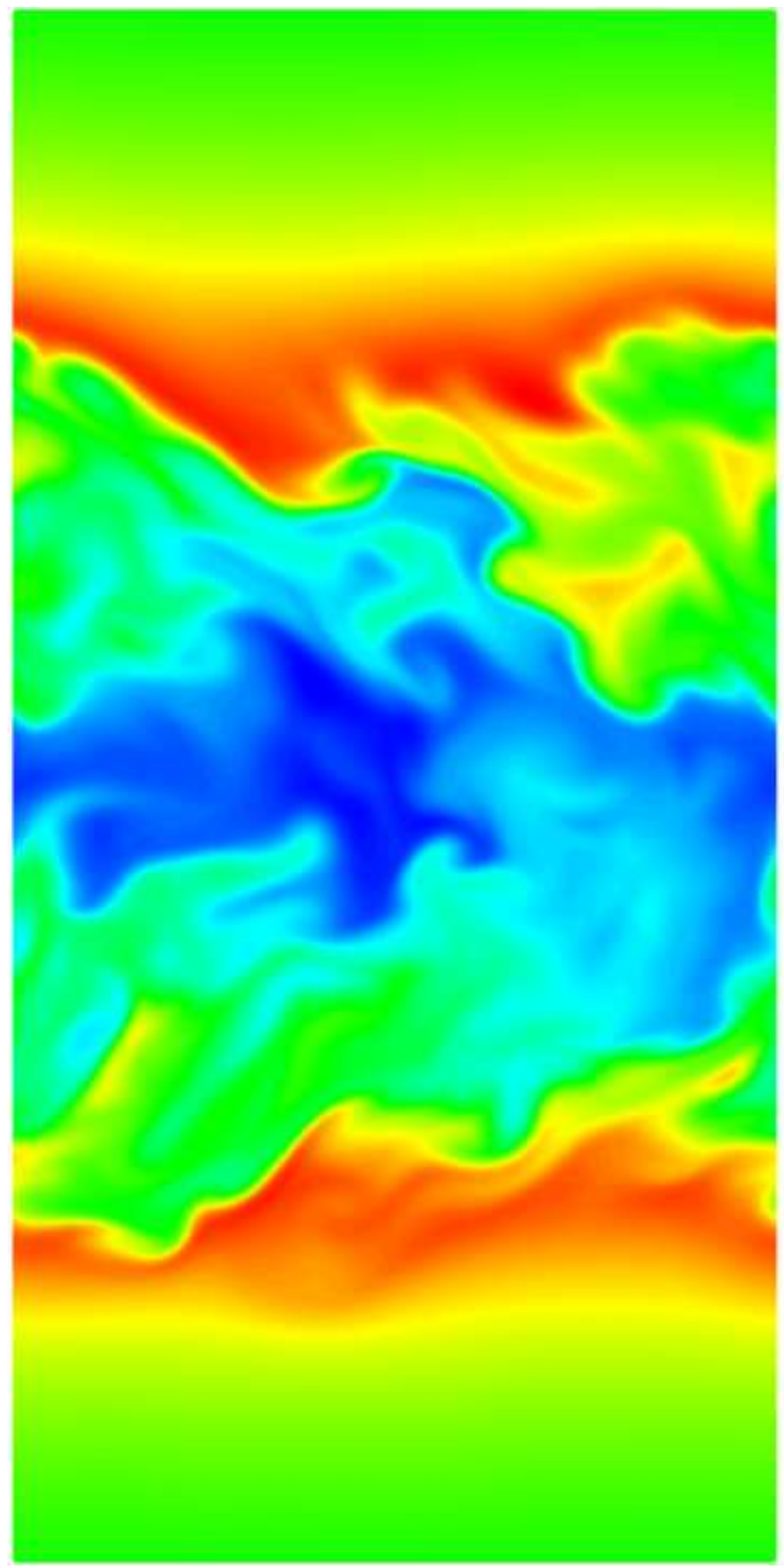

\section{$\mathrm{HO2}$}

4.6E-06

2.2E-06

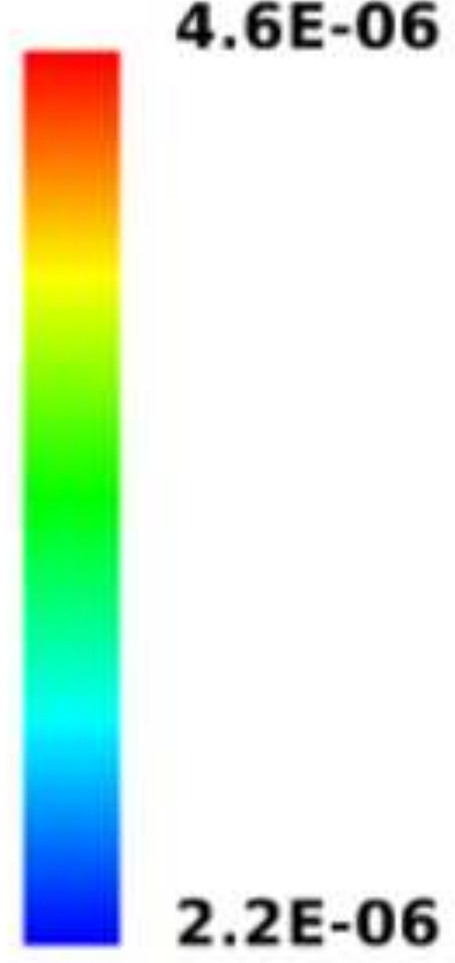



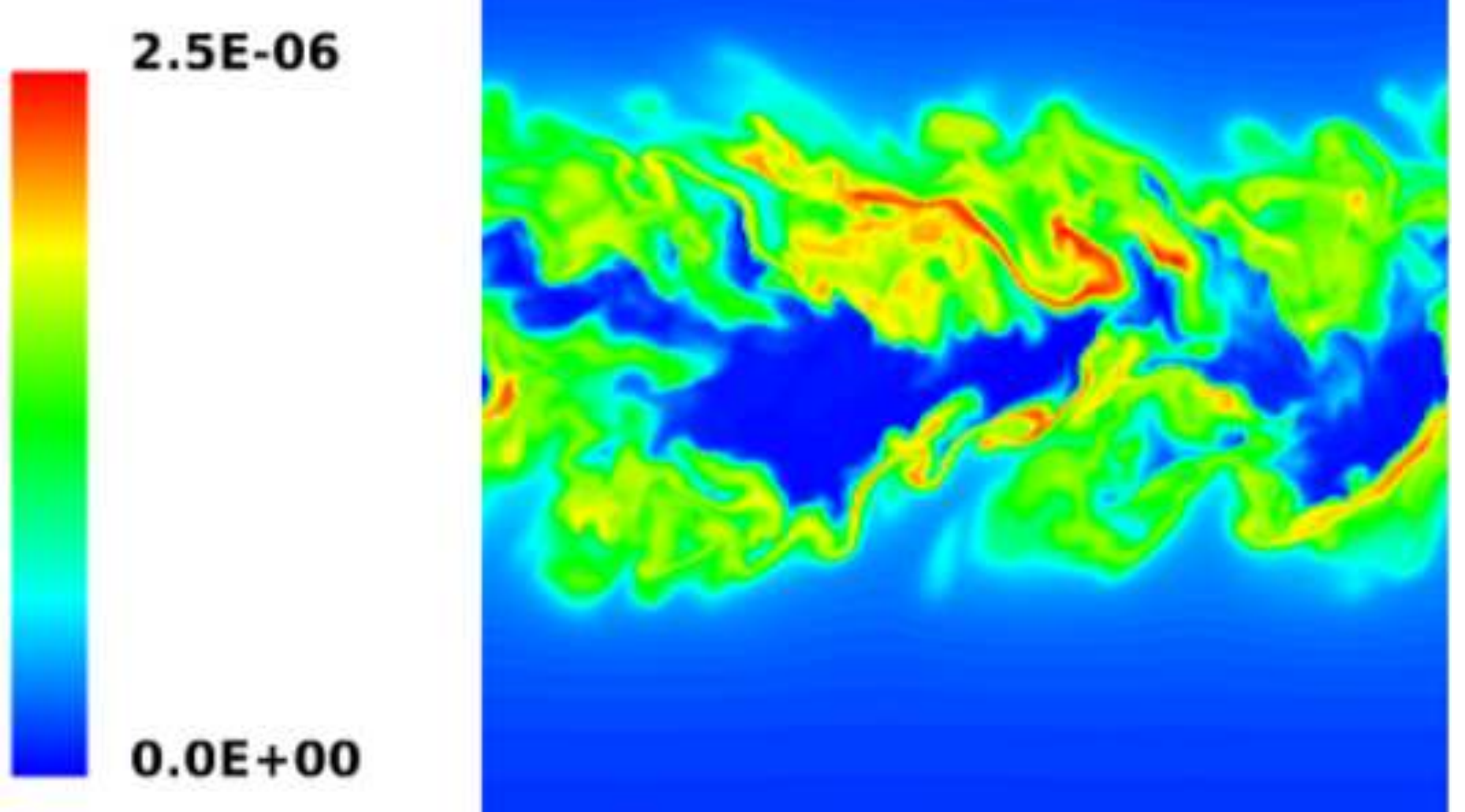

\section{HCO}

\subsection{E-06}

\section{$0.0 E+00$}

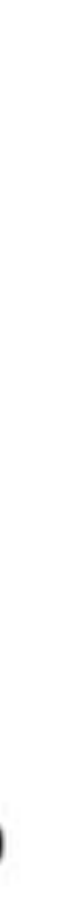

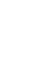
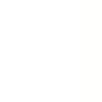


\section{HCO}

\subsection{E-07}

5.1E-08
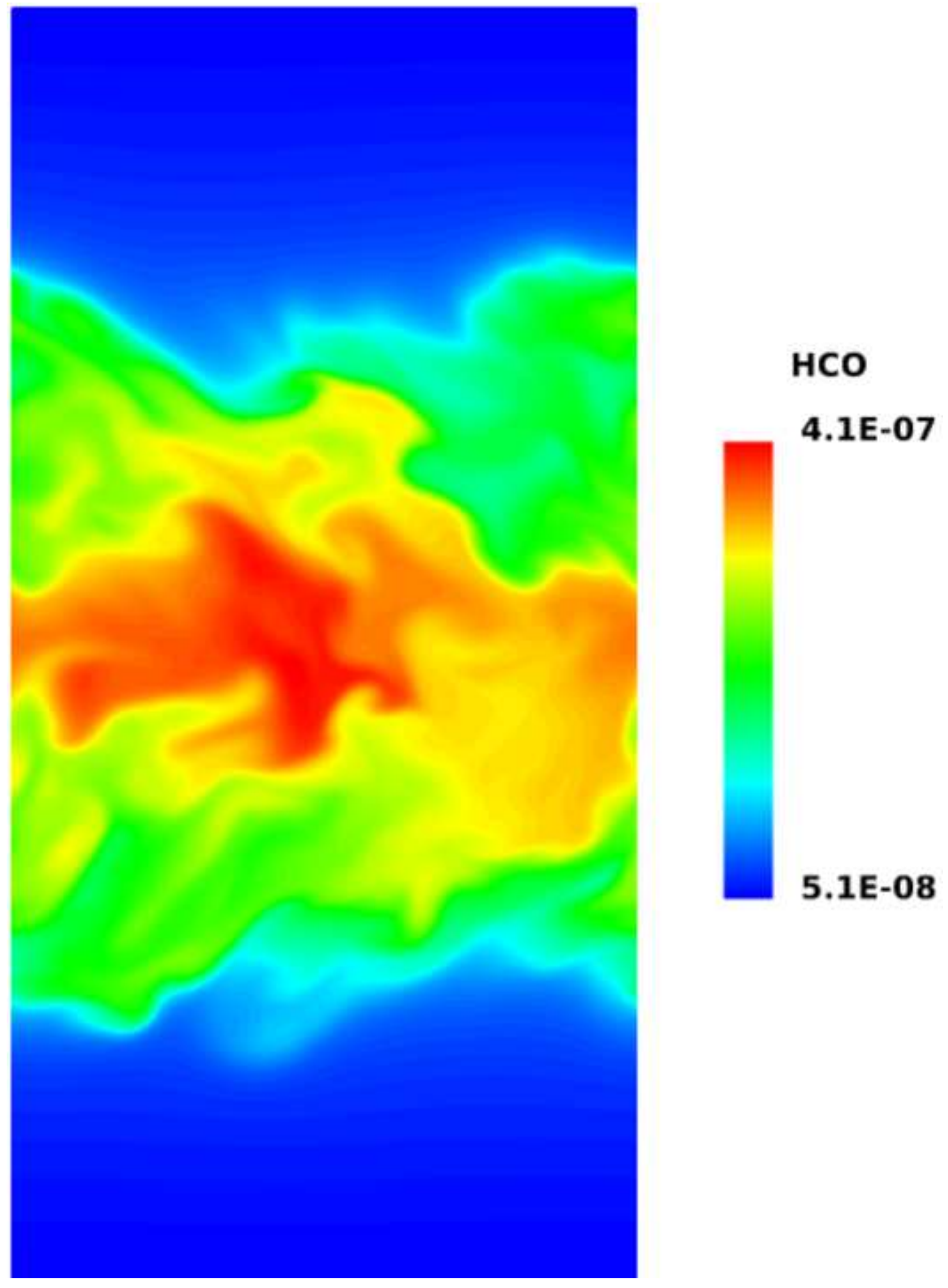


\section{$0.0 E+00$}
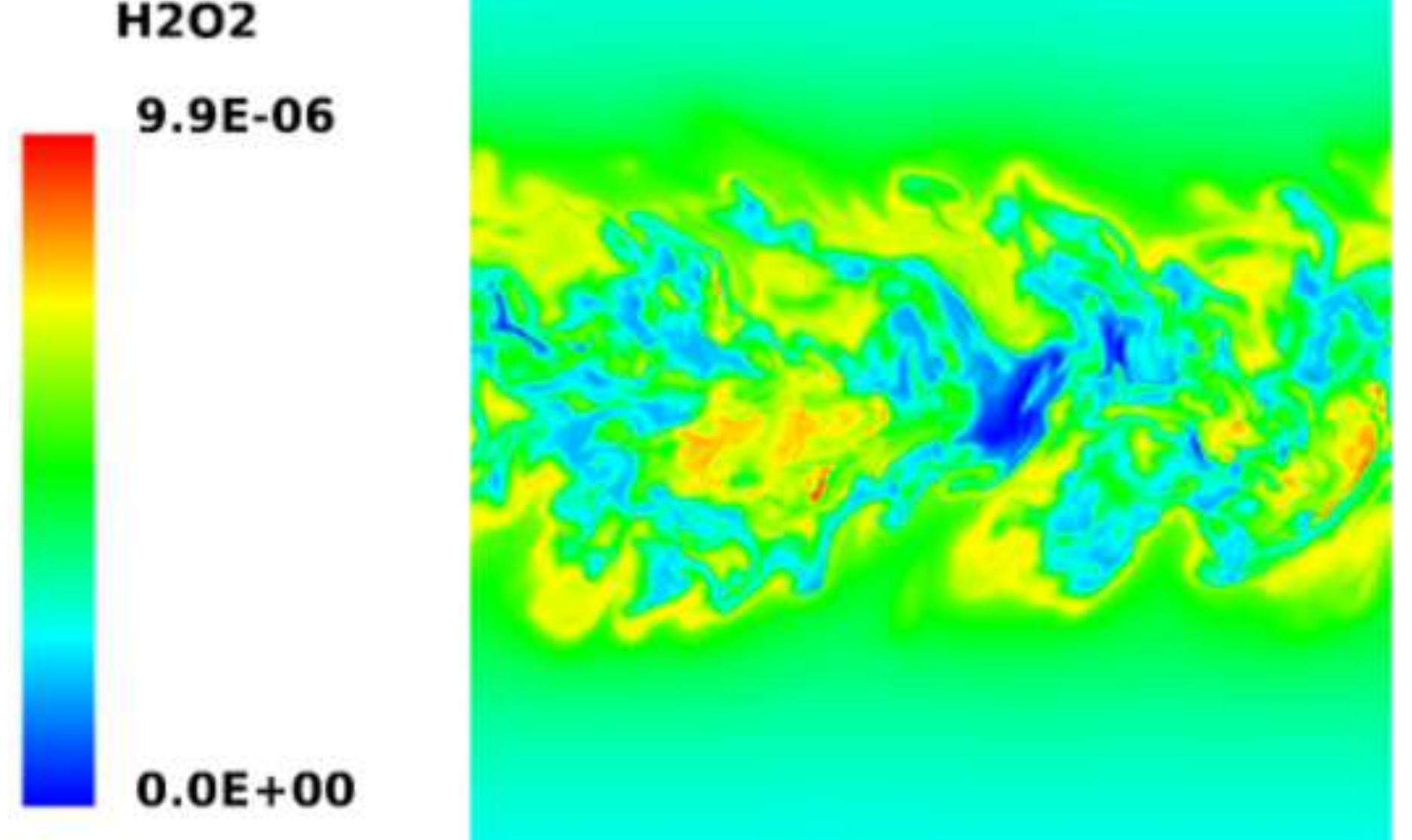

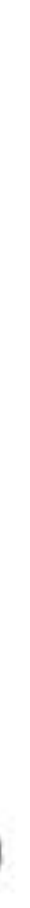

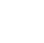

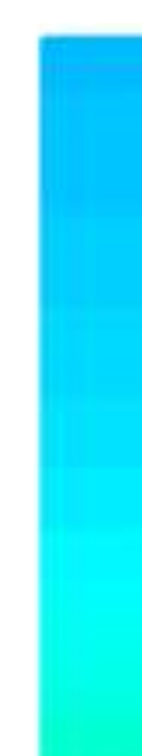

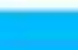

10

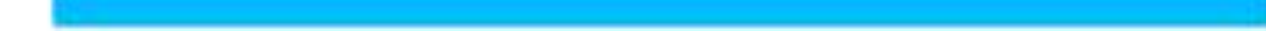




\section{H2O2}

\subsection{E-06}

\subsection{E-06}




\section{$\mathrm{CO} 2$}

\subsection{E-01}

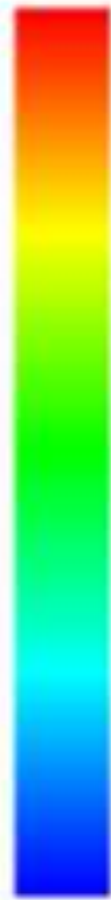

\section{$0.0 E+00$}

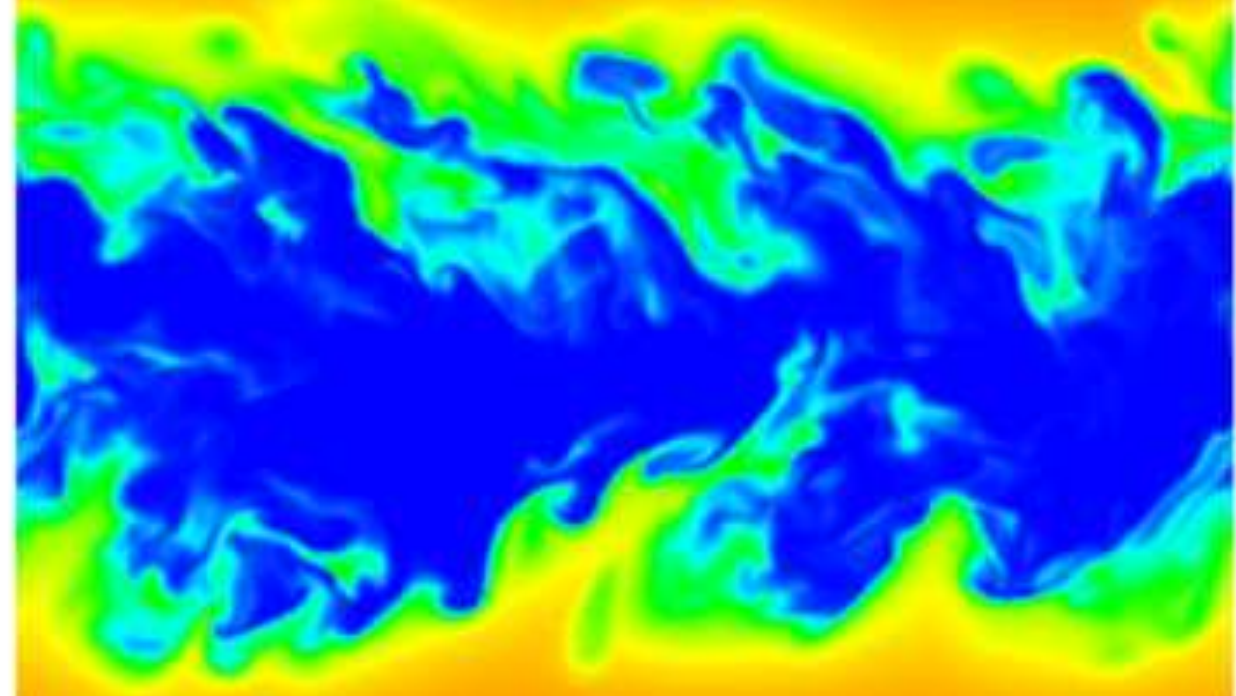


$\mathrm{CO} 2$

1.9E-01

1.7E-01

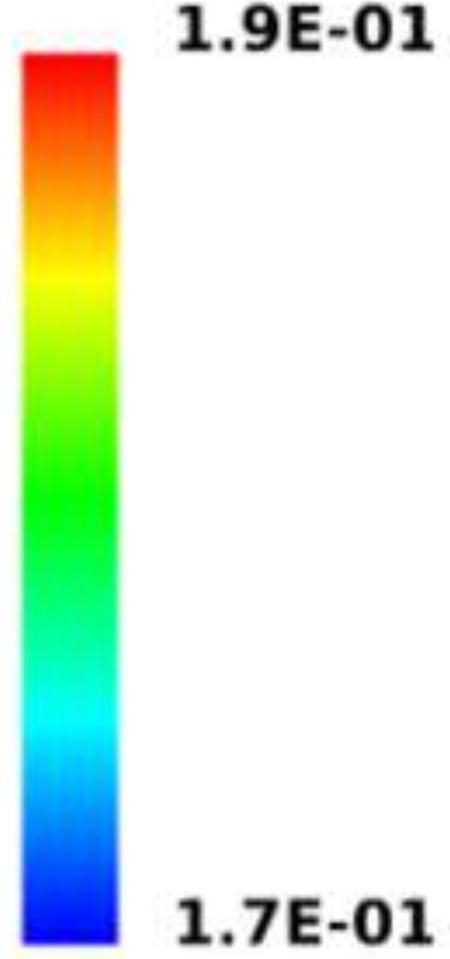


$-2.0 E+05$

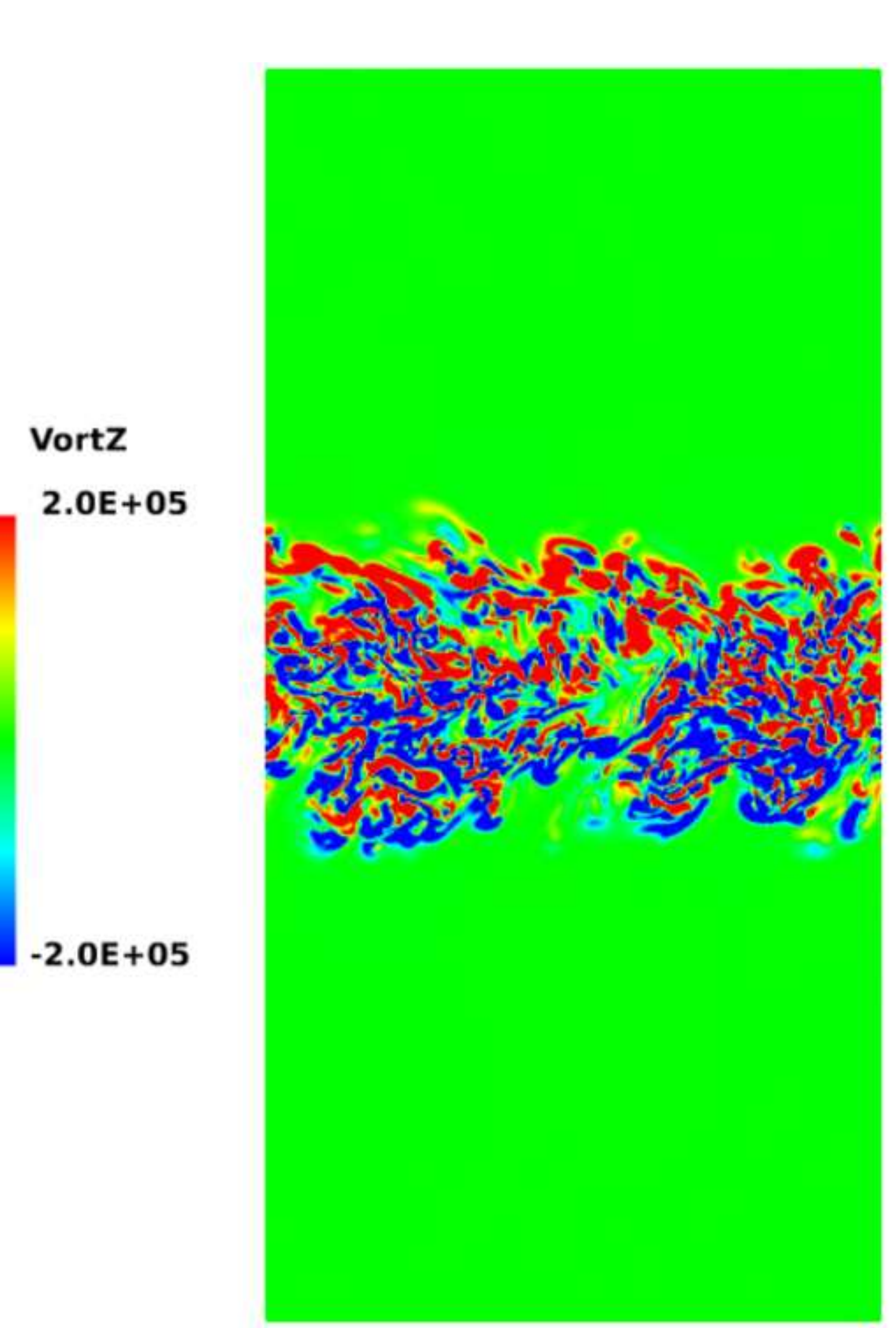

(

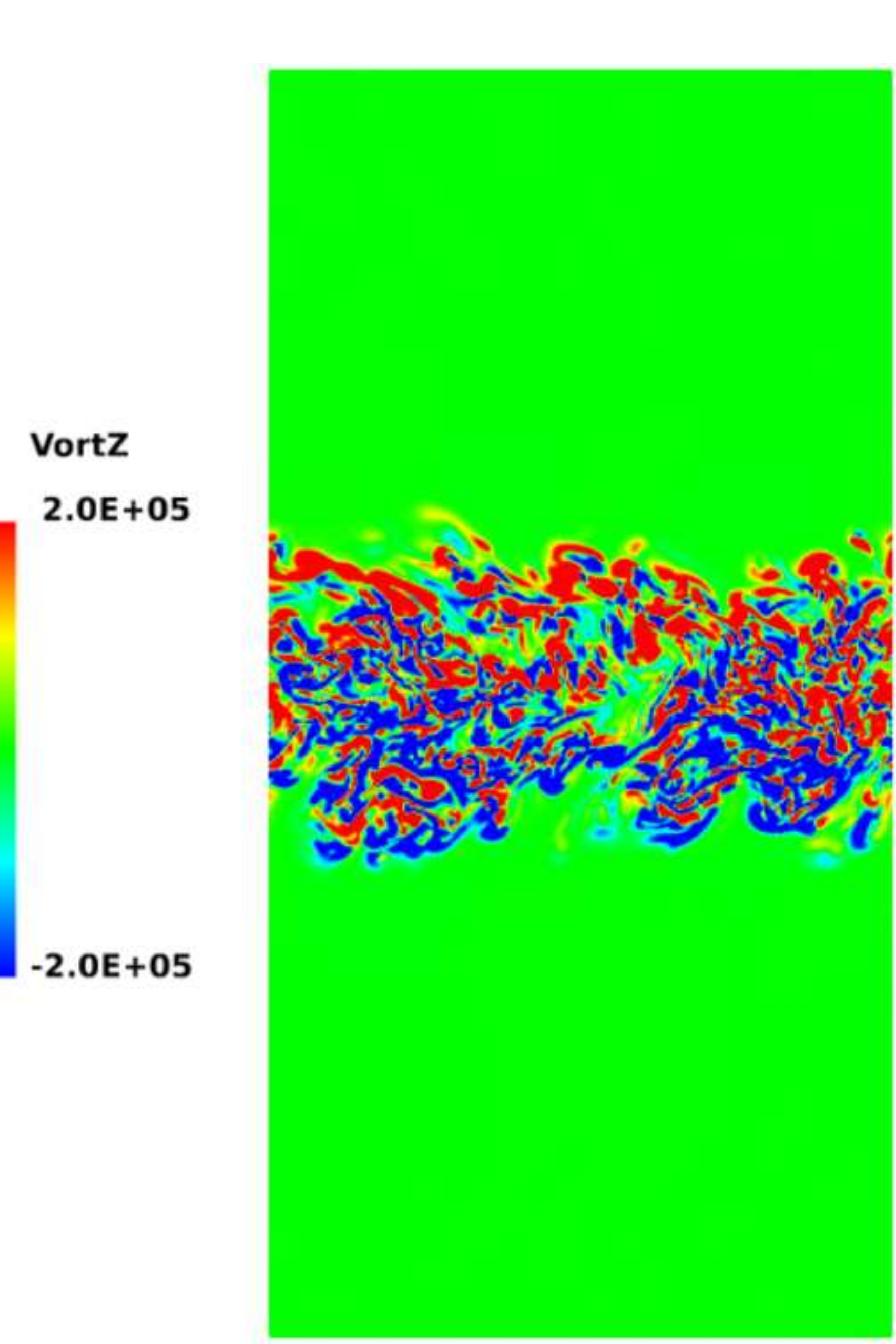

(

. 
Figure - 11d

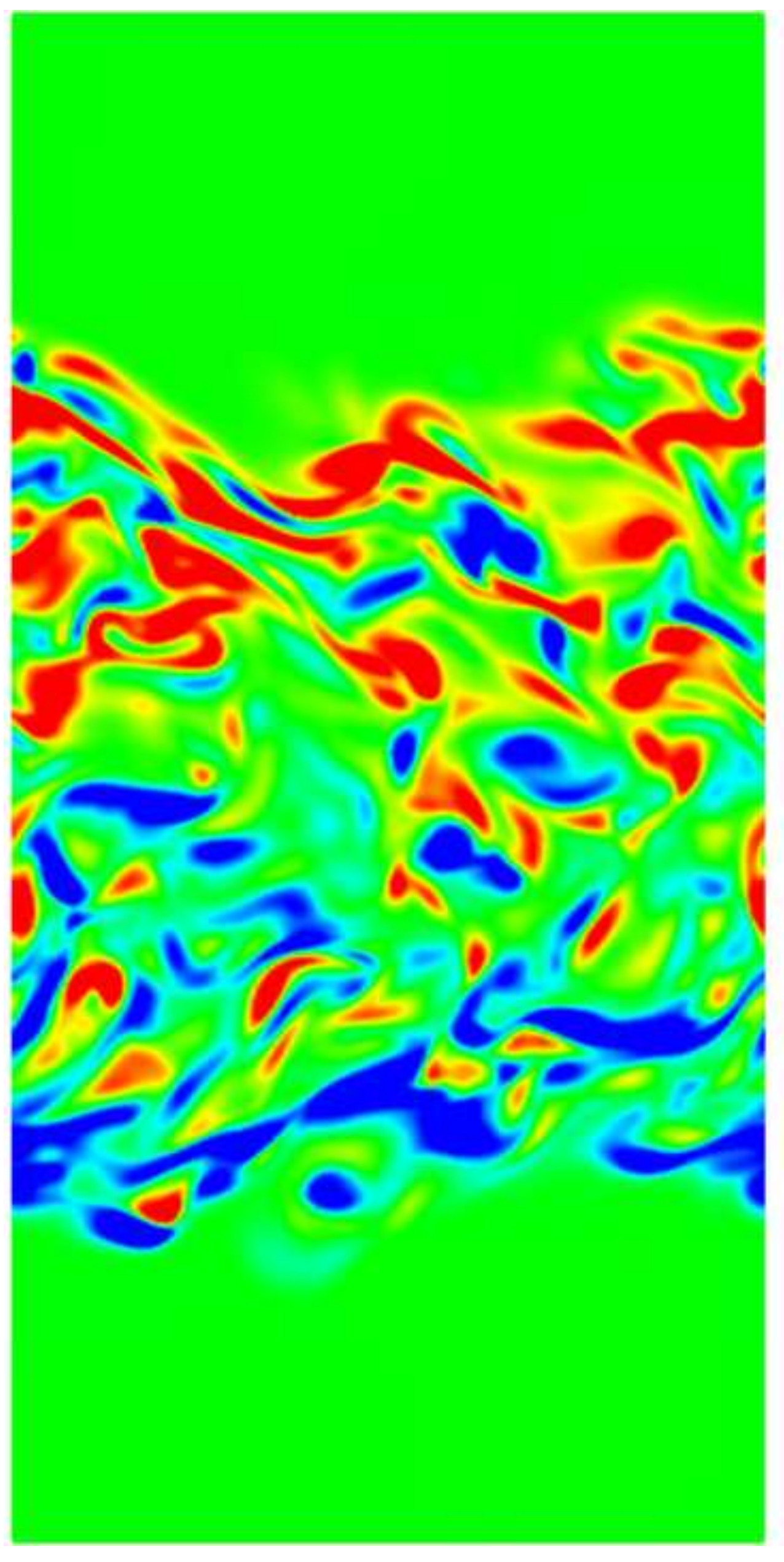

\section{VortZ}

1.0E + 05

$-1.0 E+05$

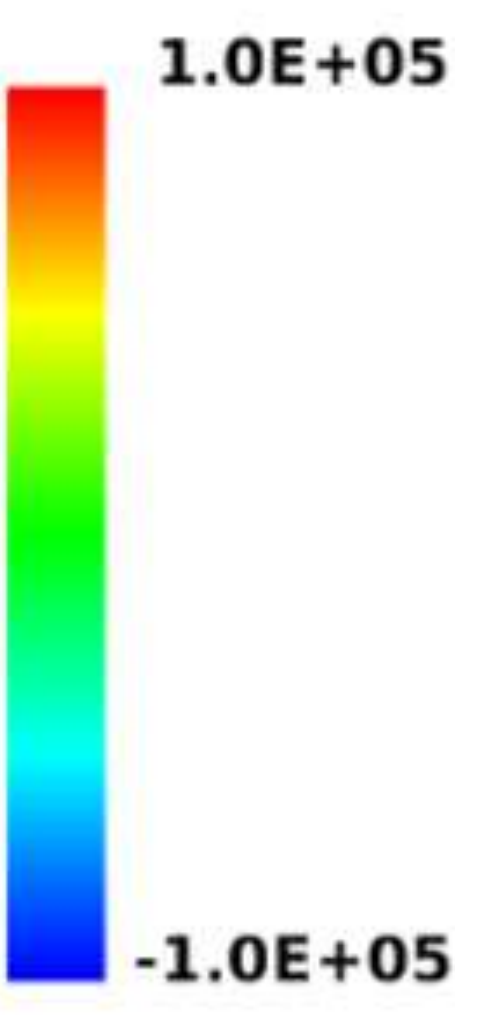




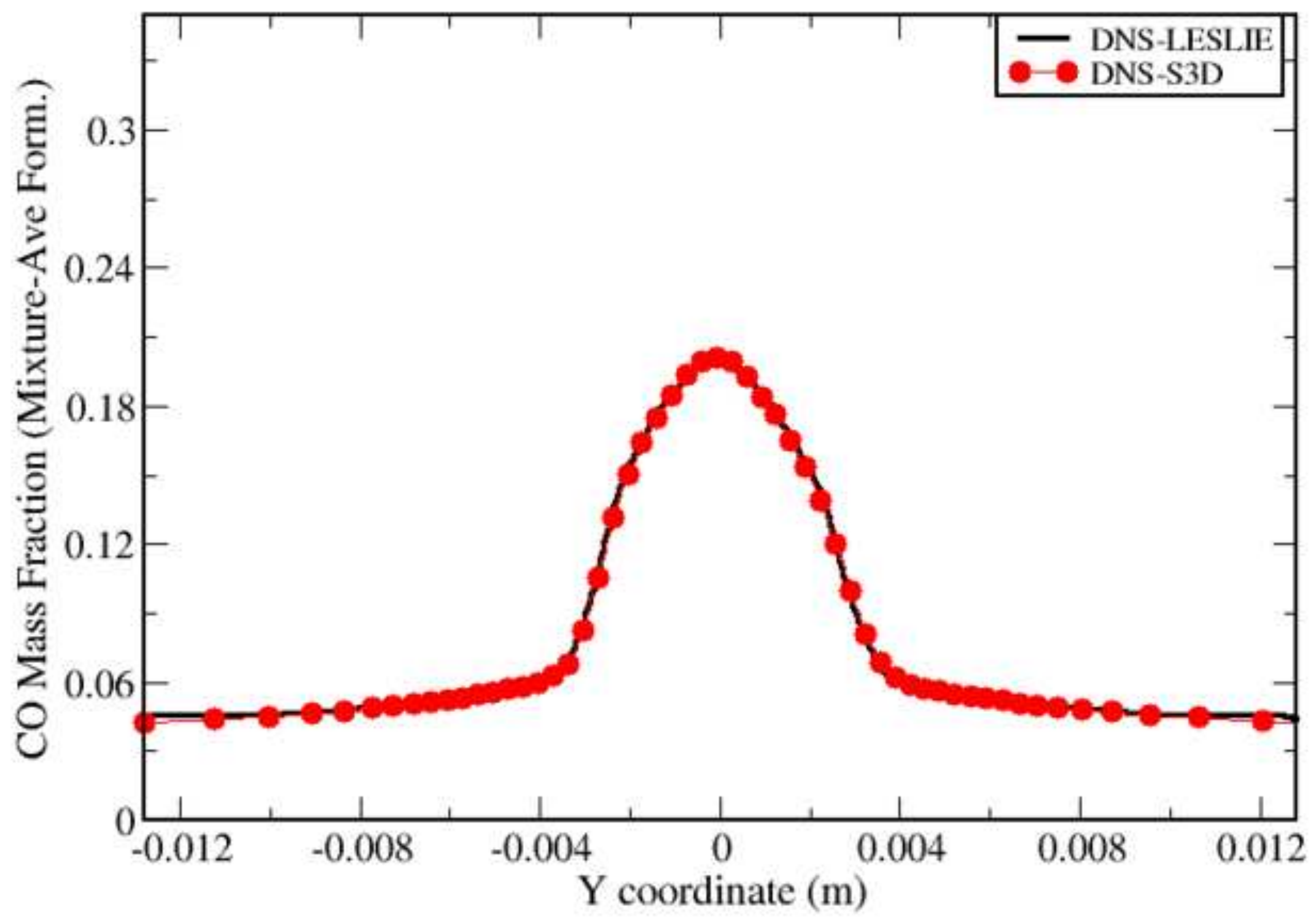




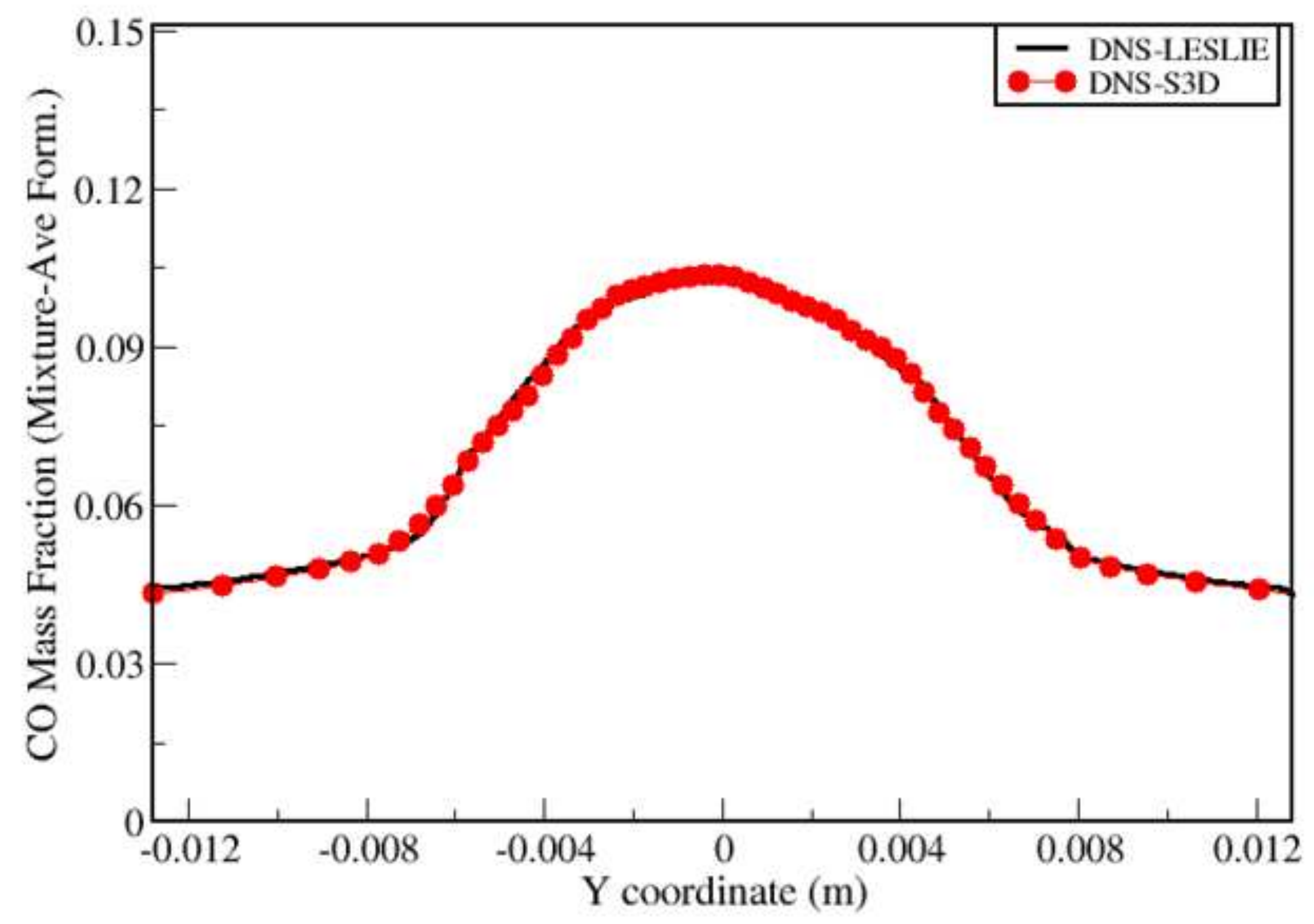




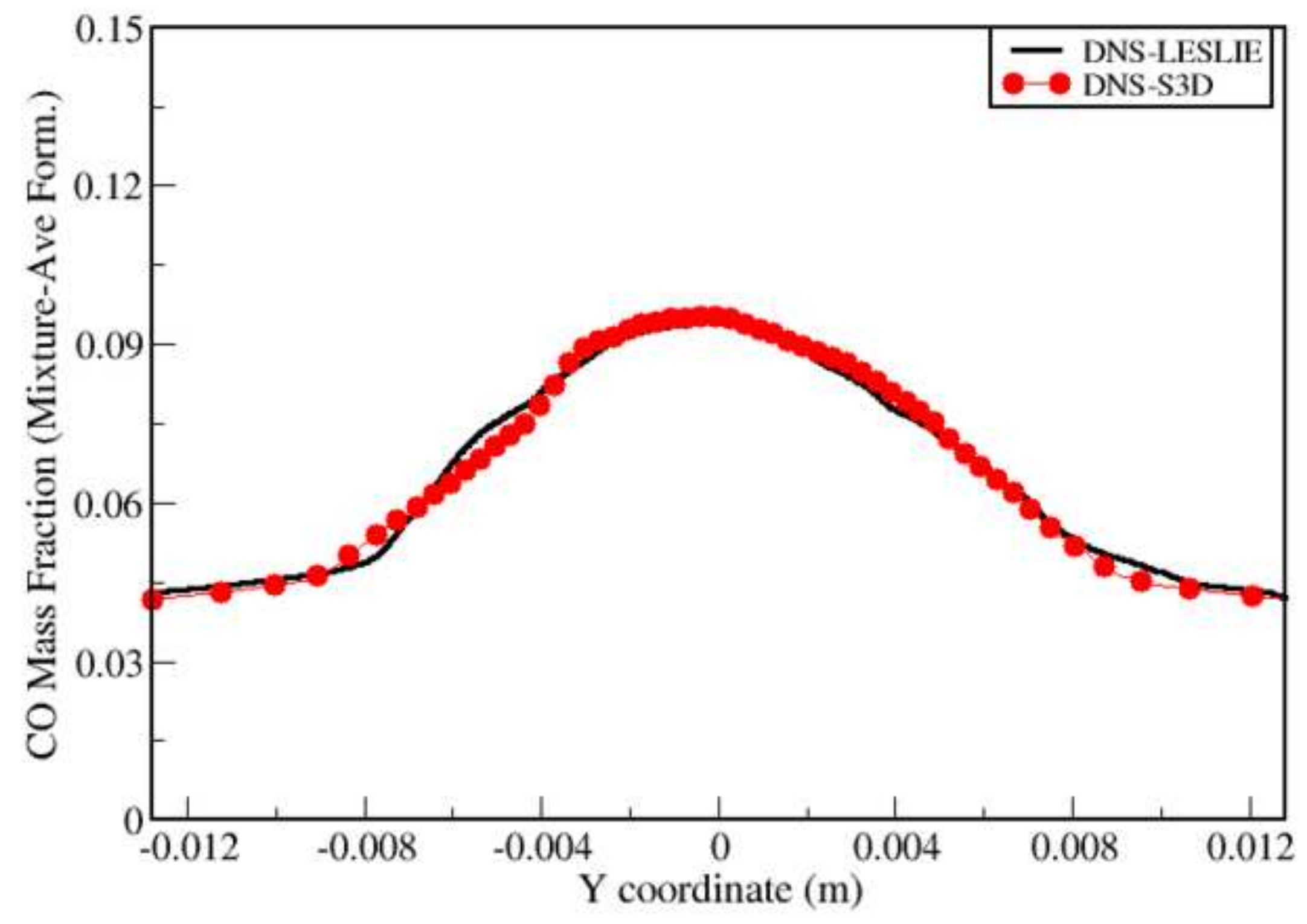




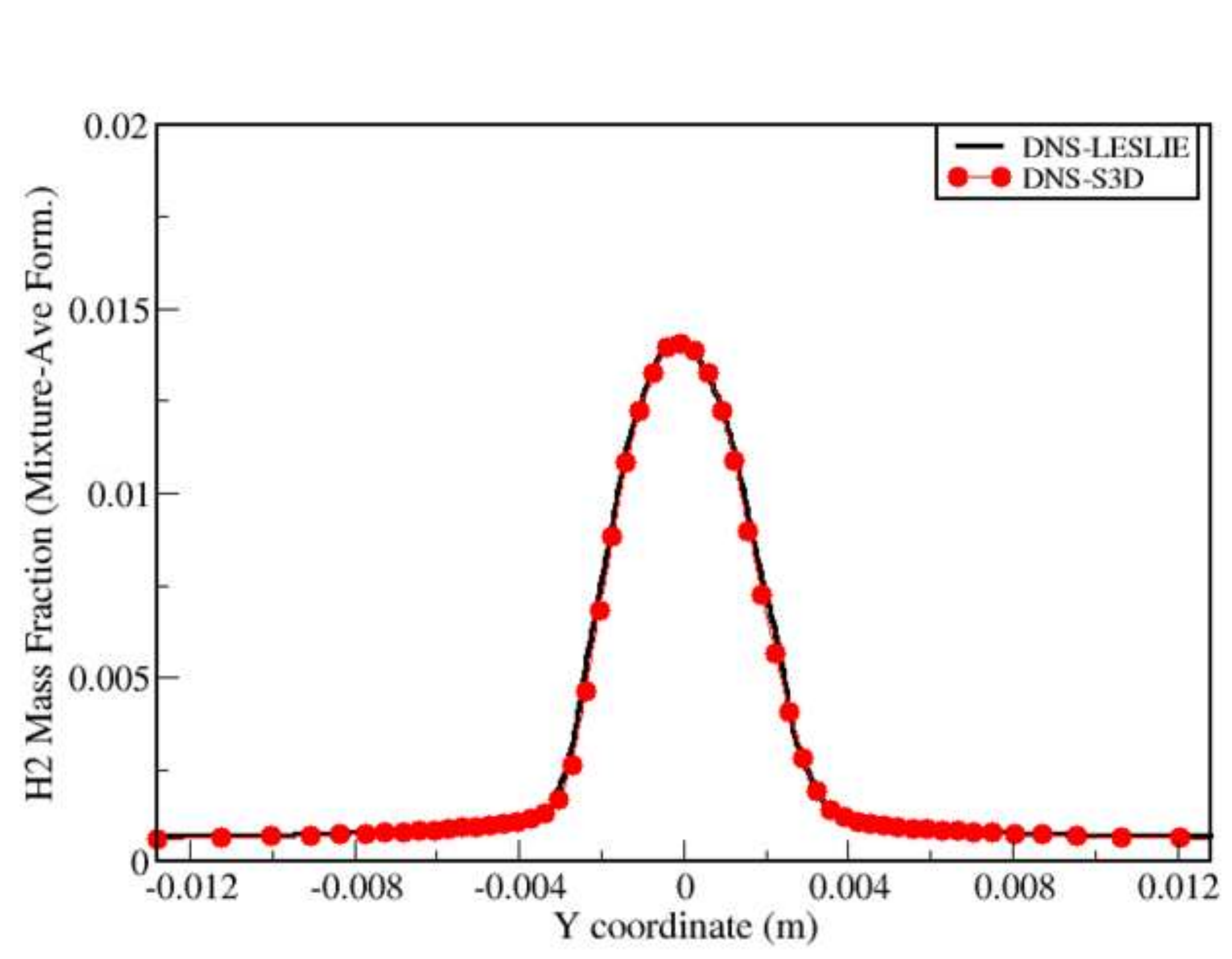




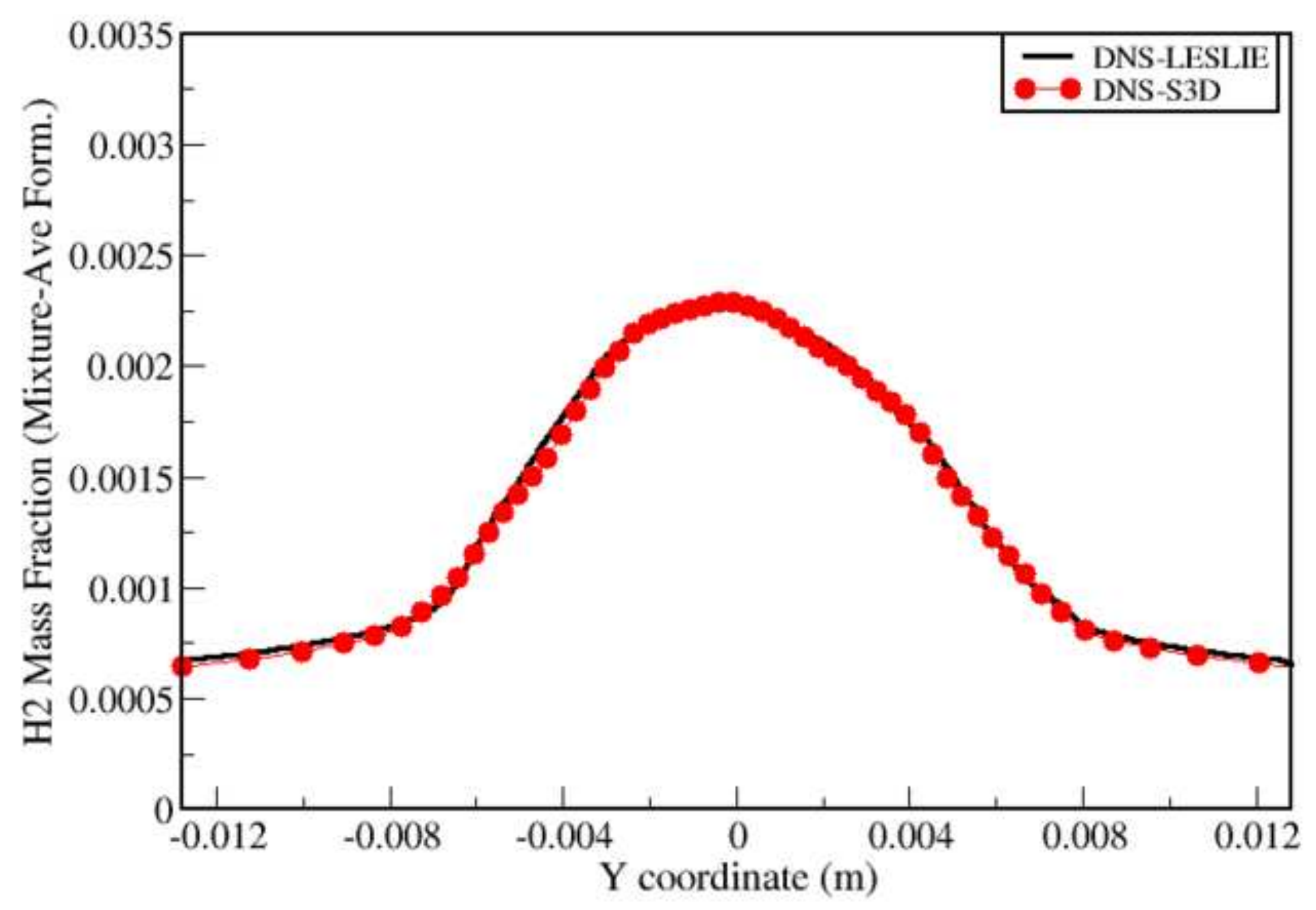




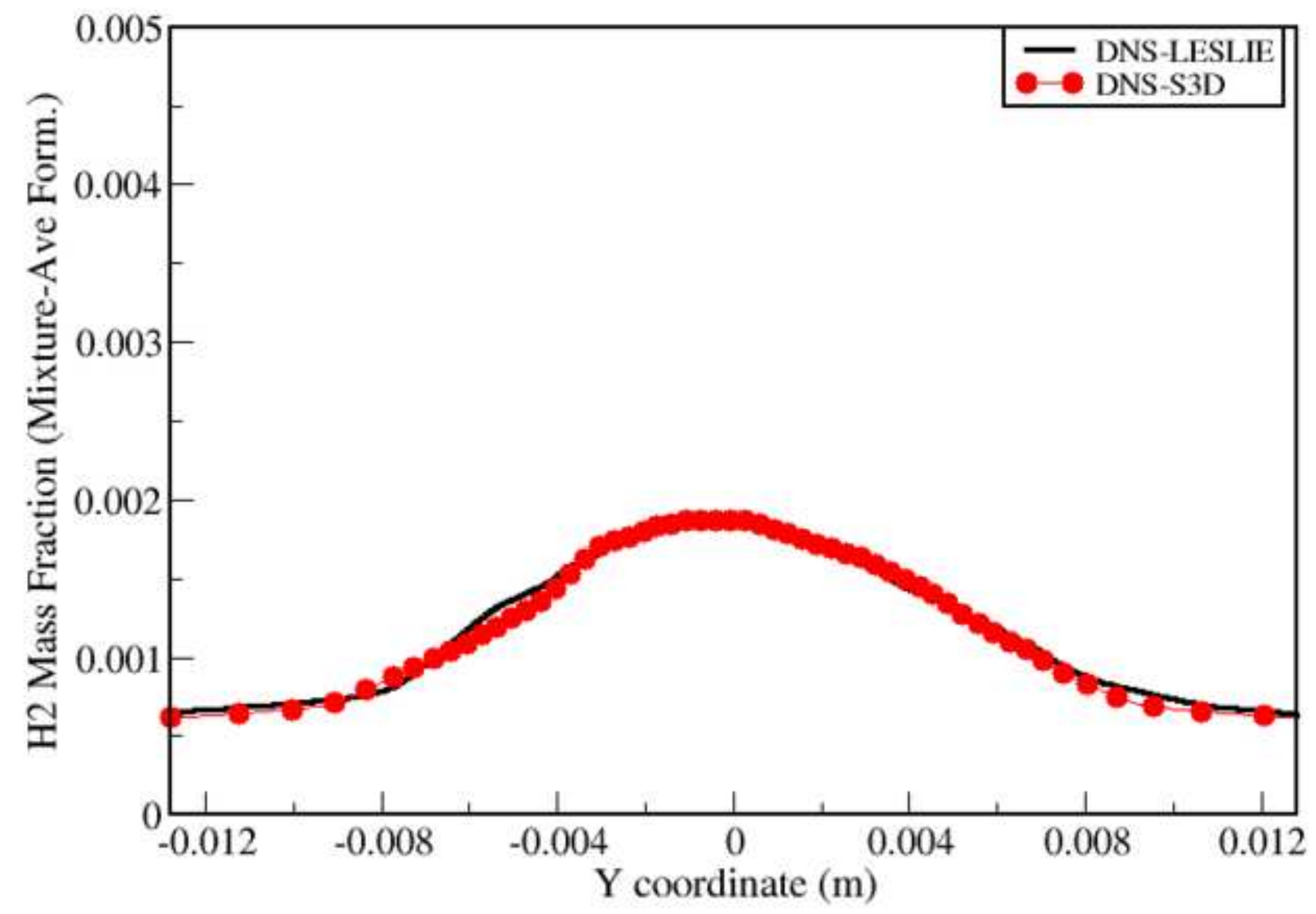




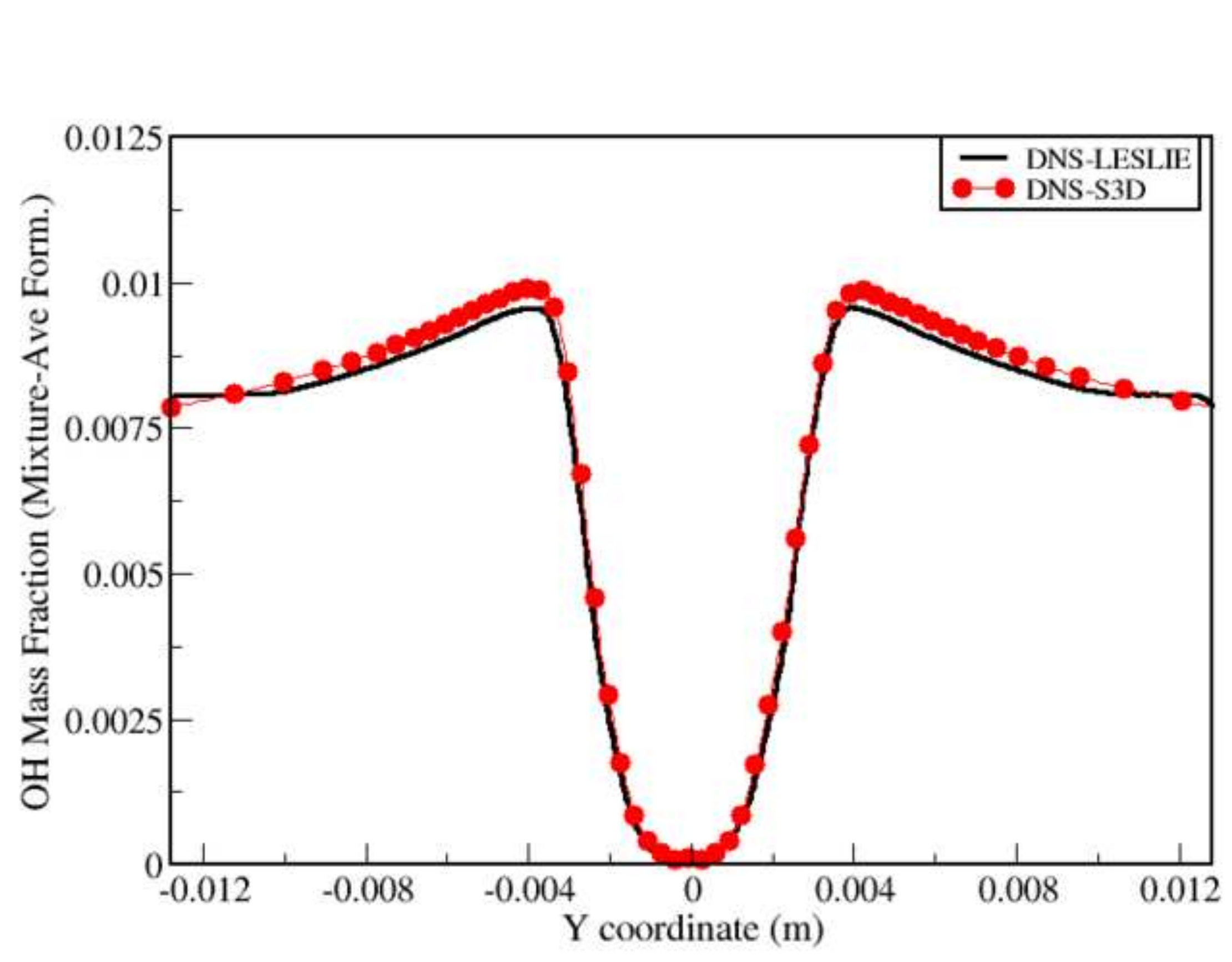




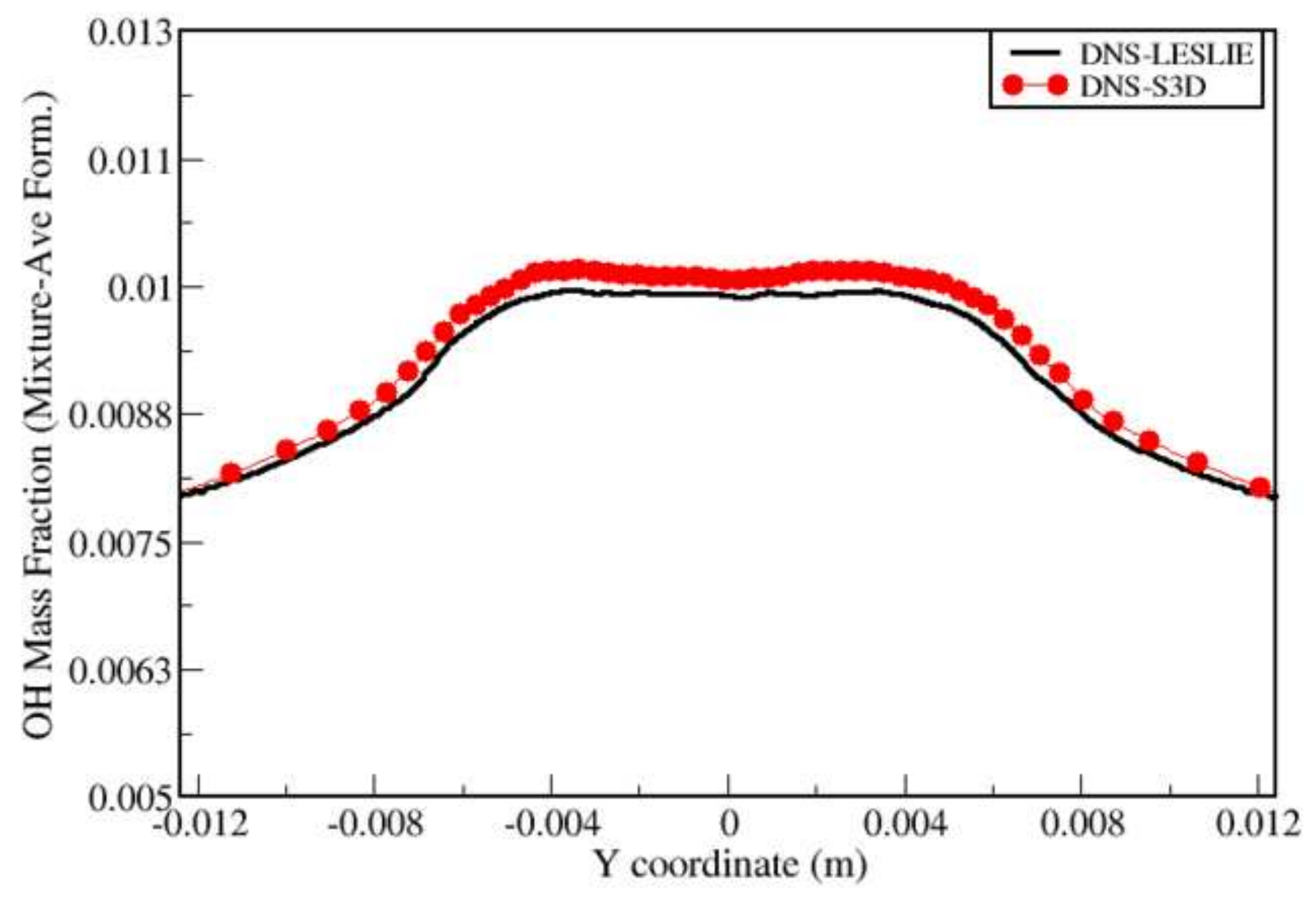




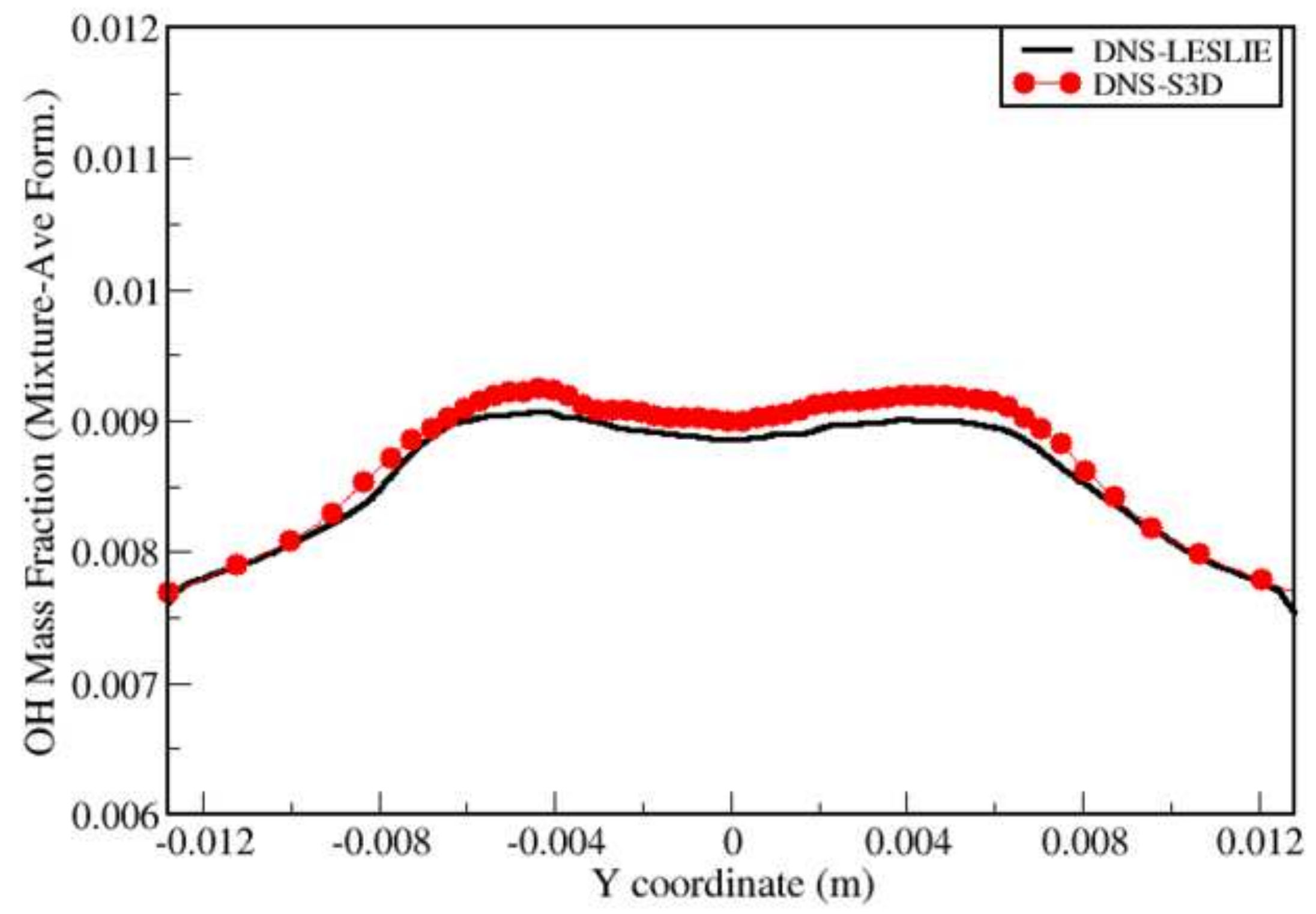




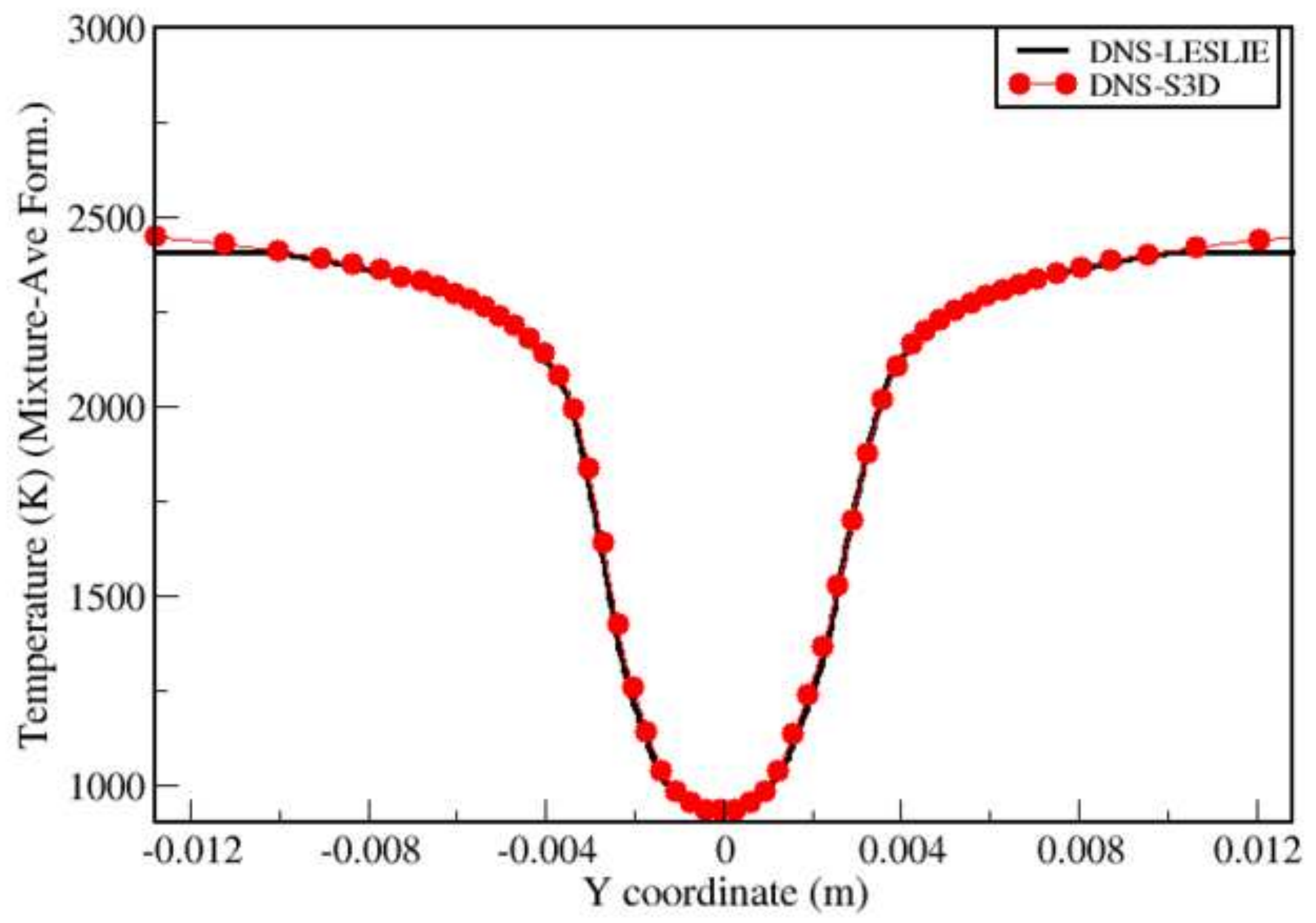




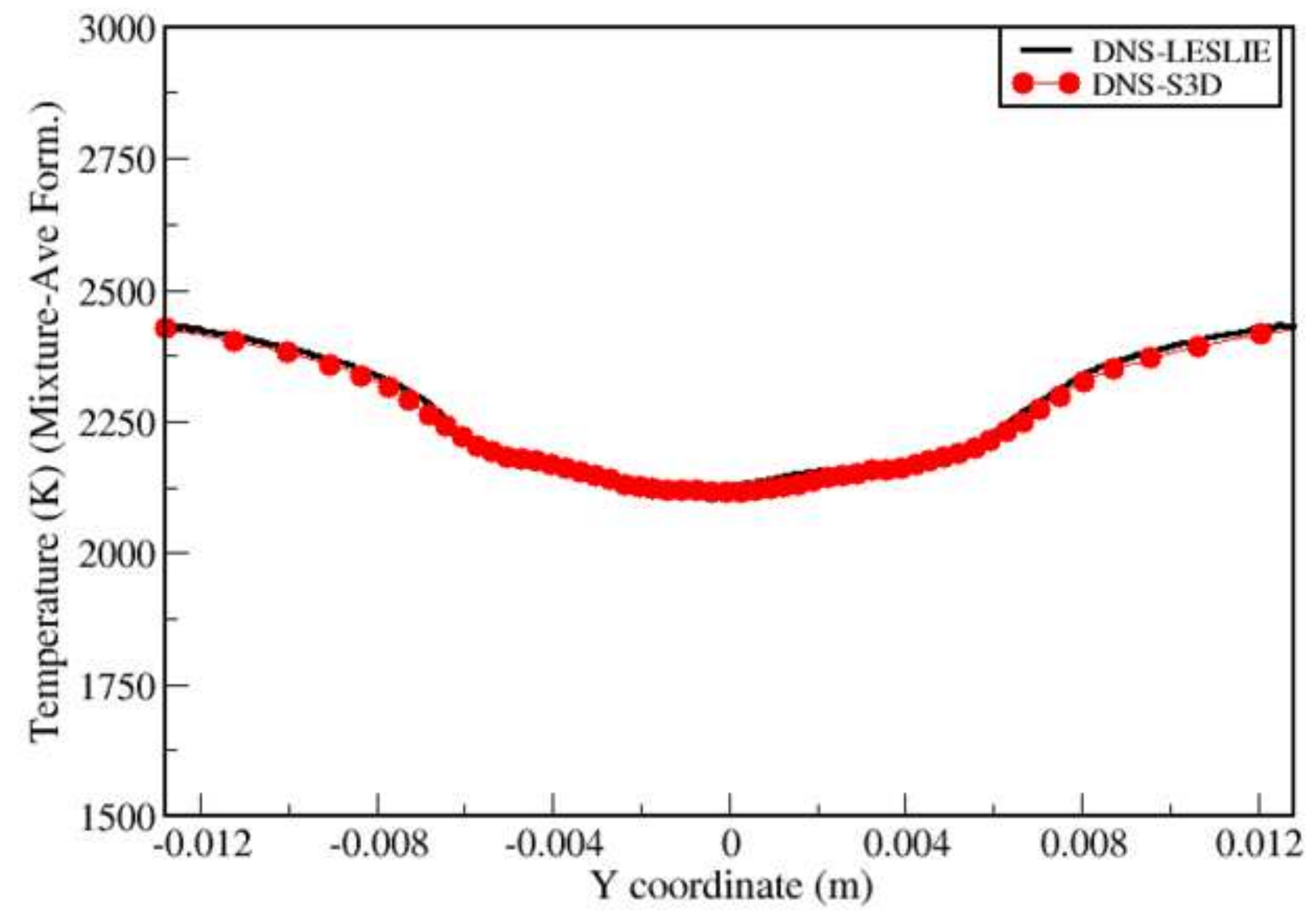




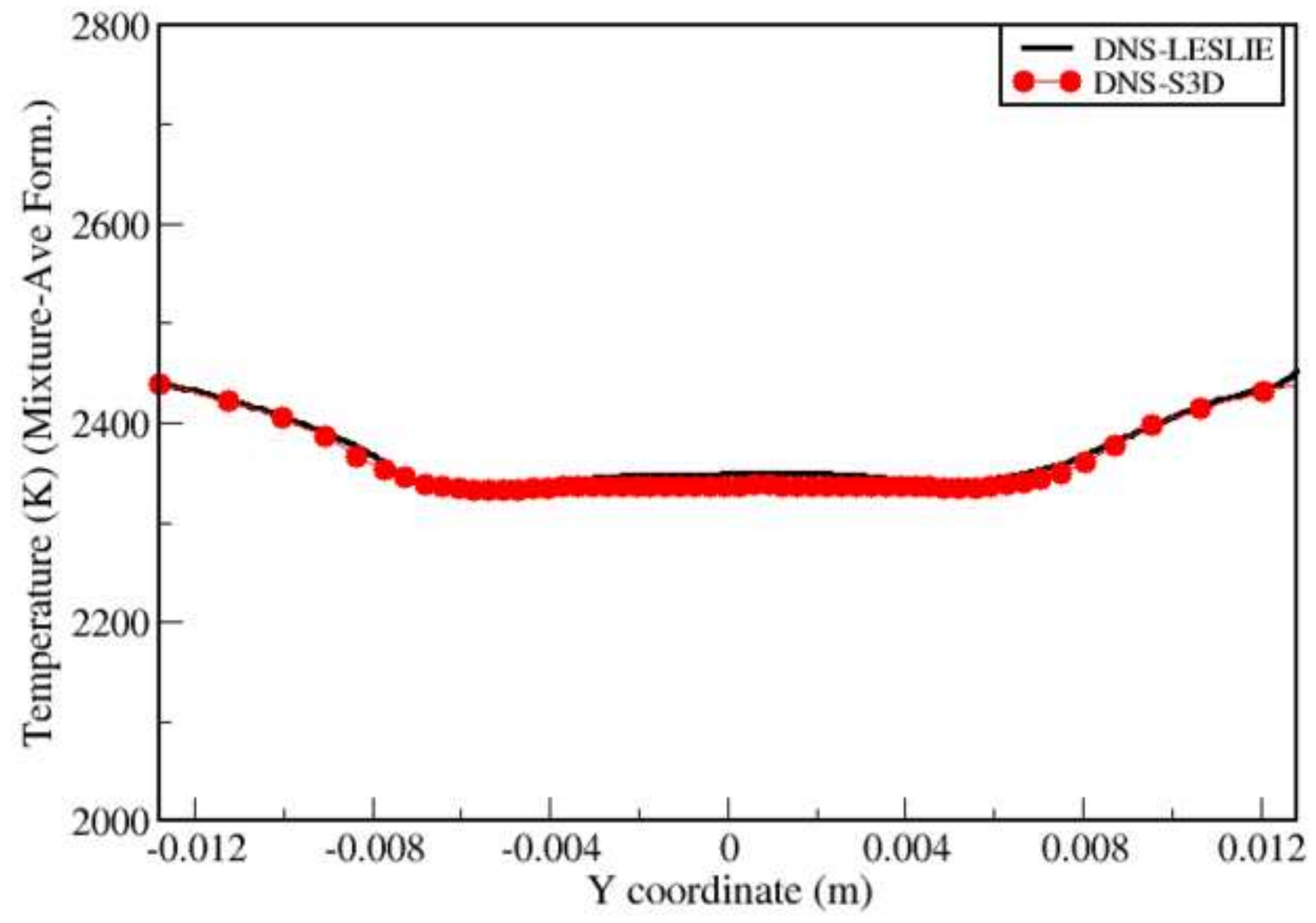




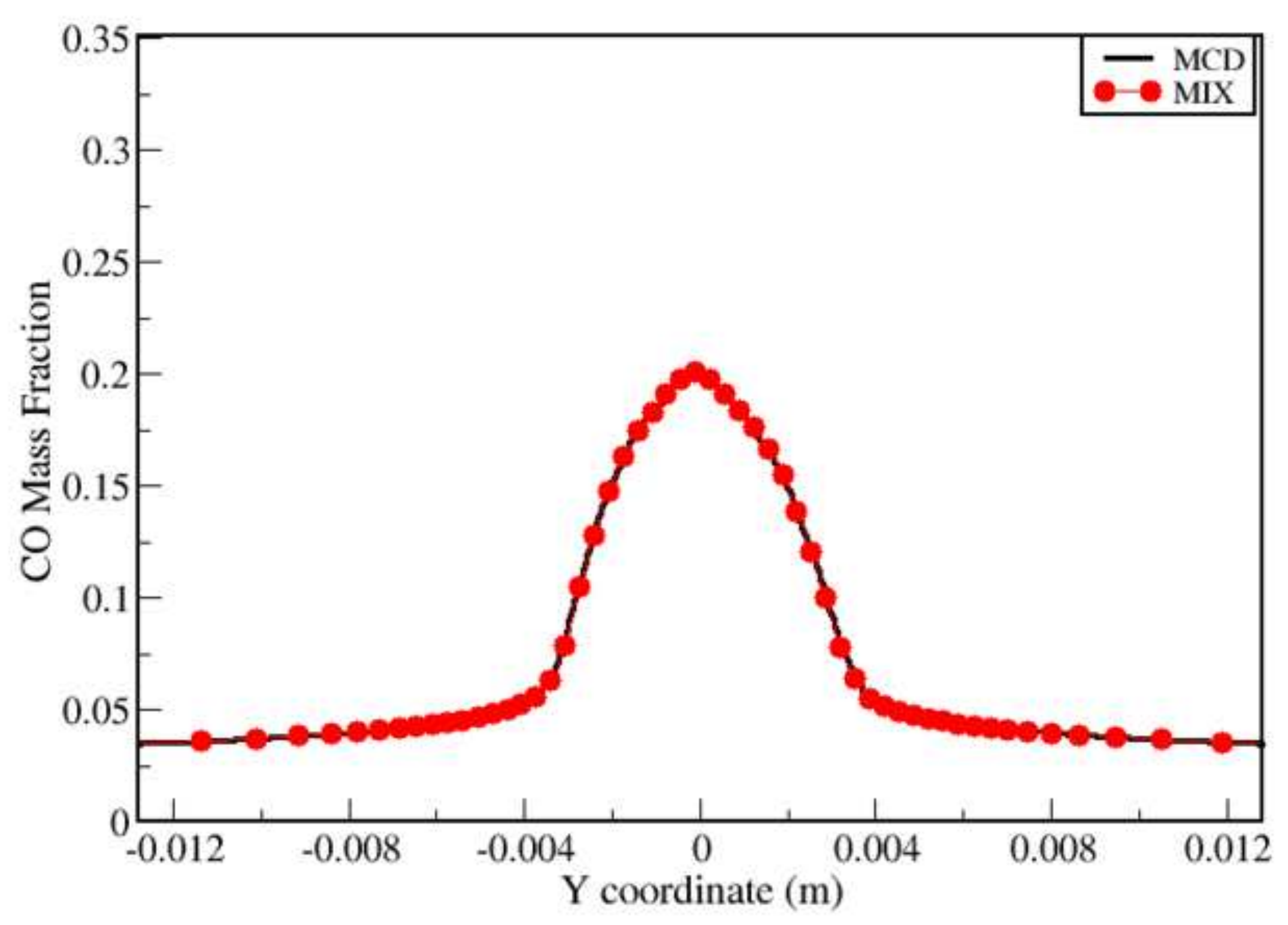




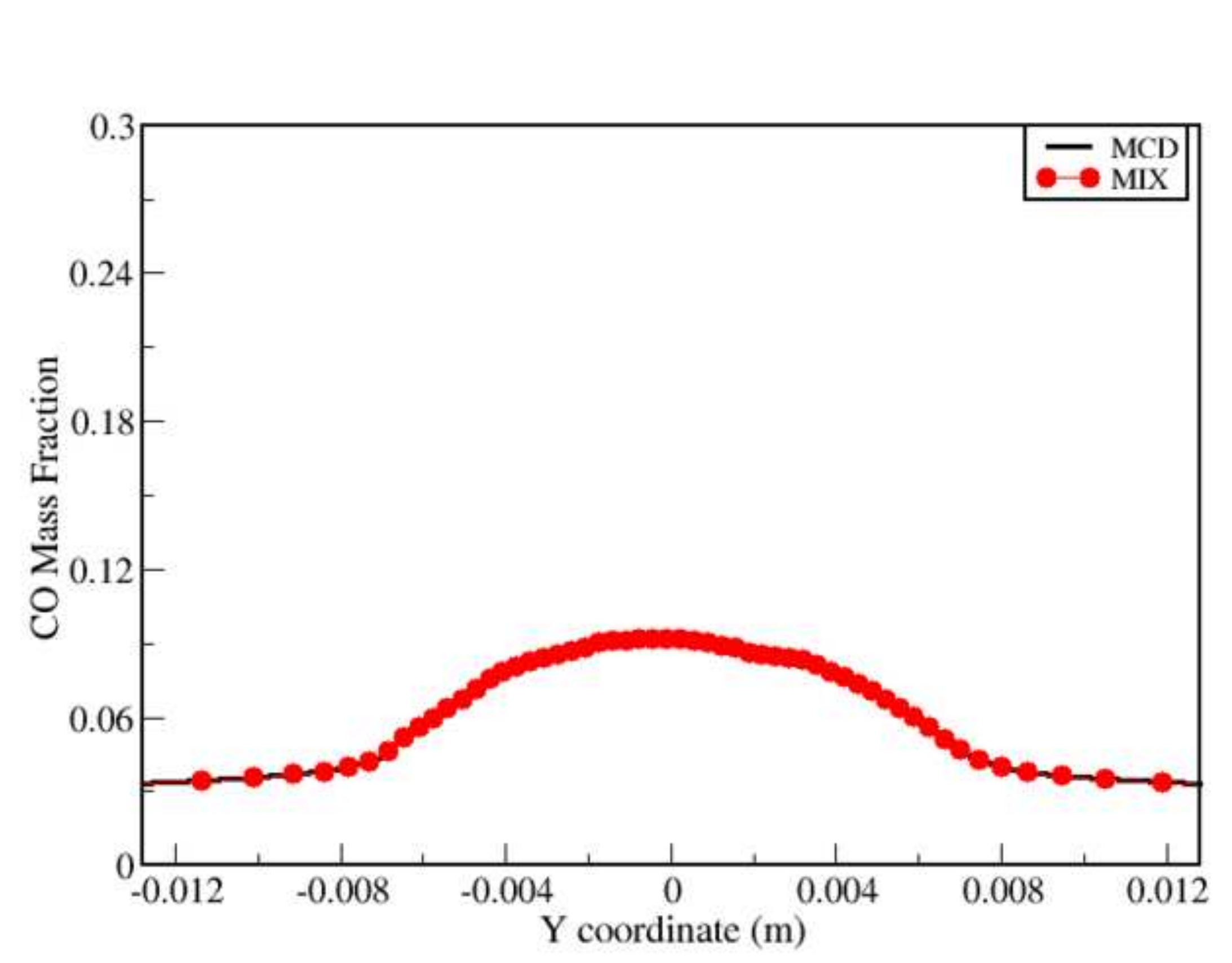




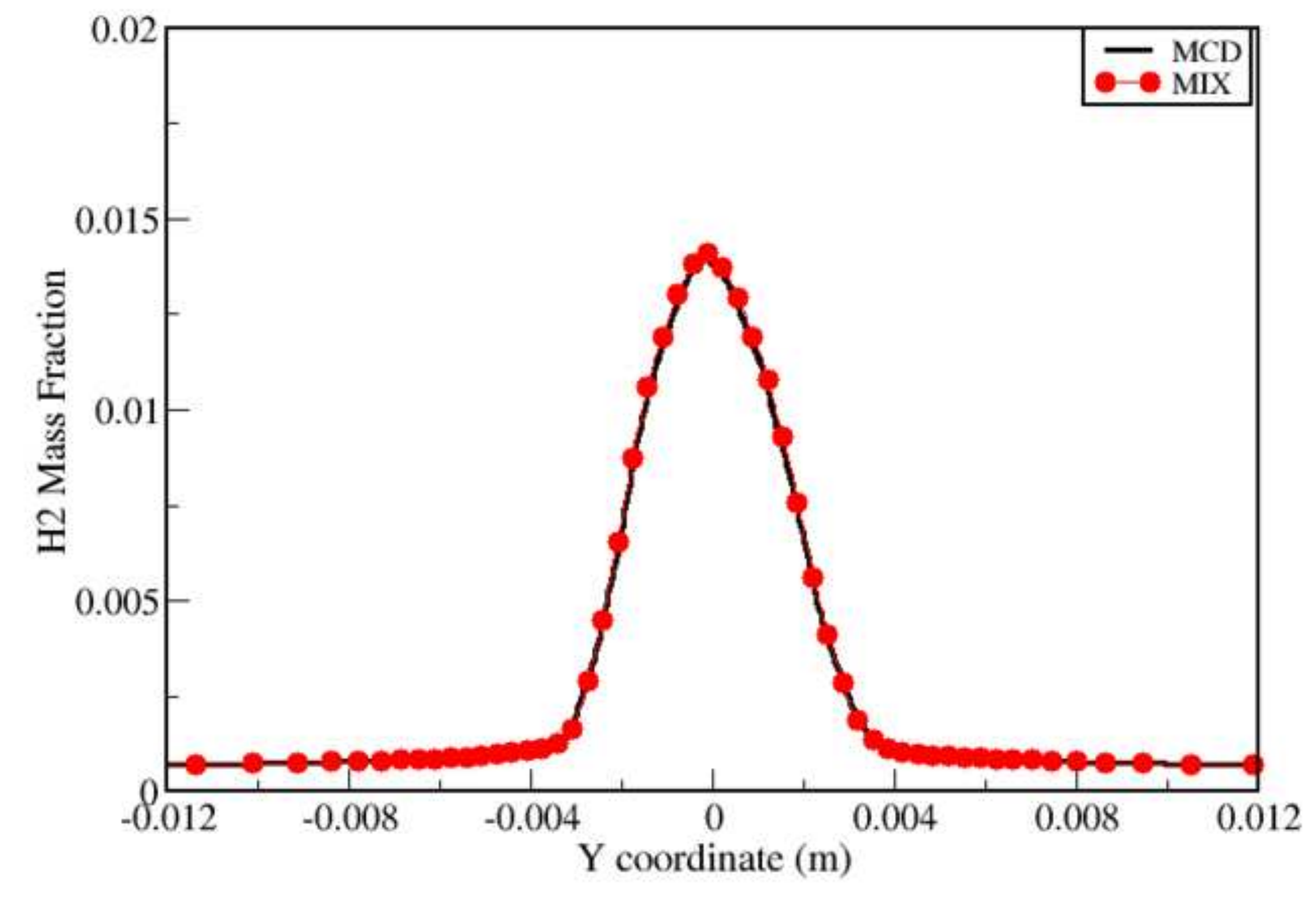




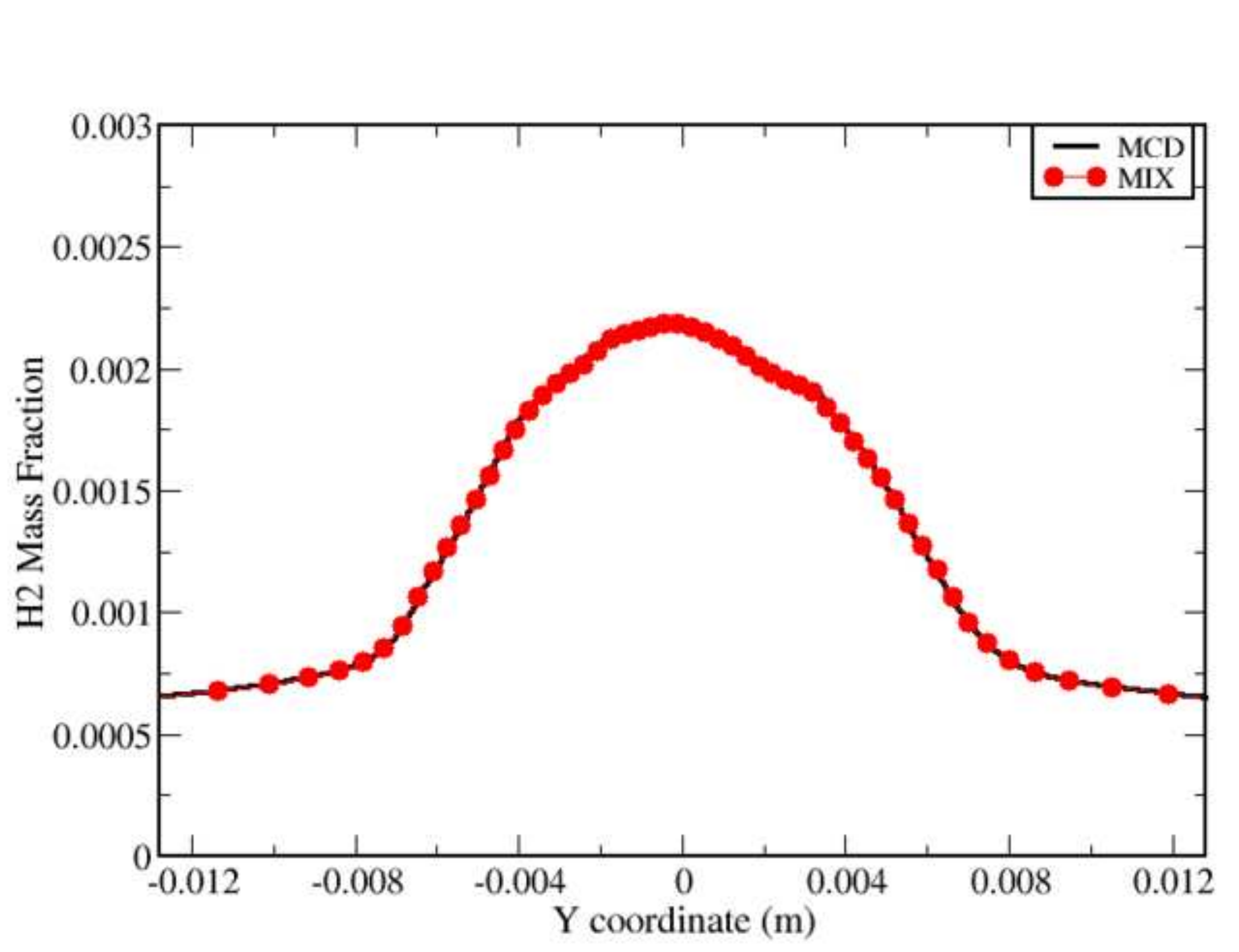




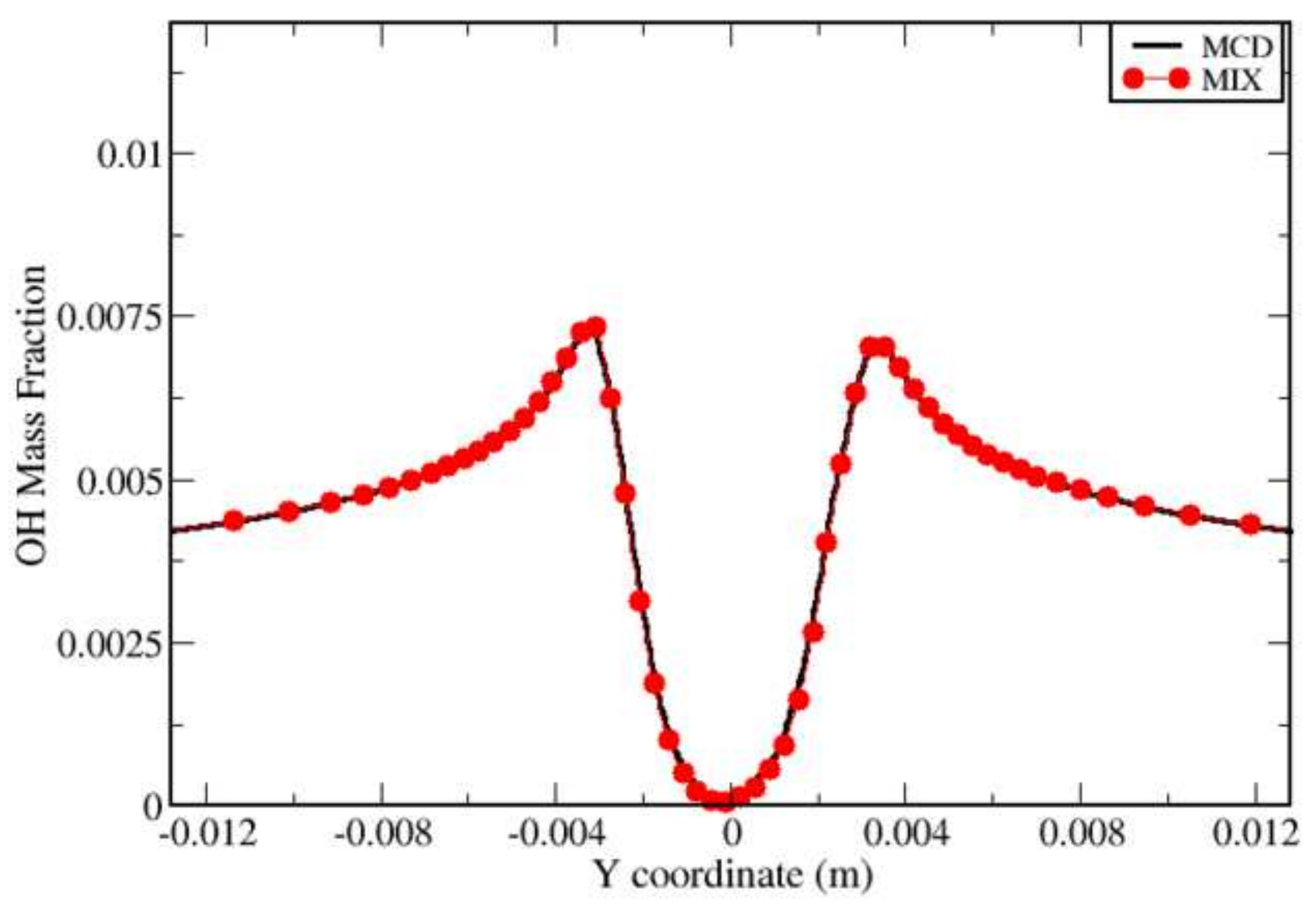




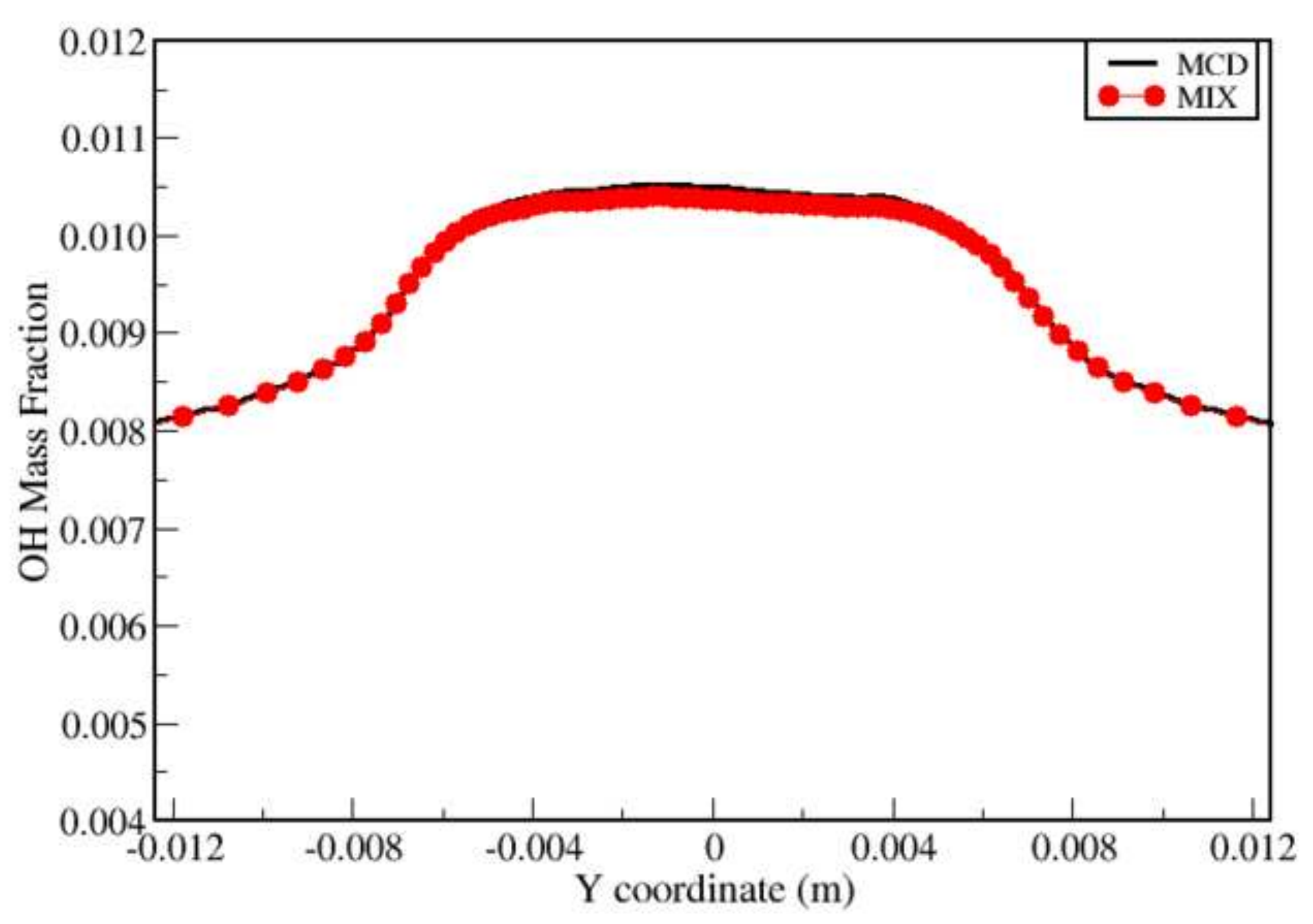




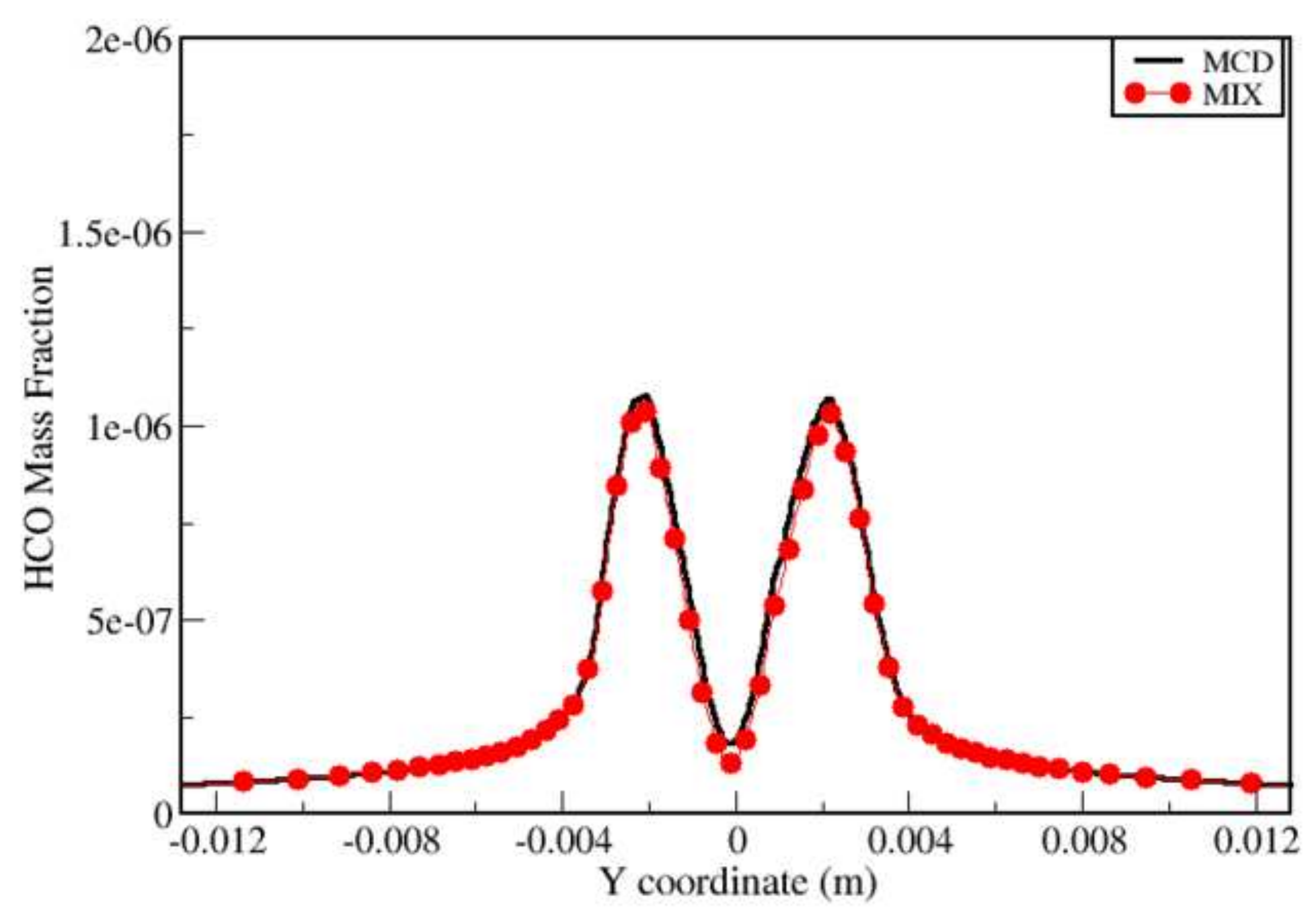




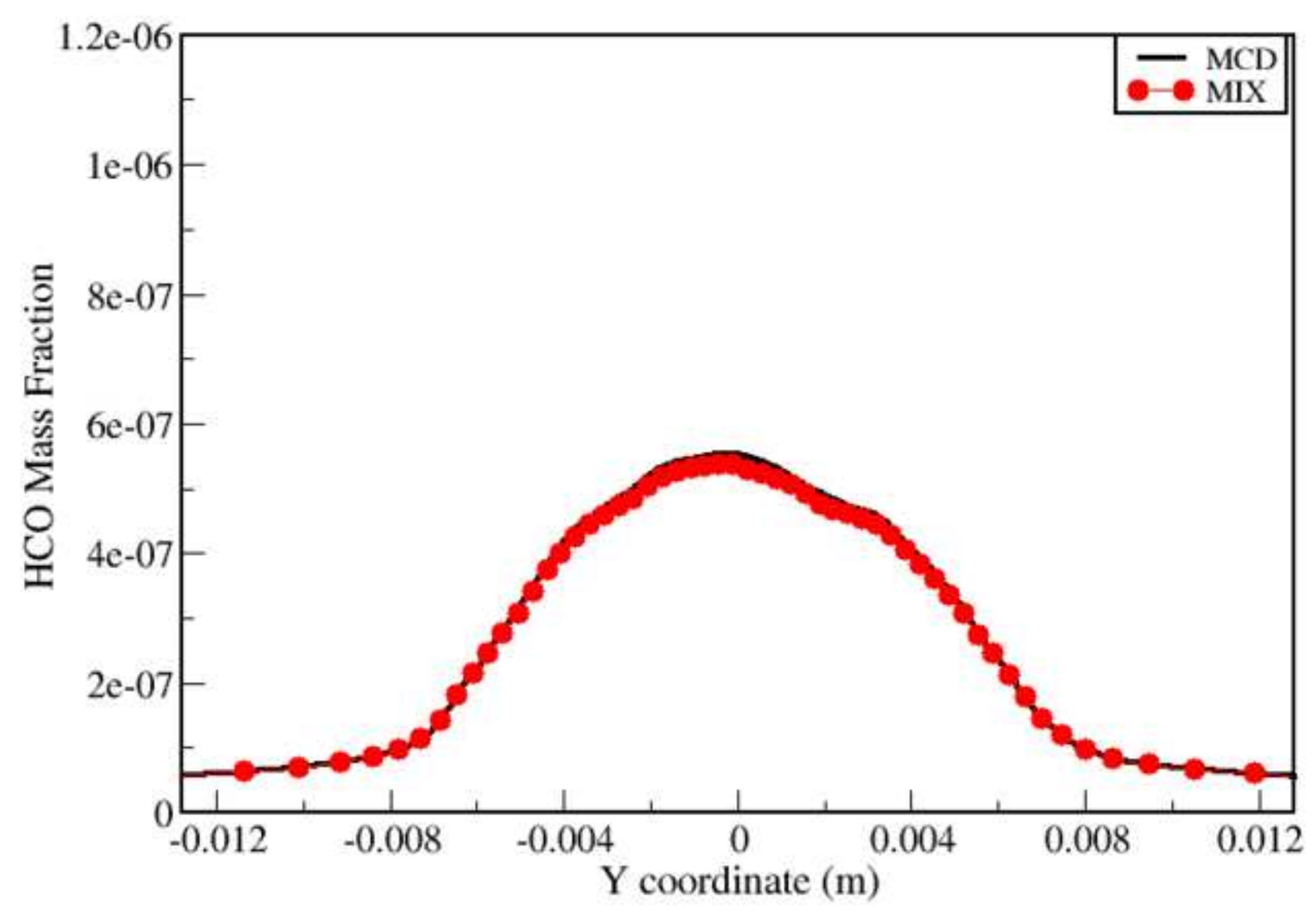




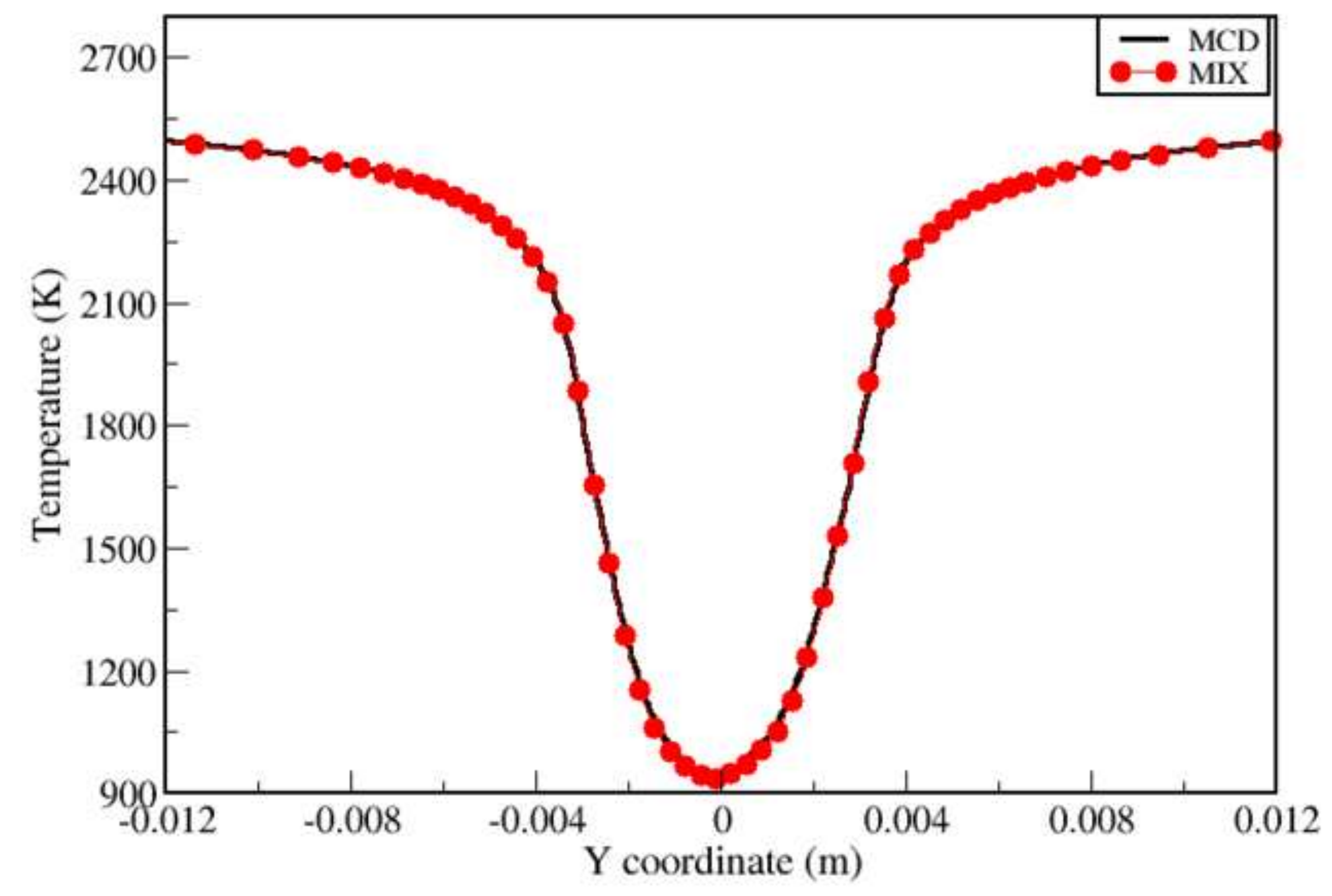




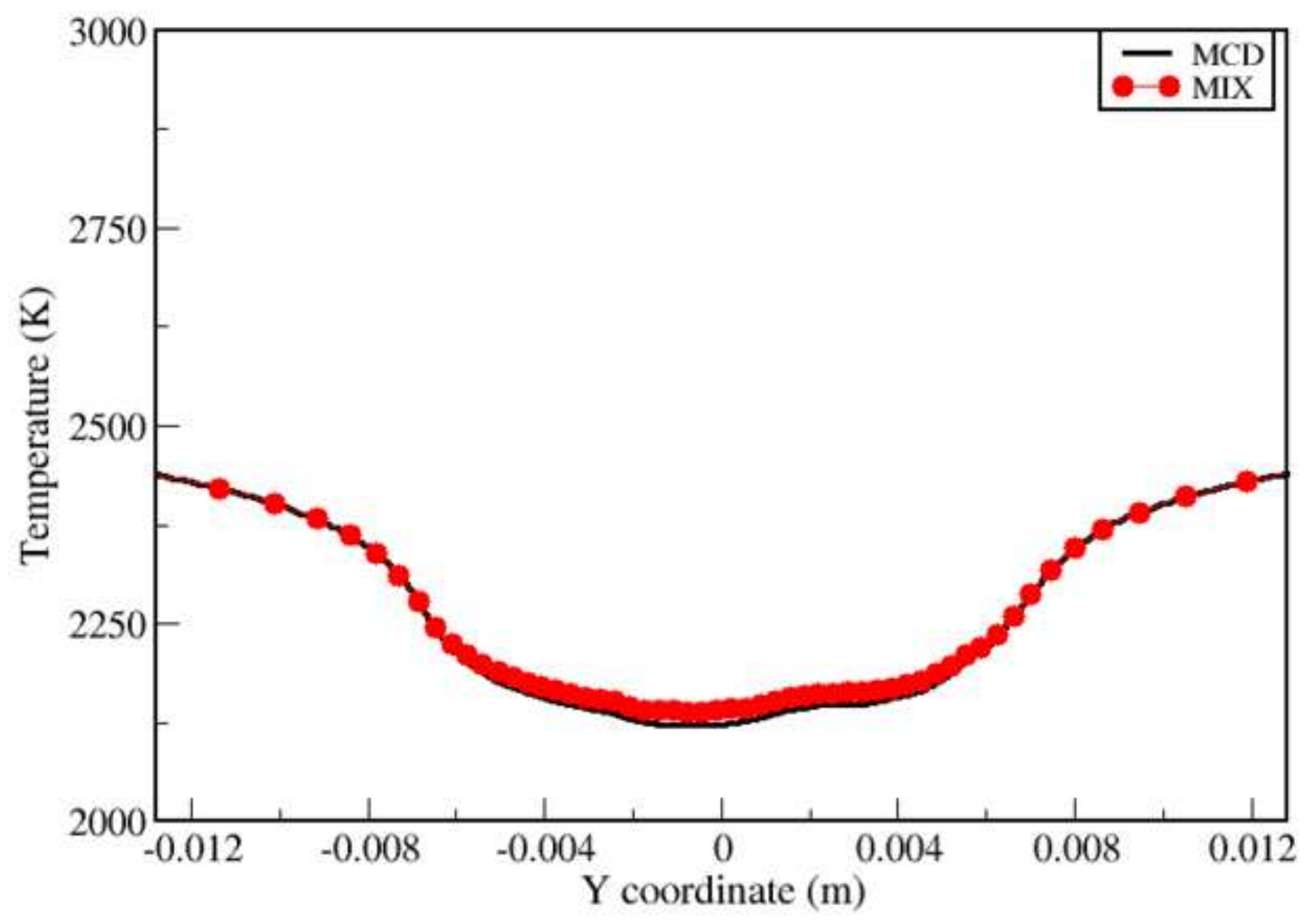




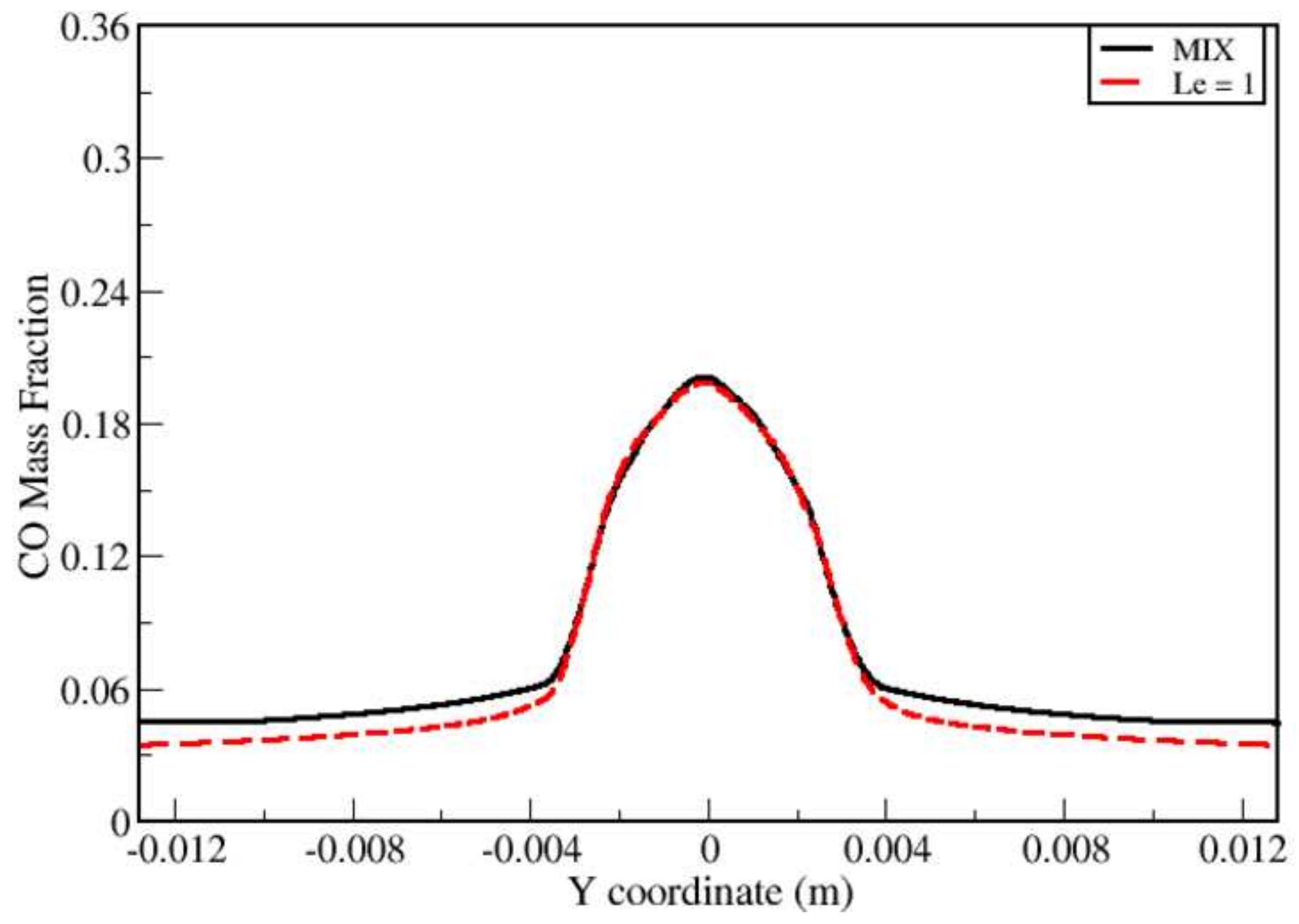




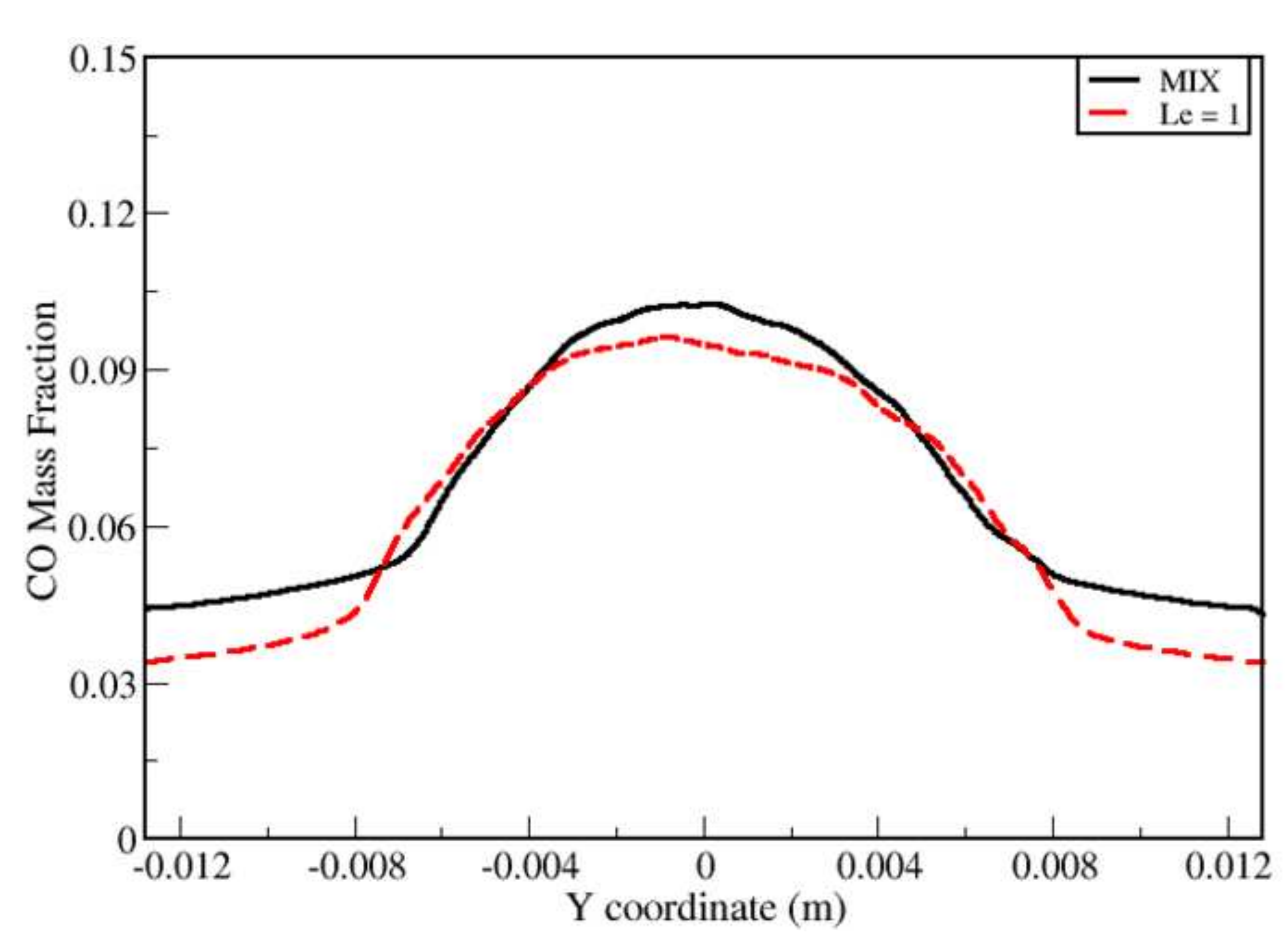




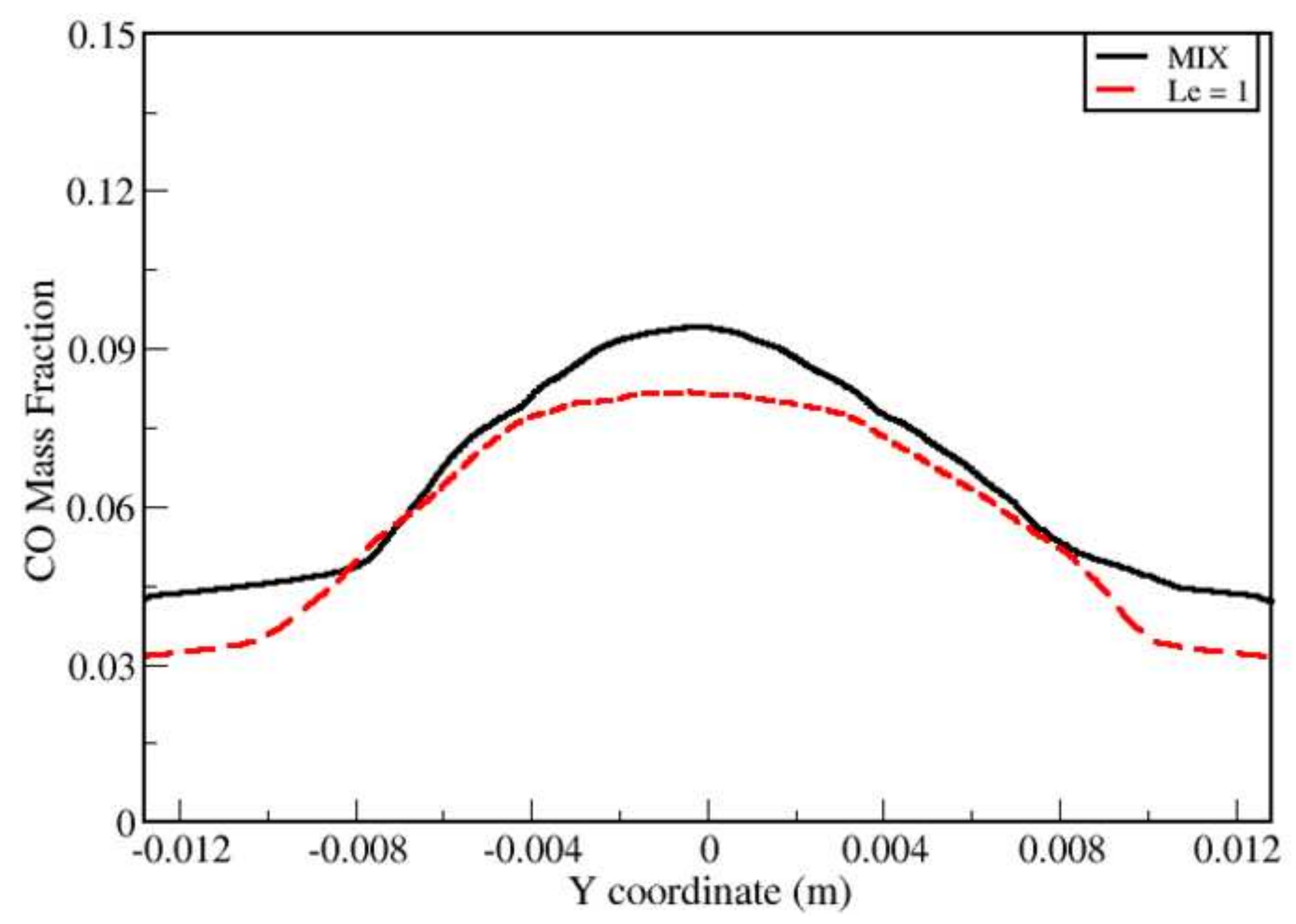




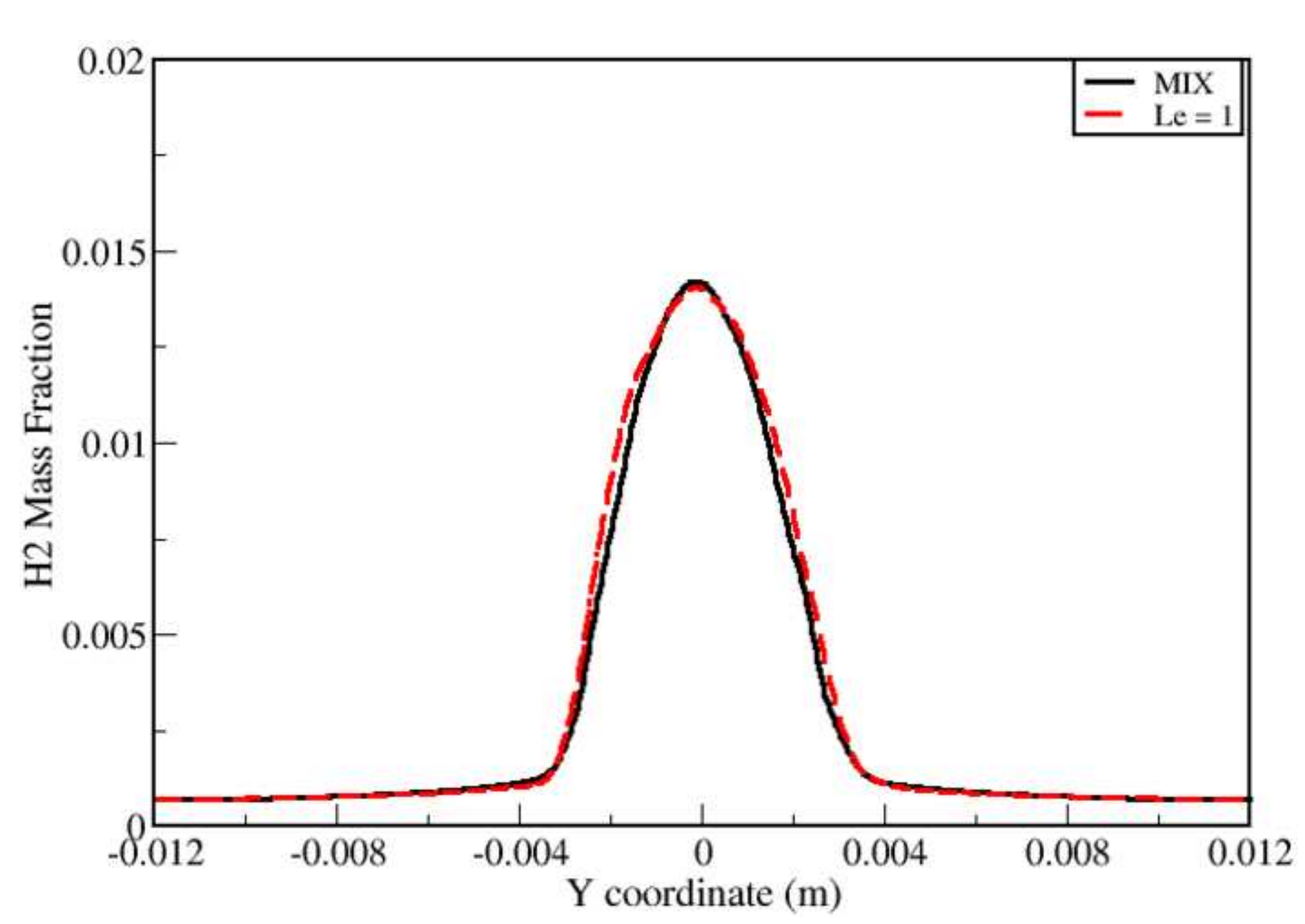




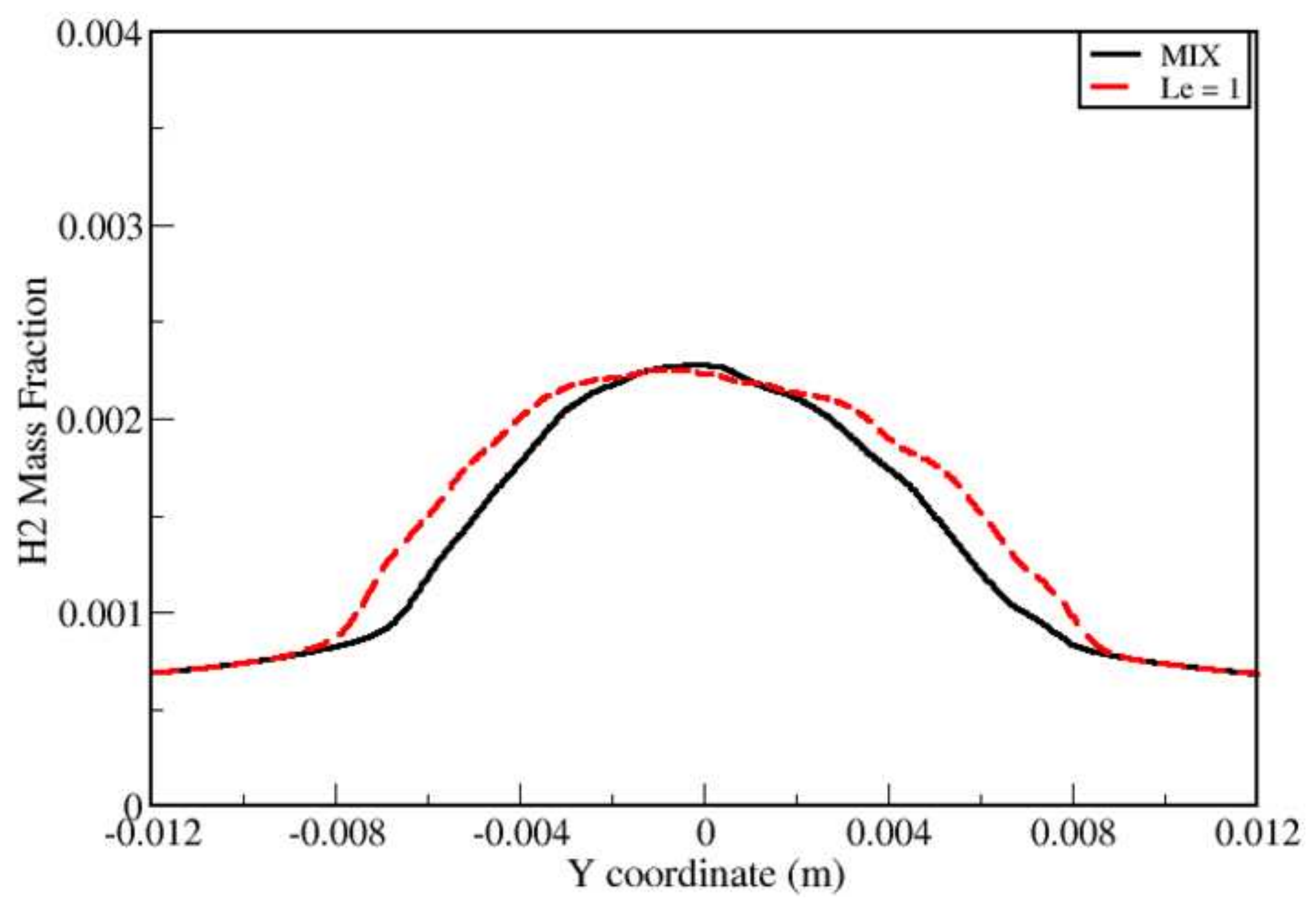




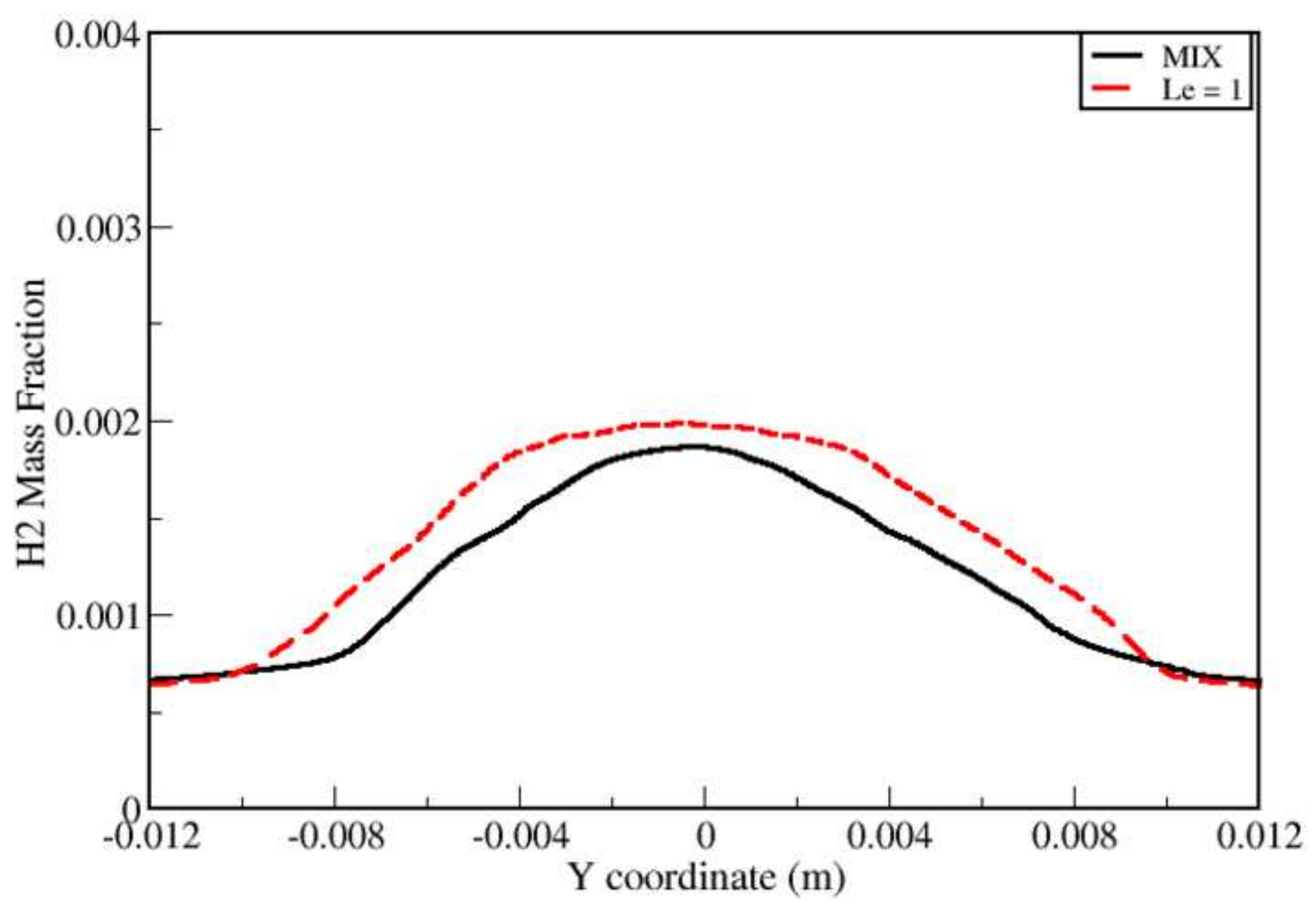




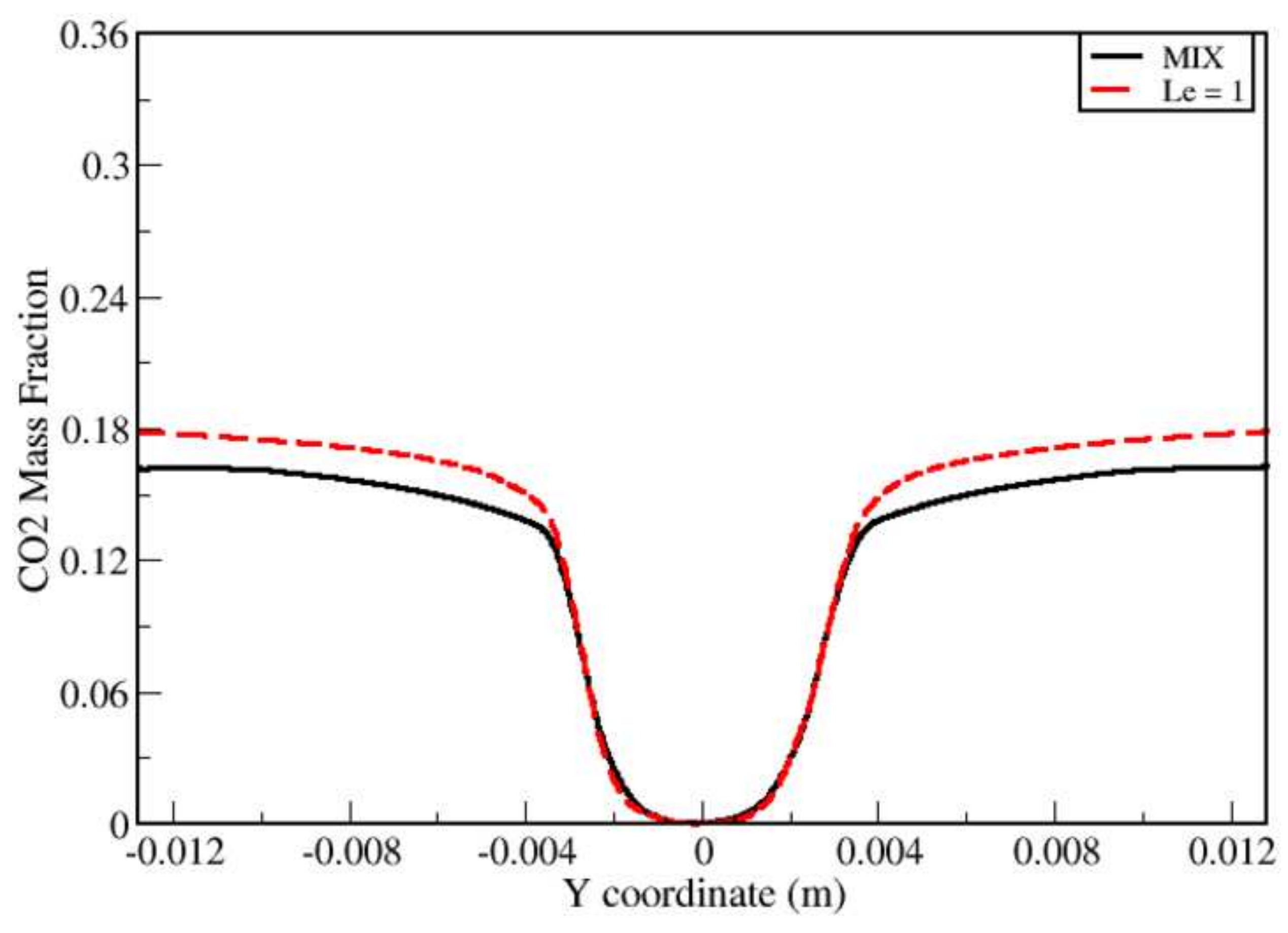




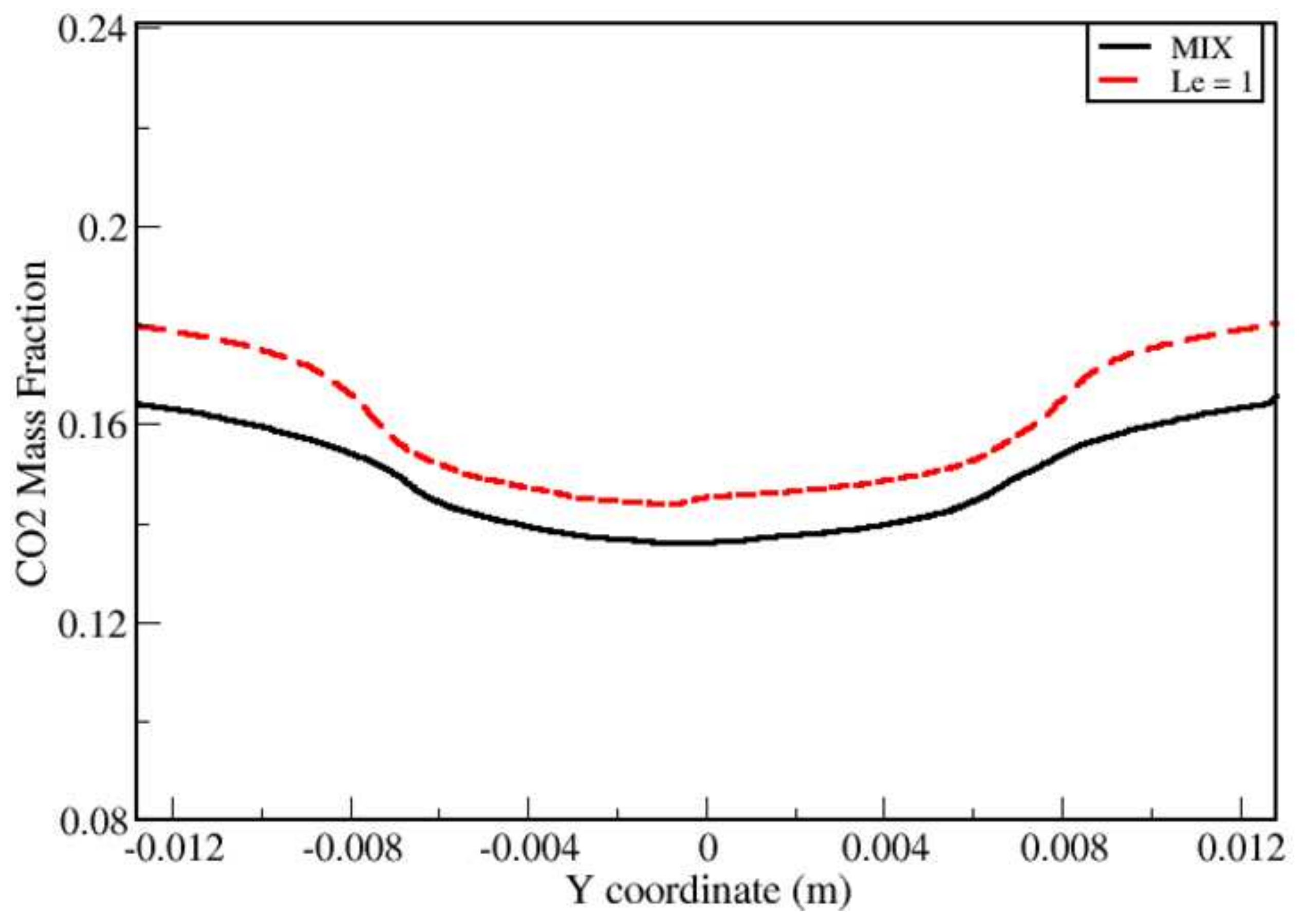




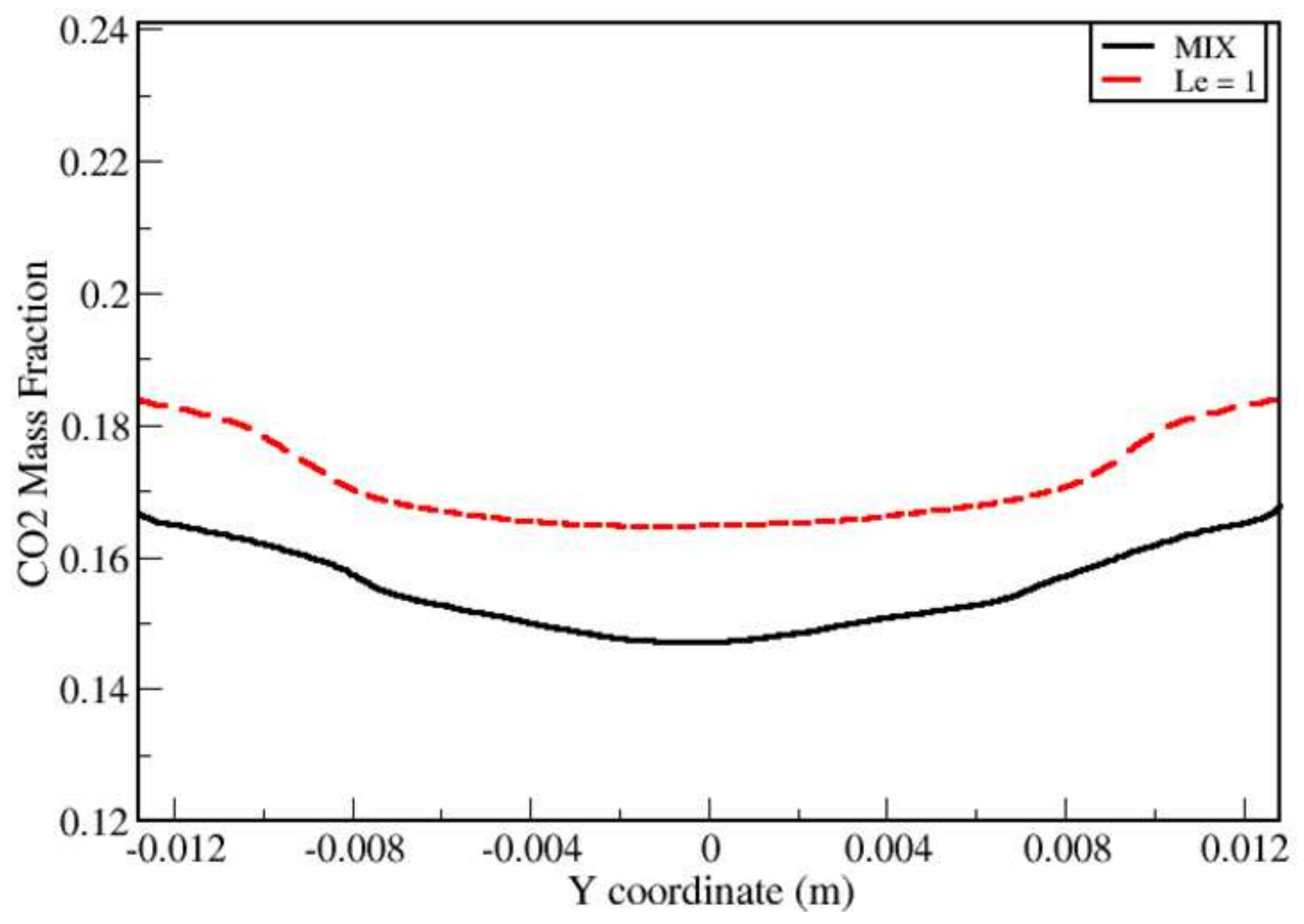




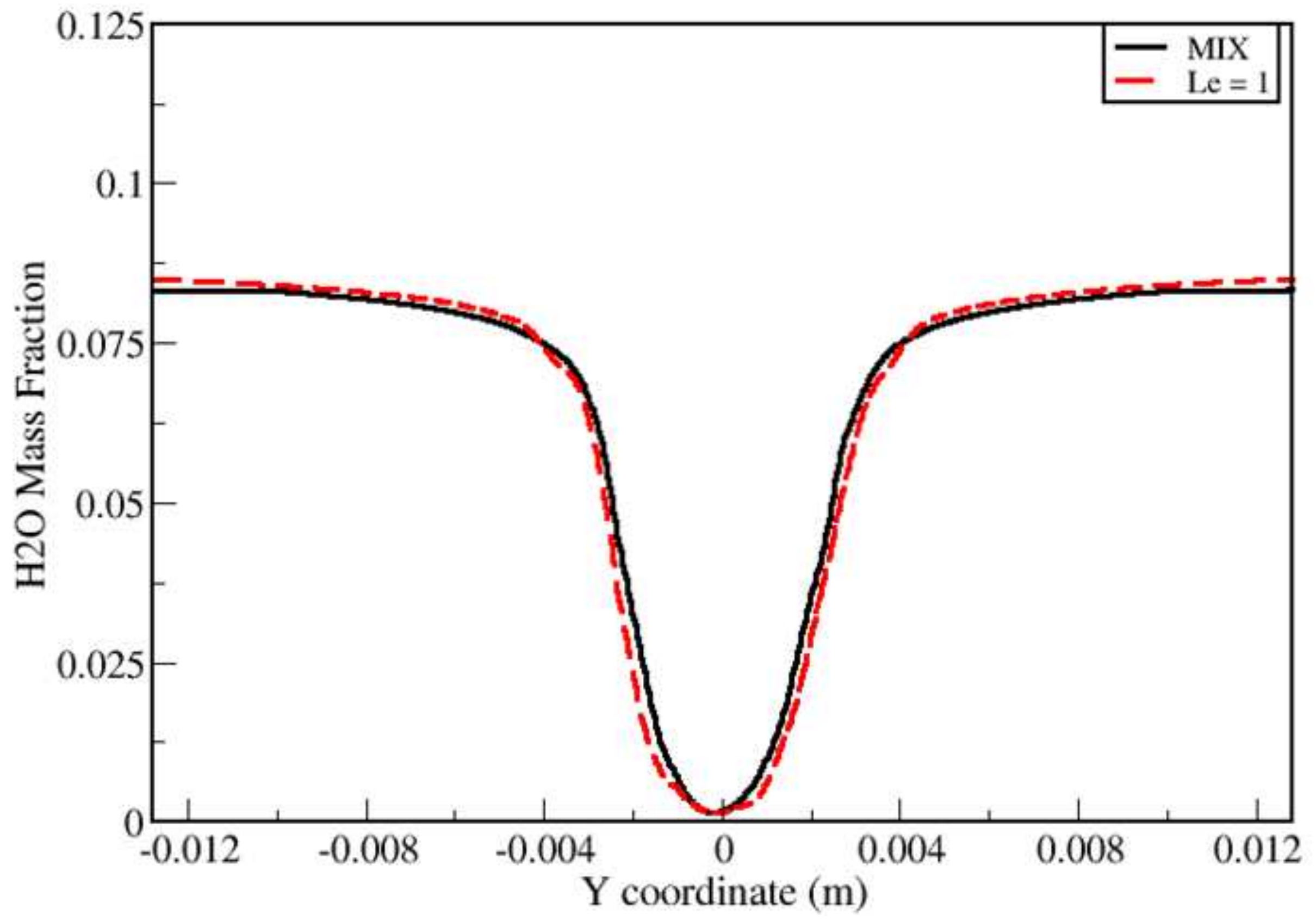




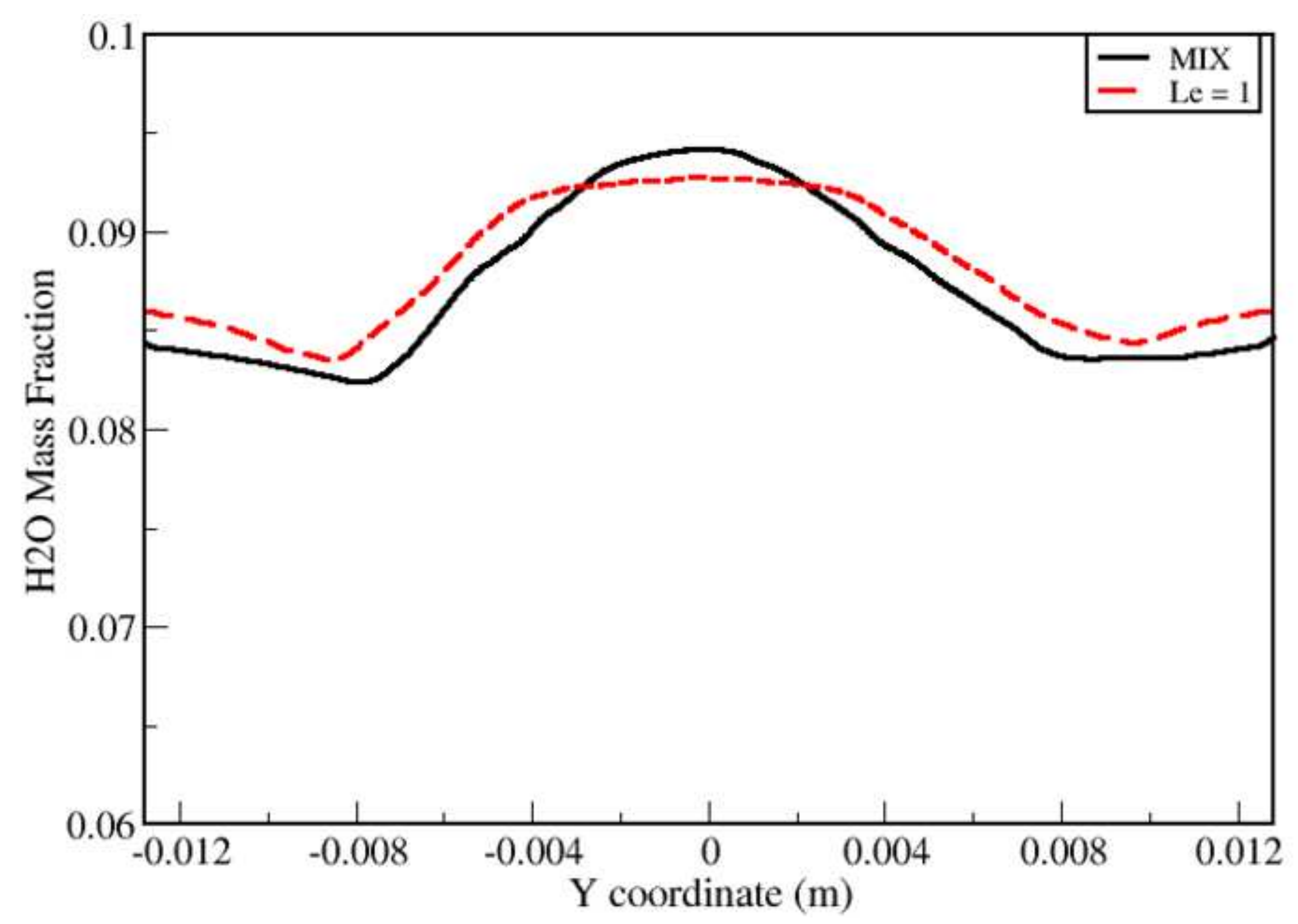




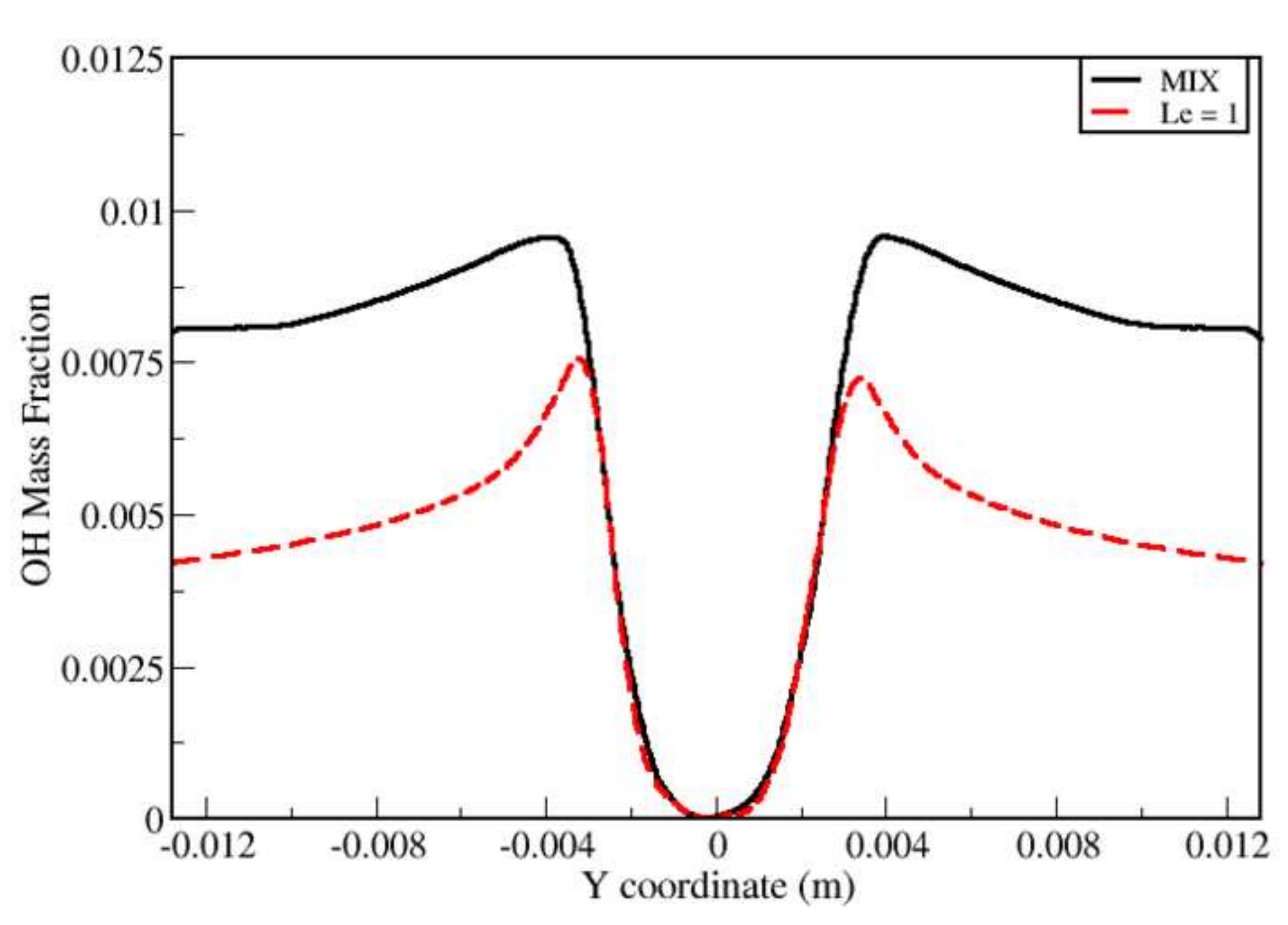




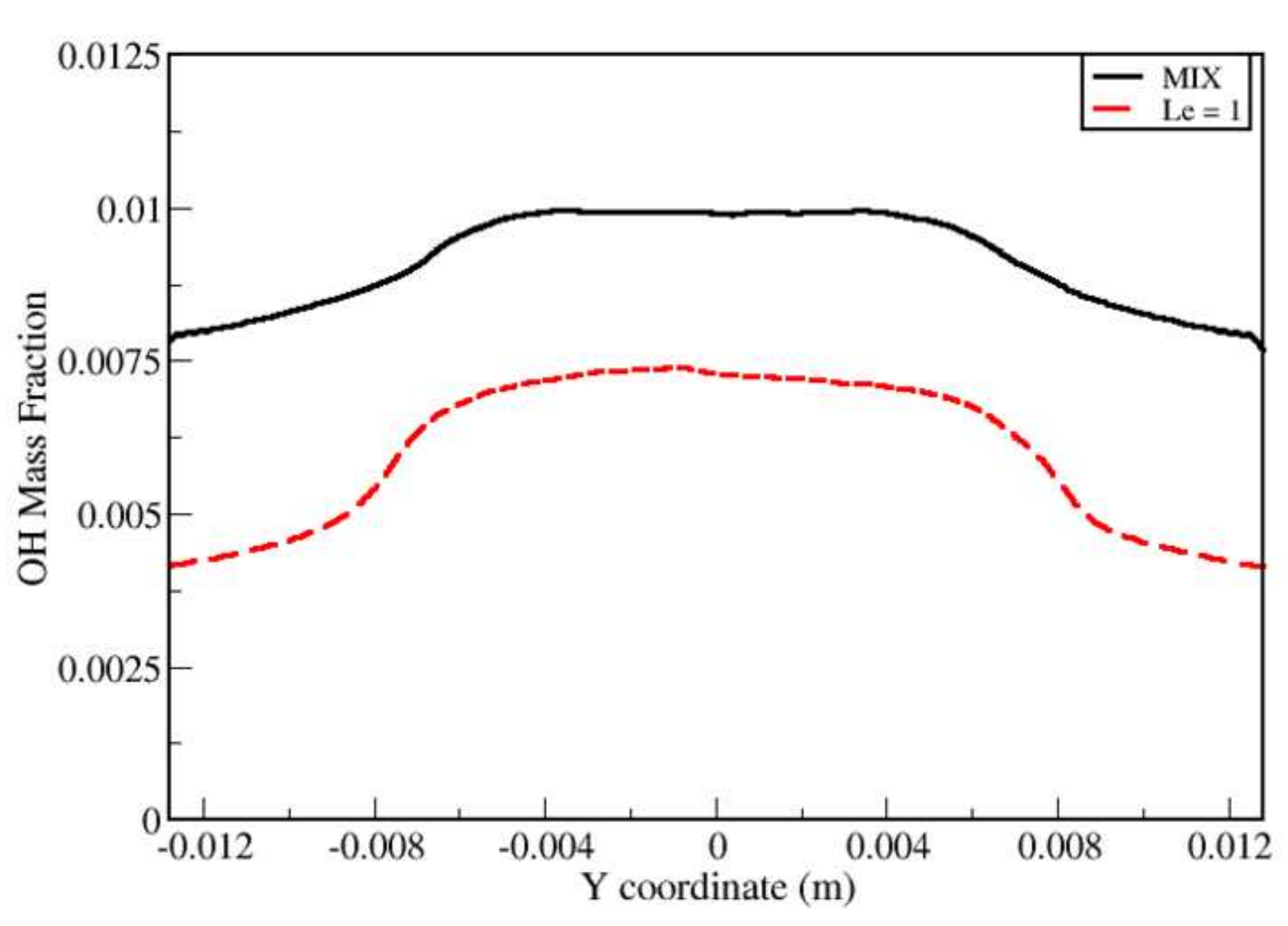




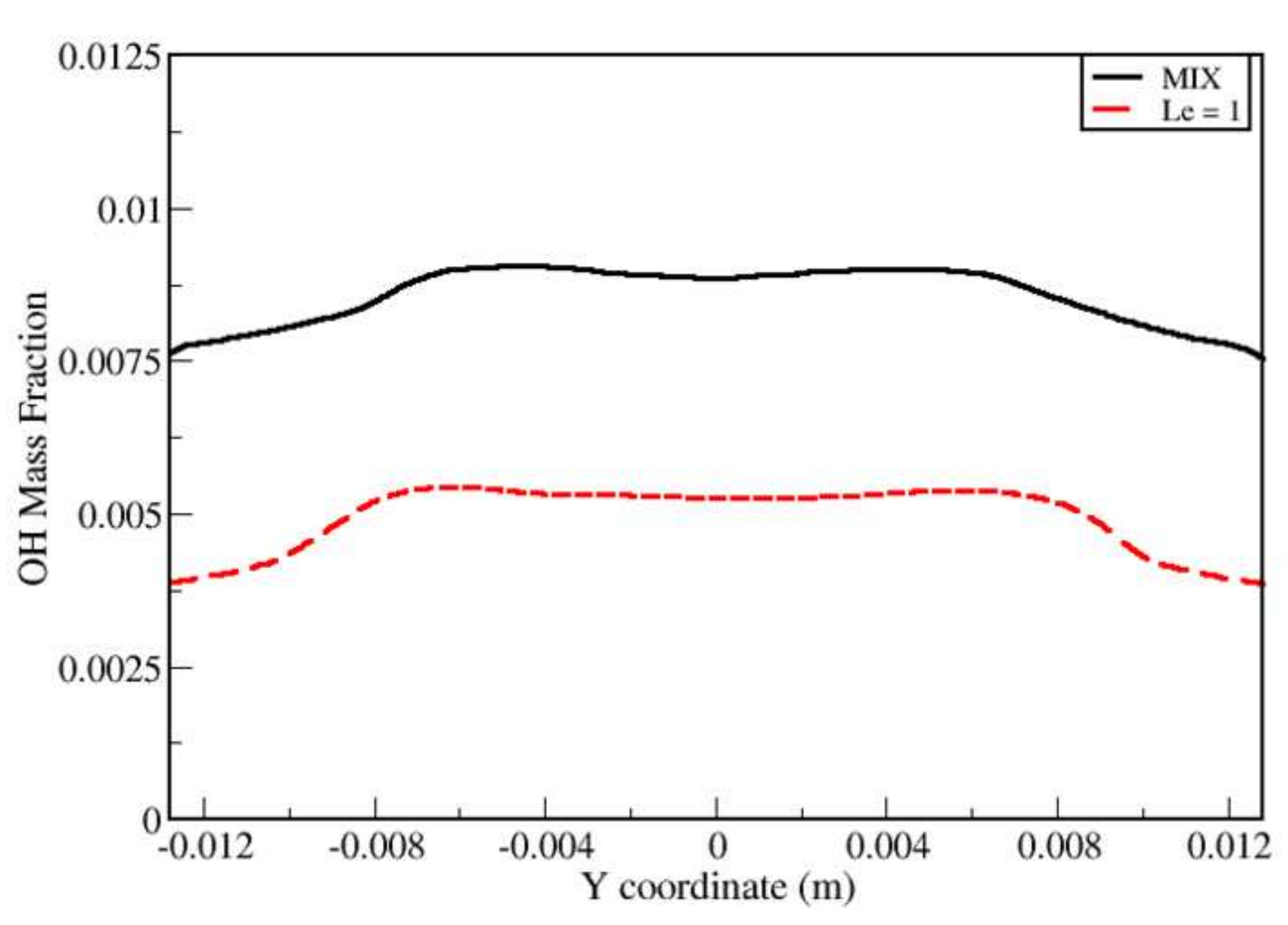




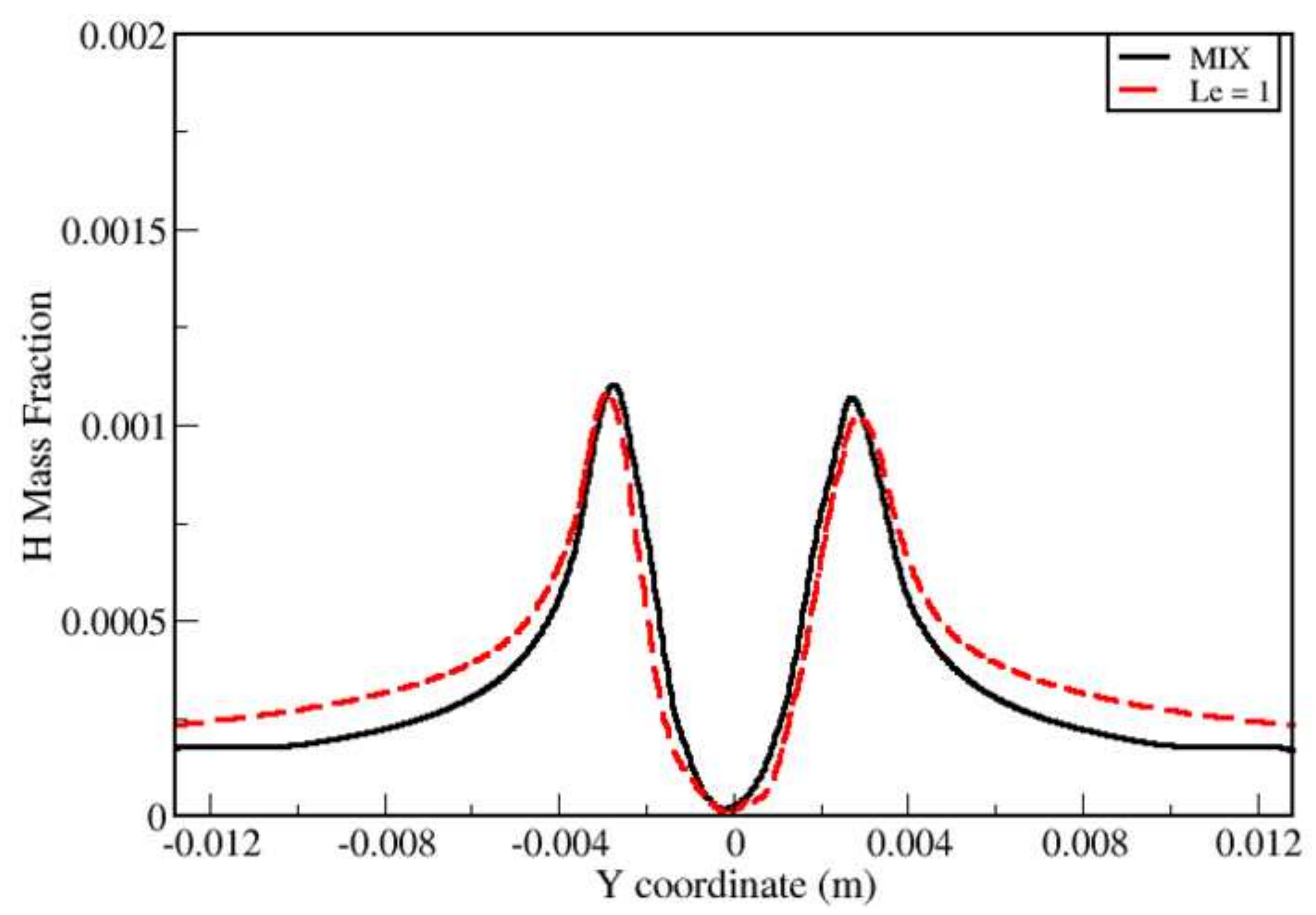




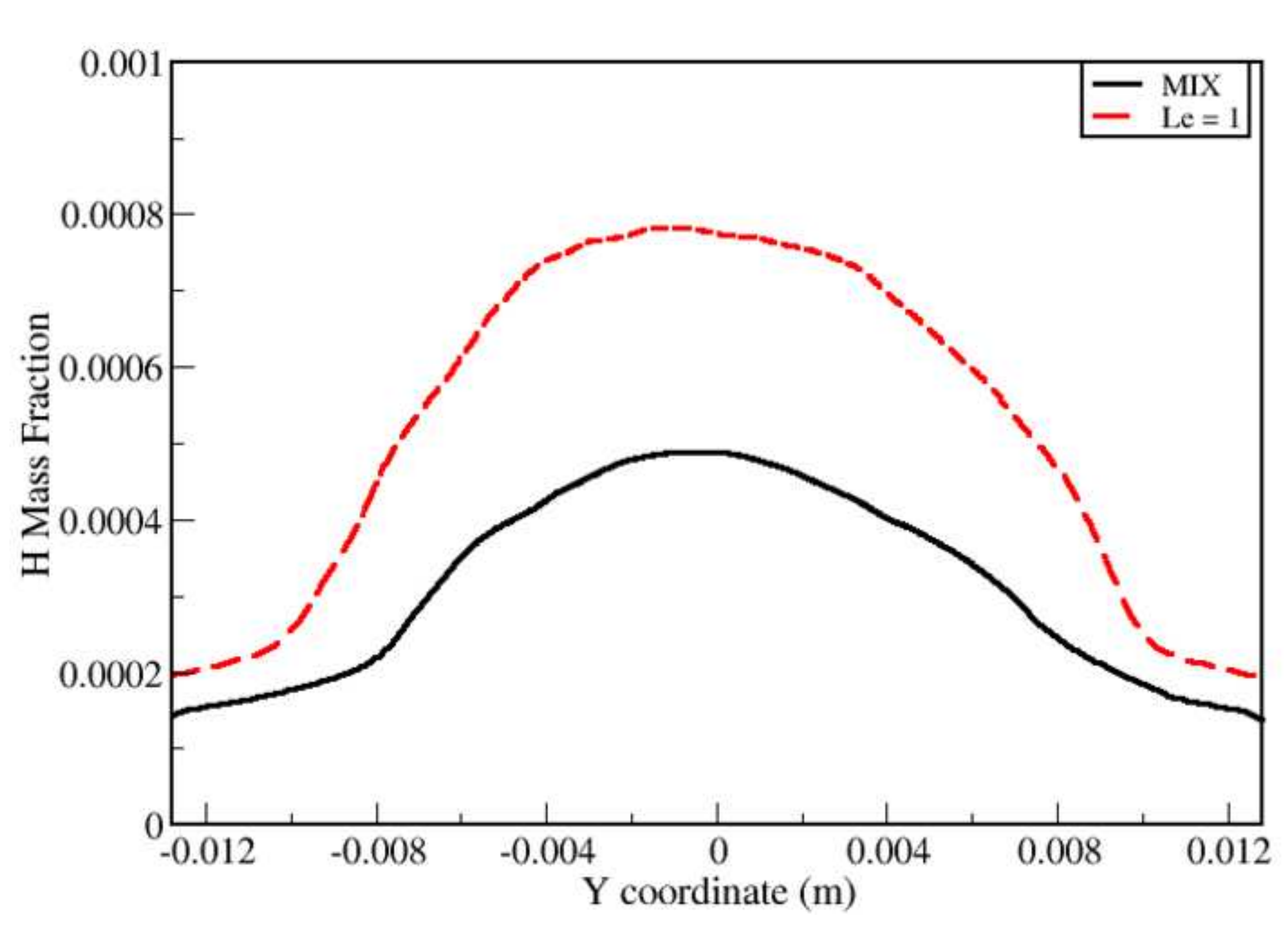




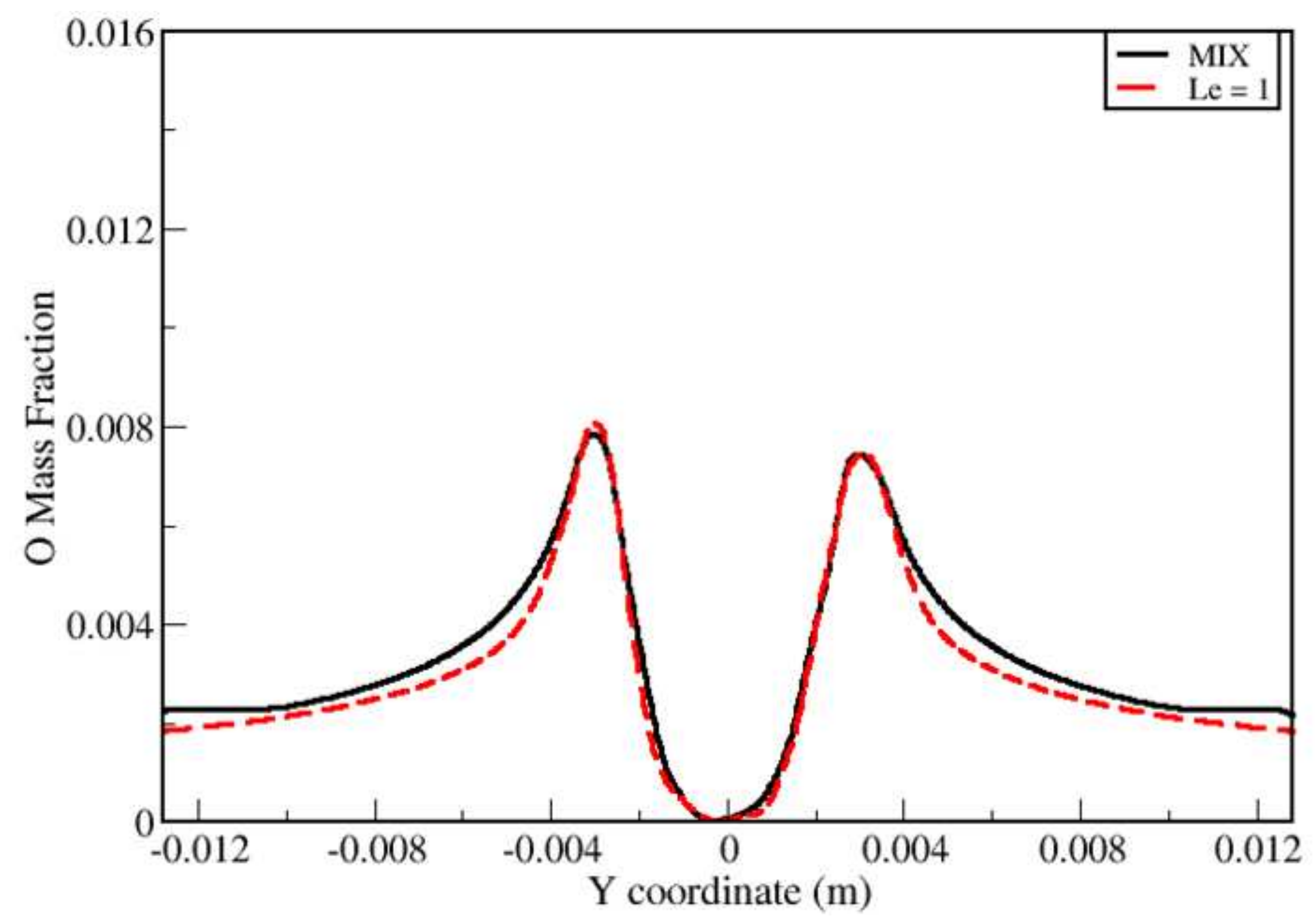




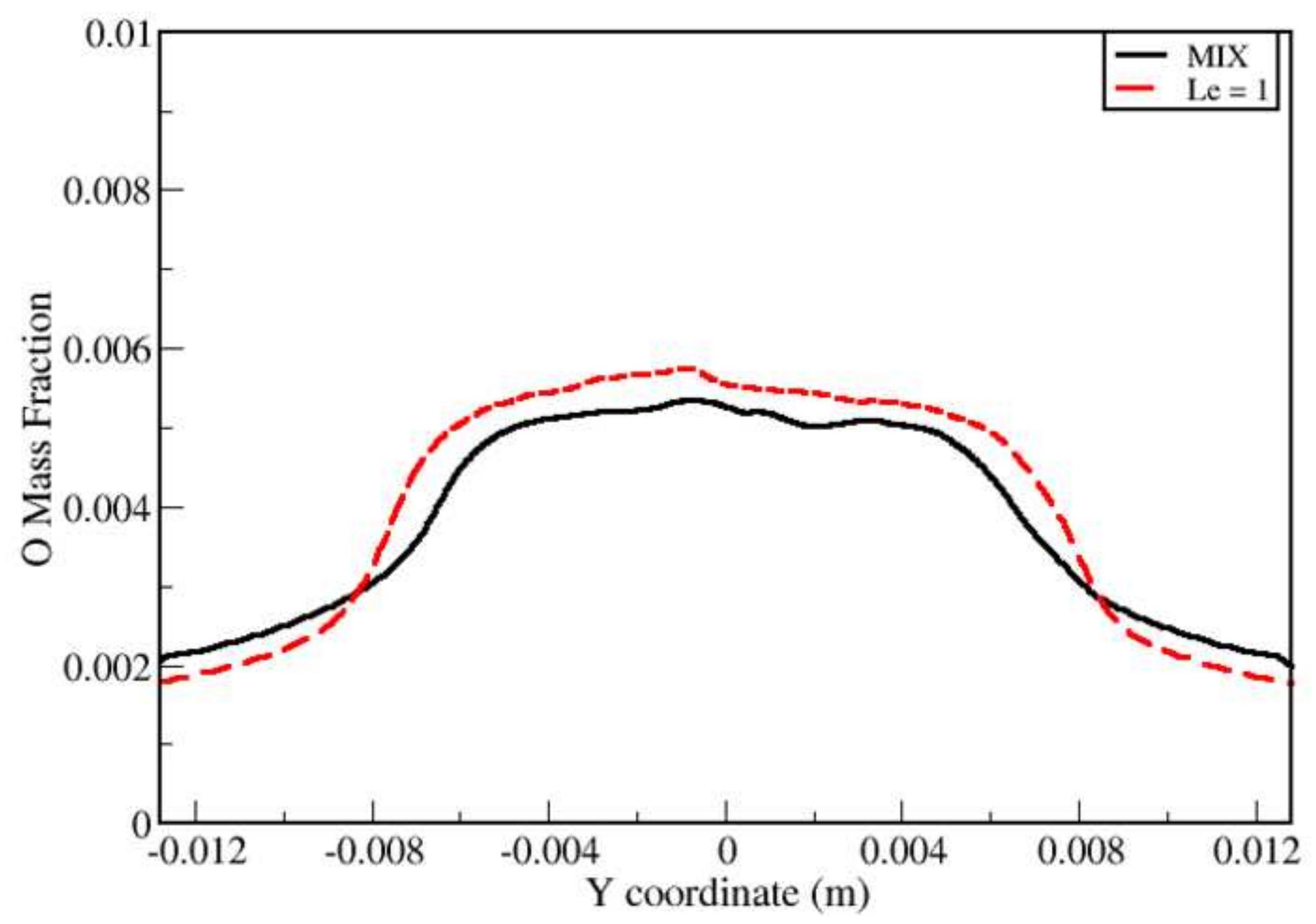




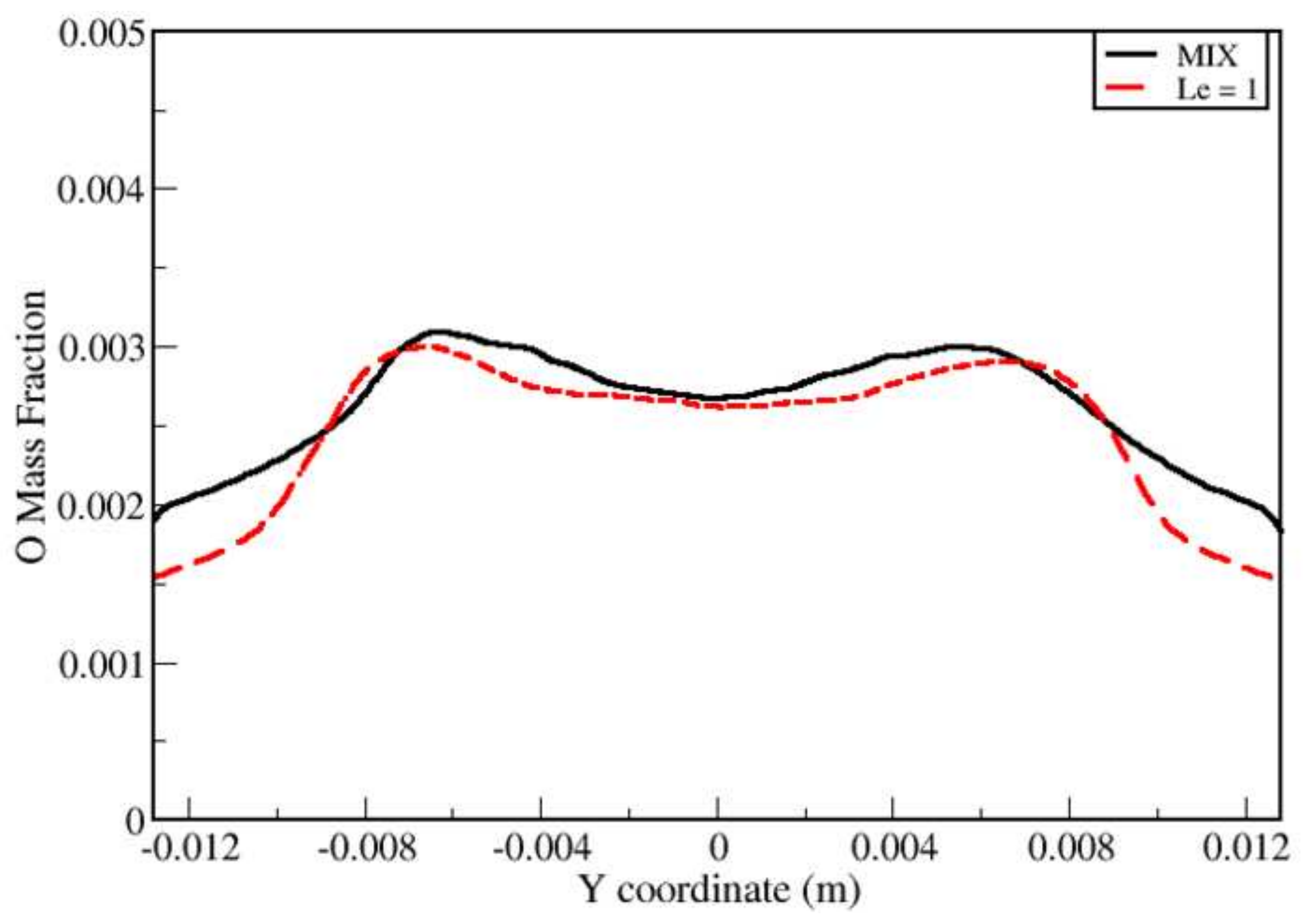




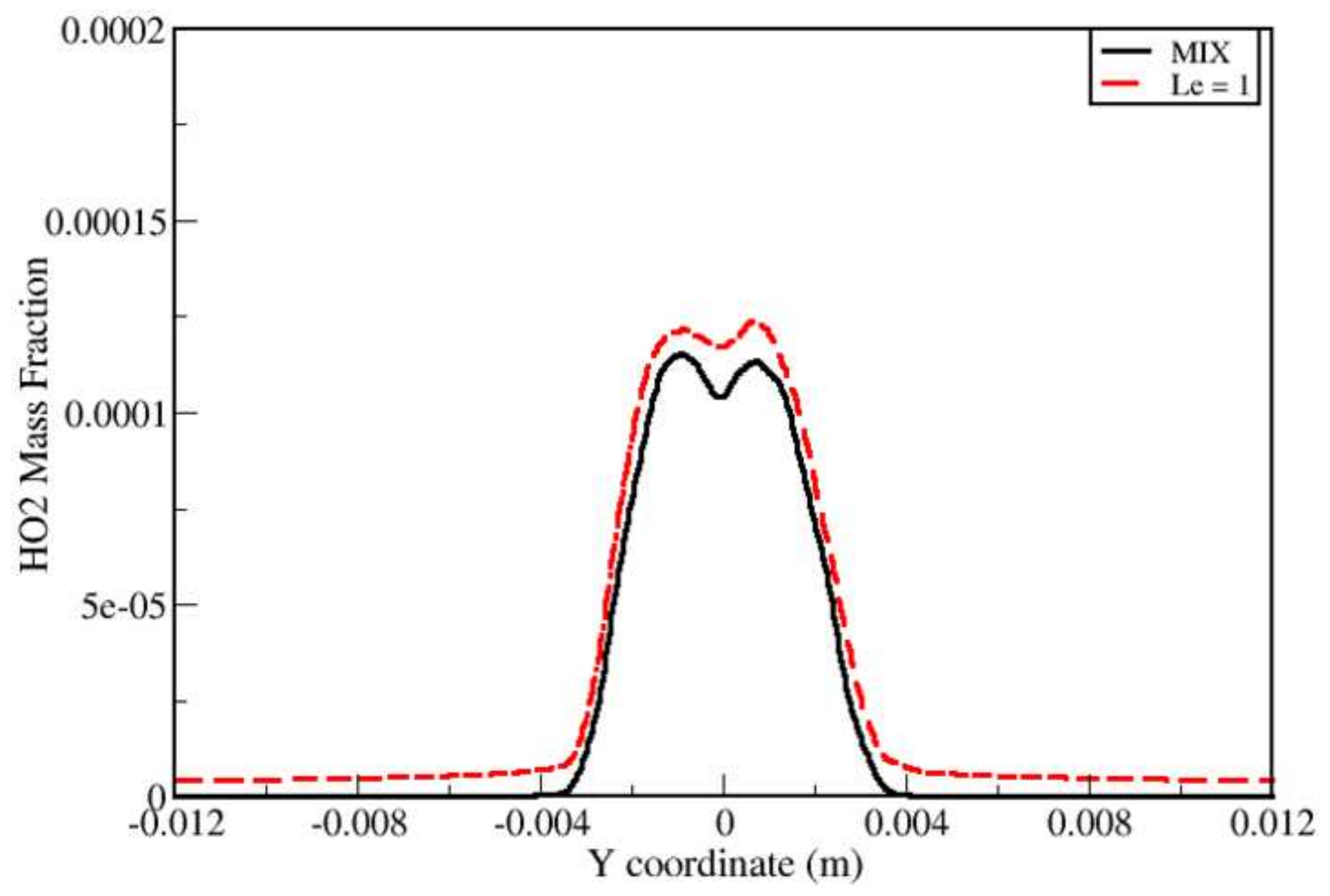




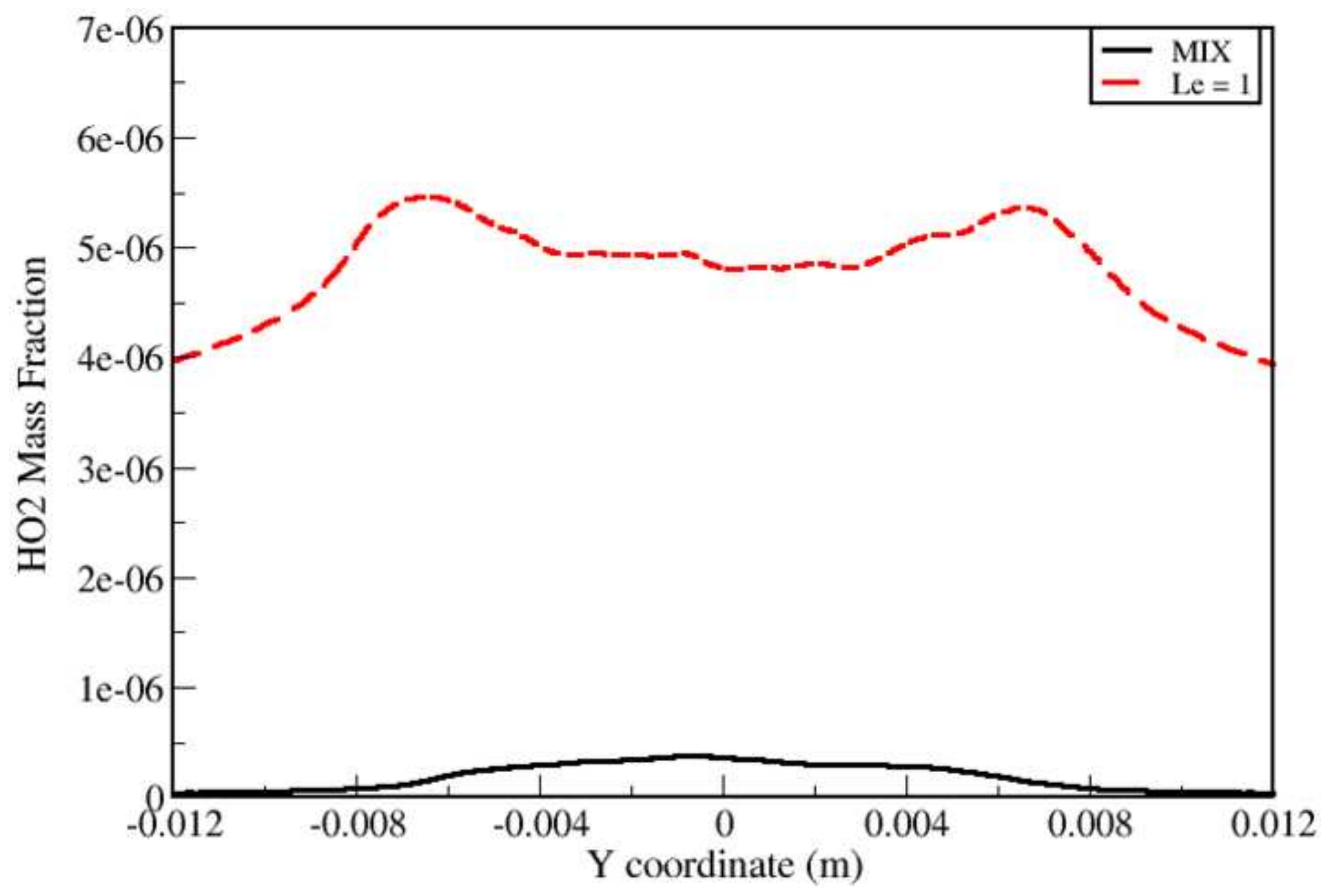




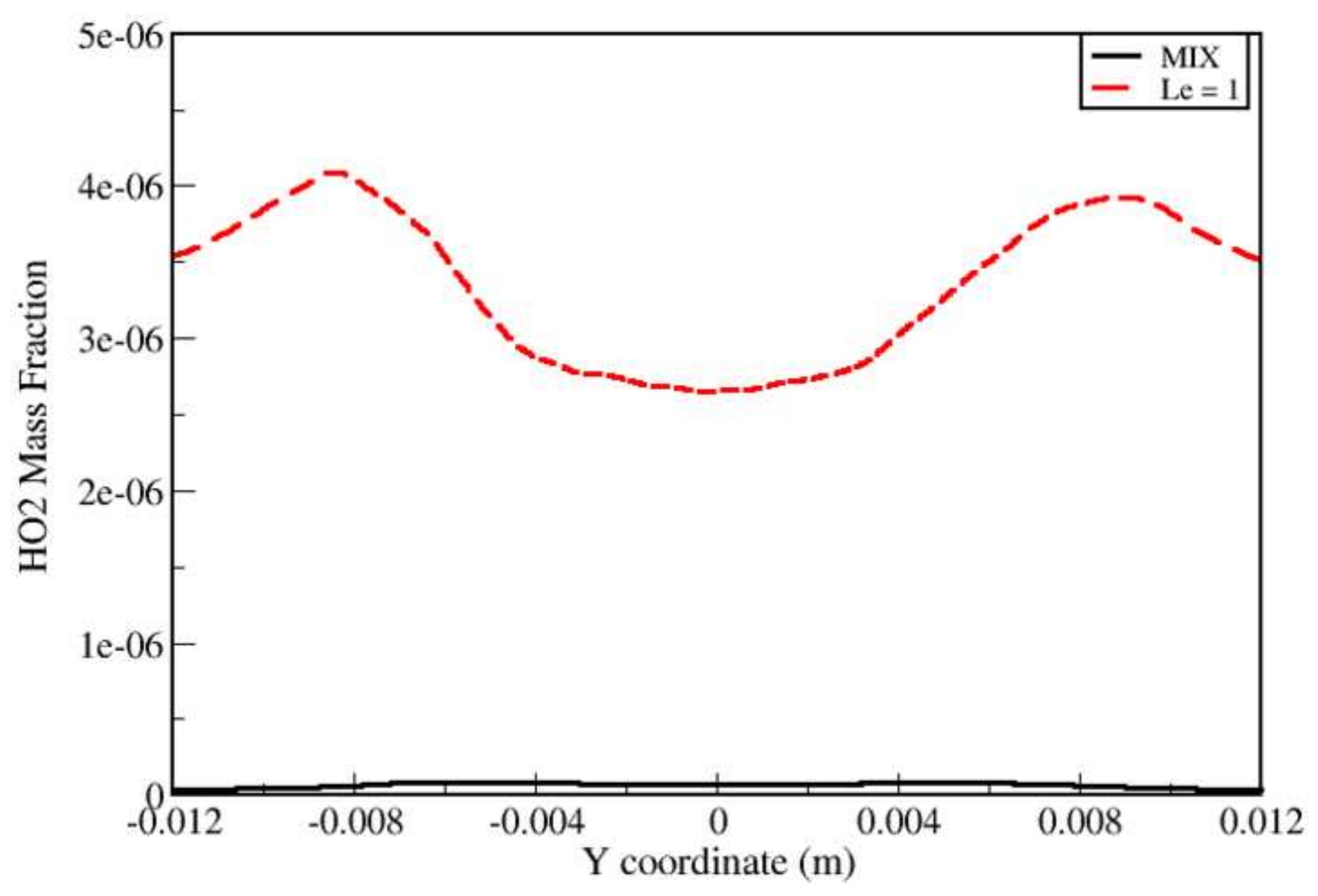




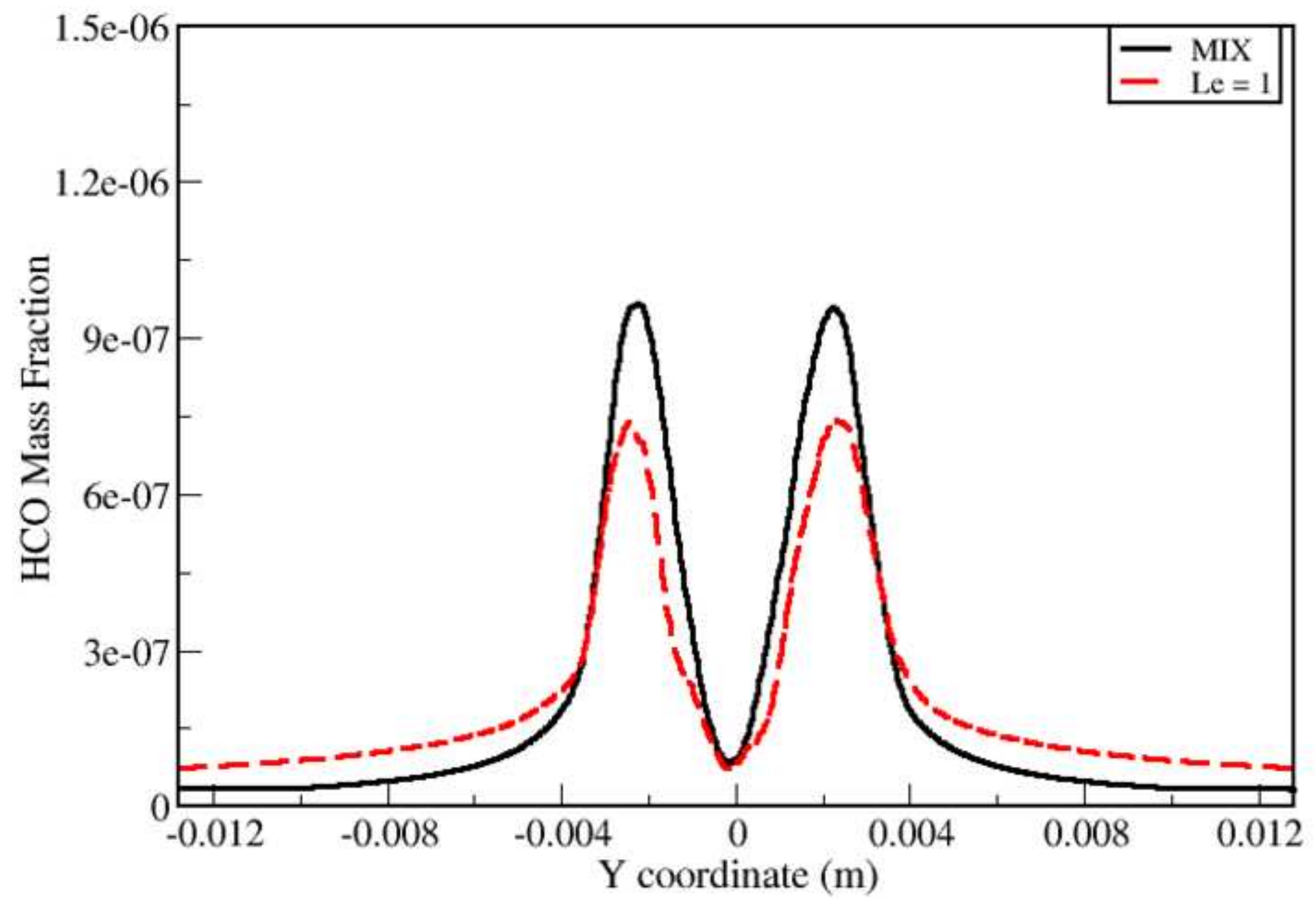




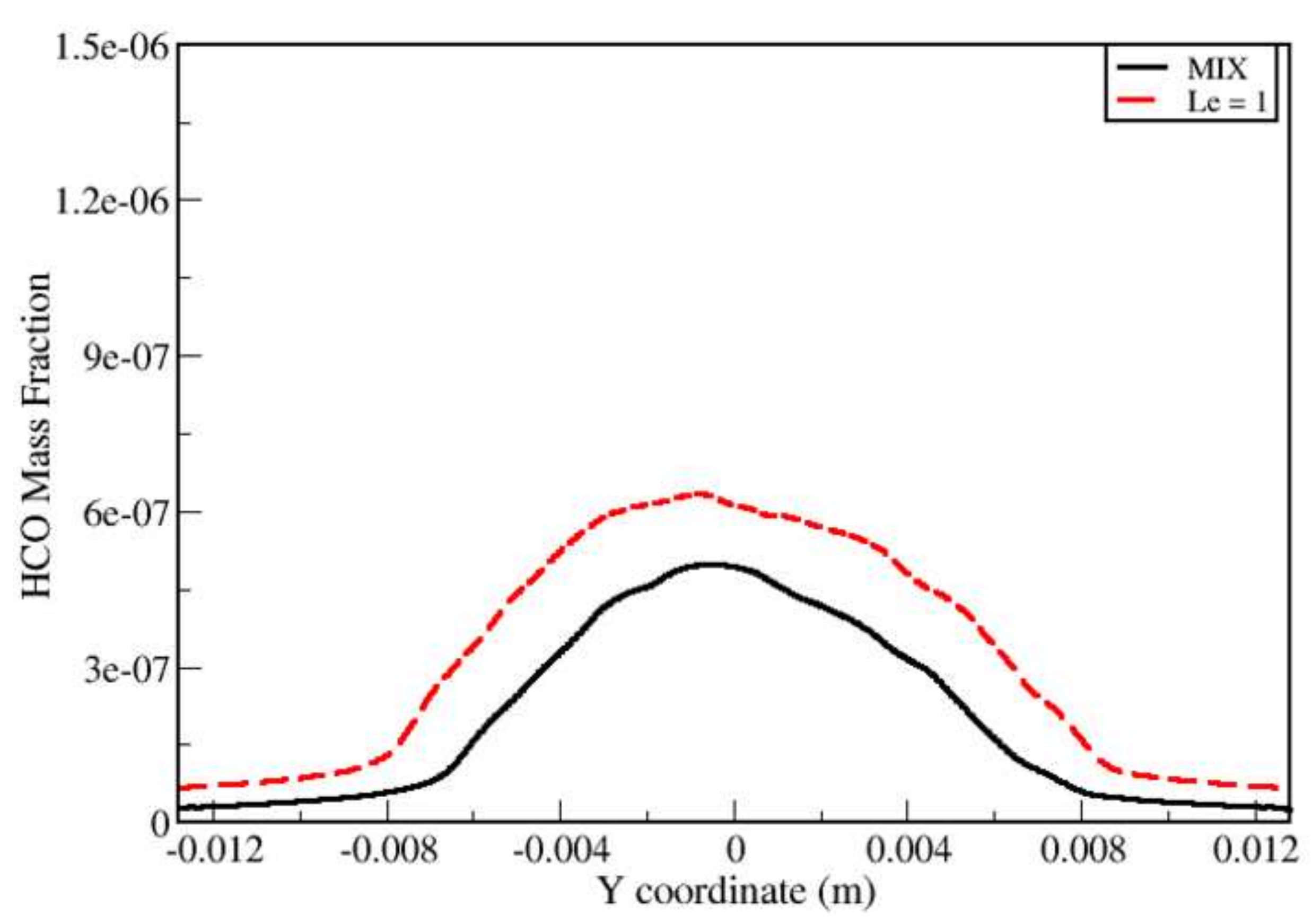




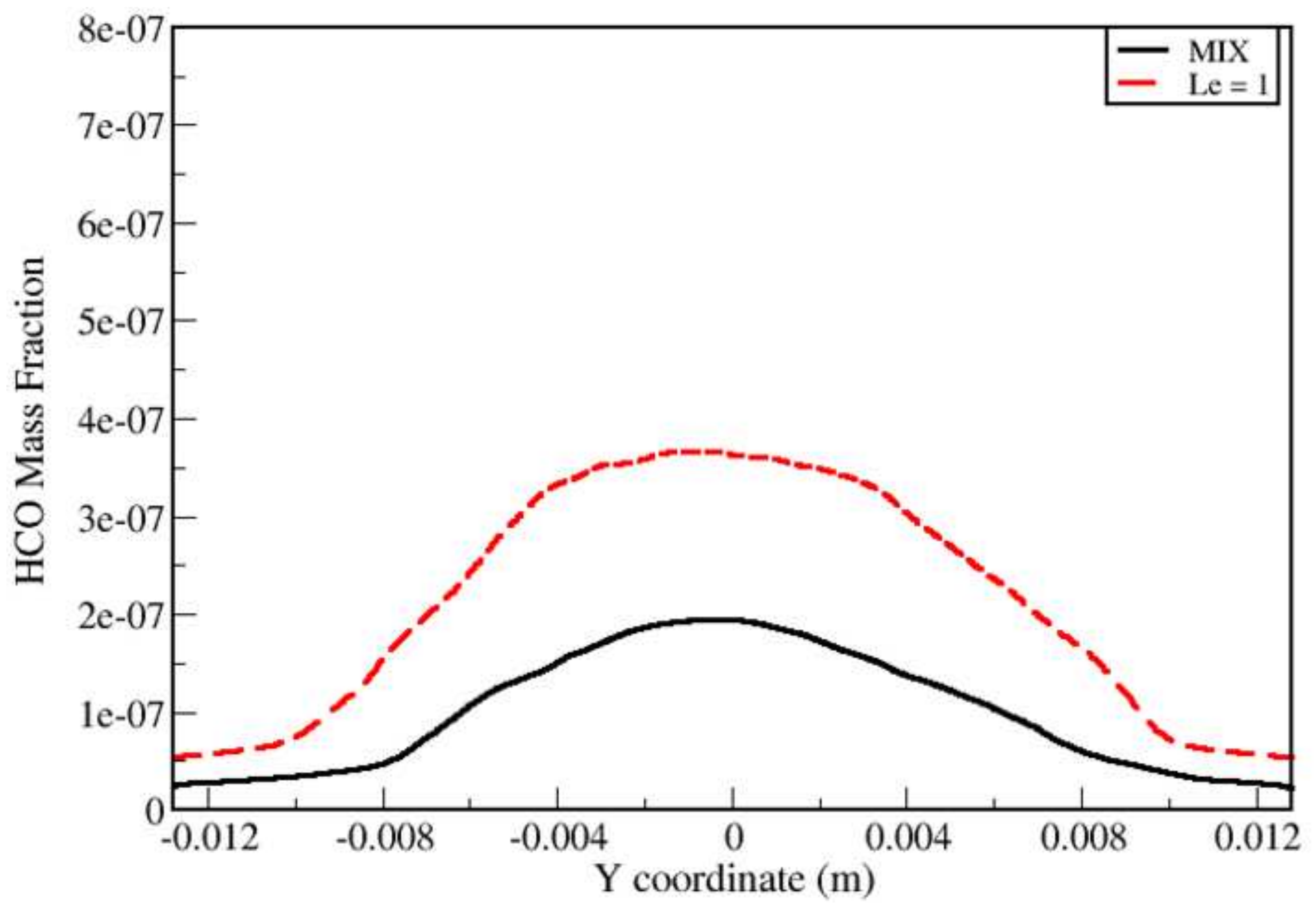




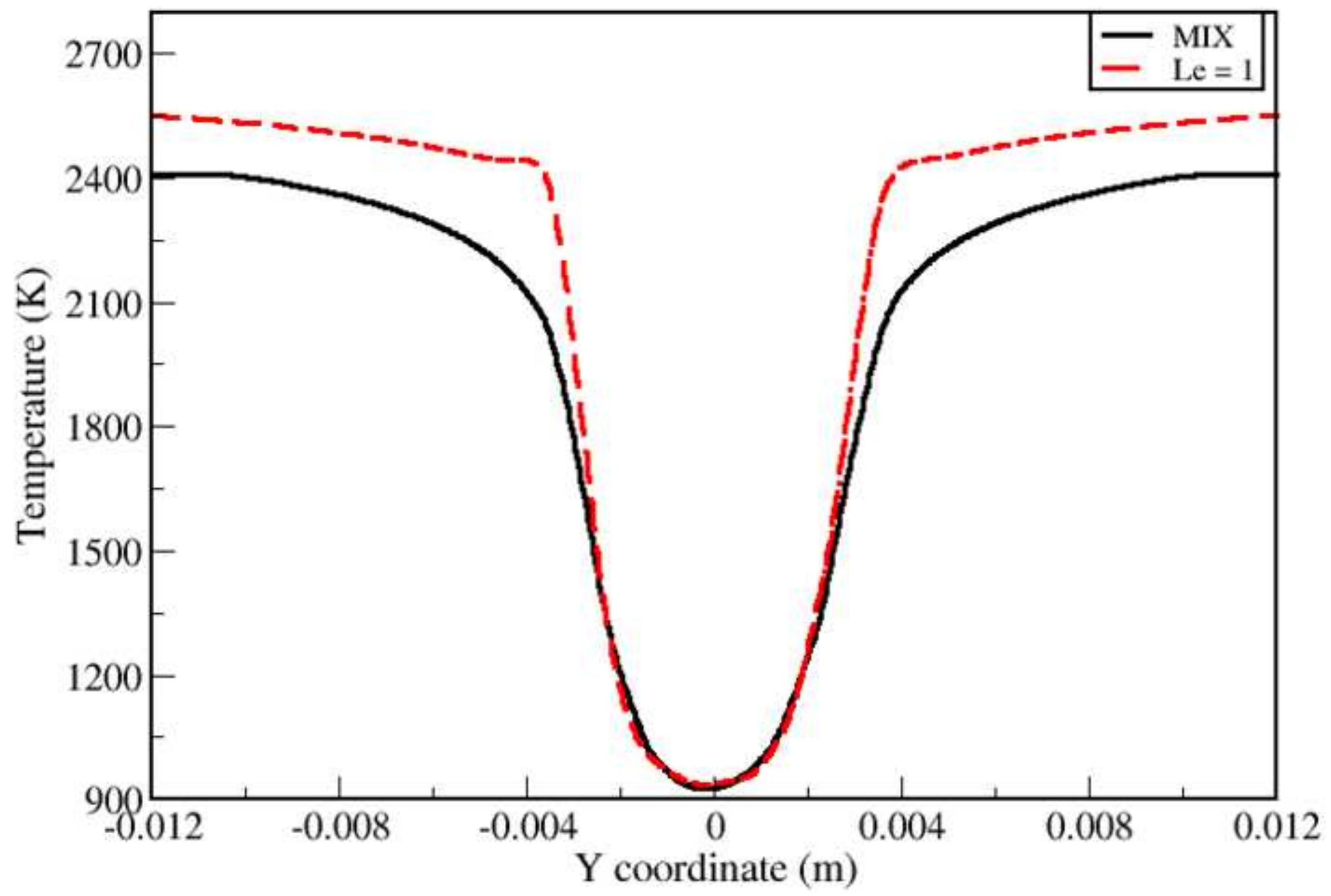




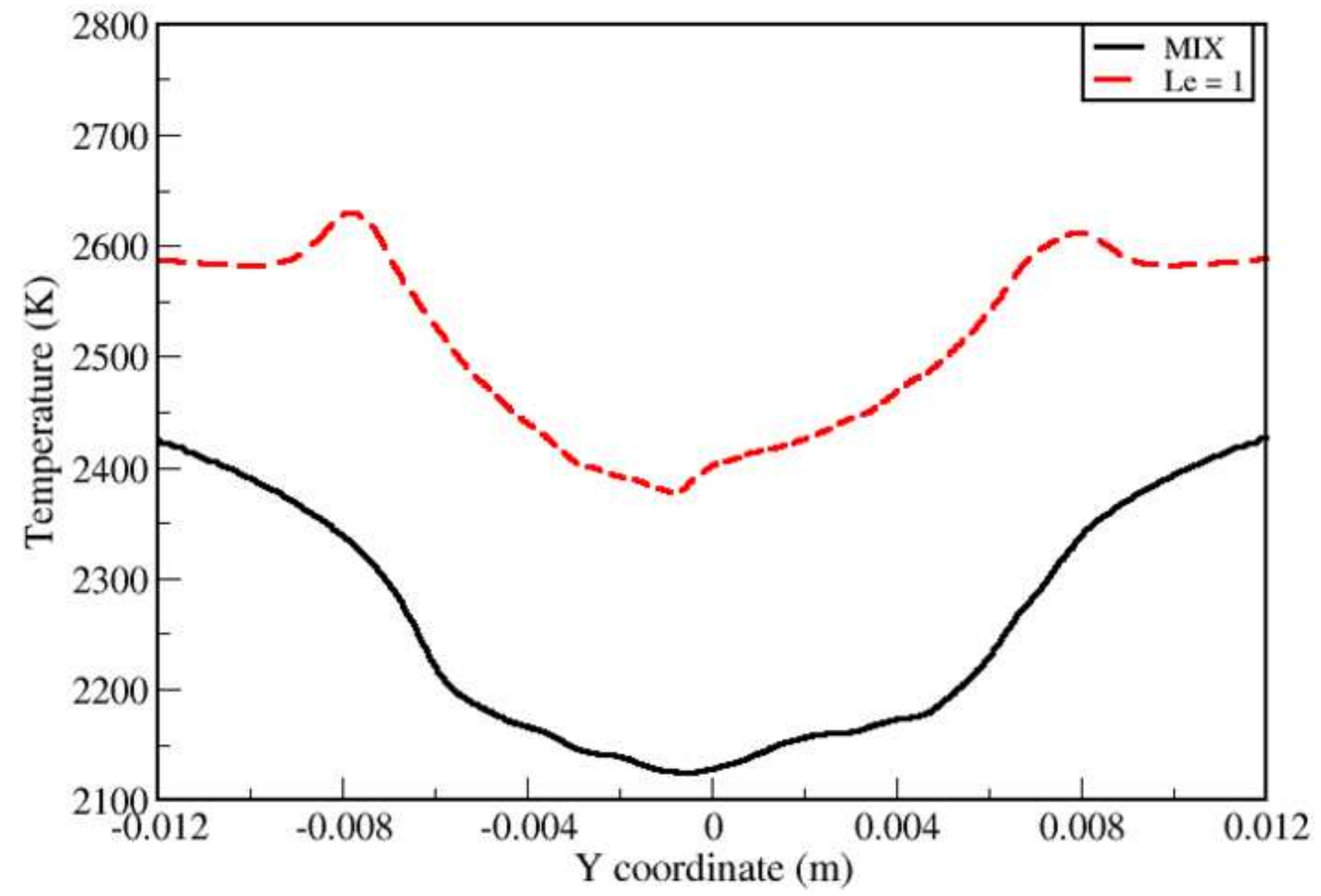




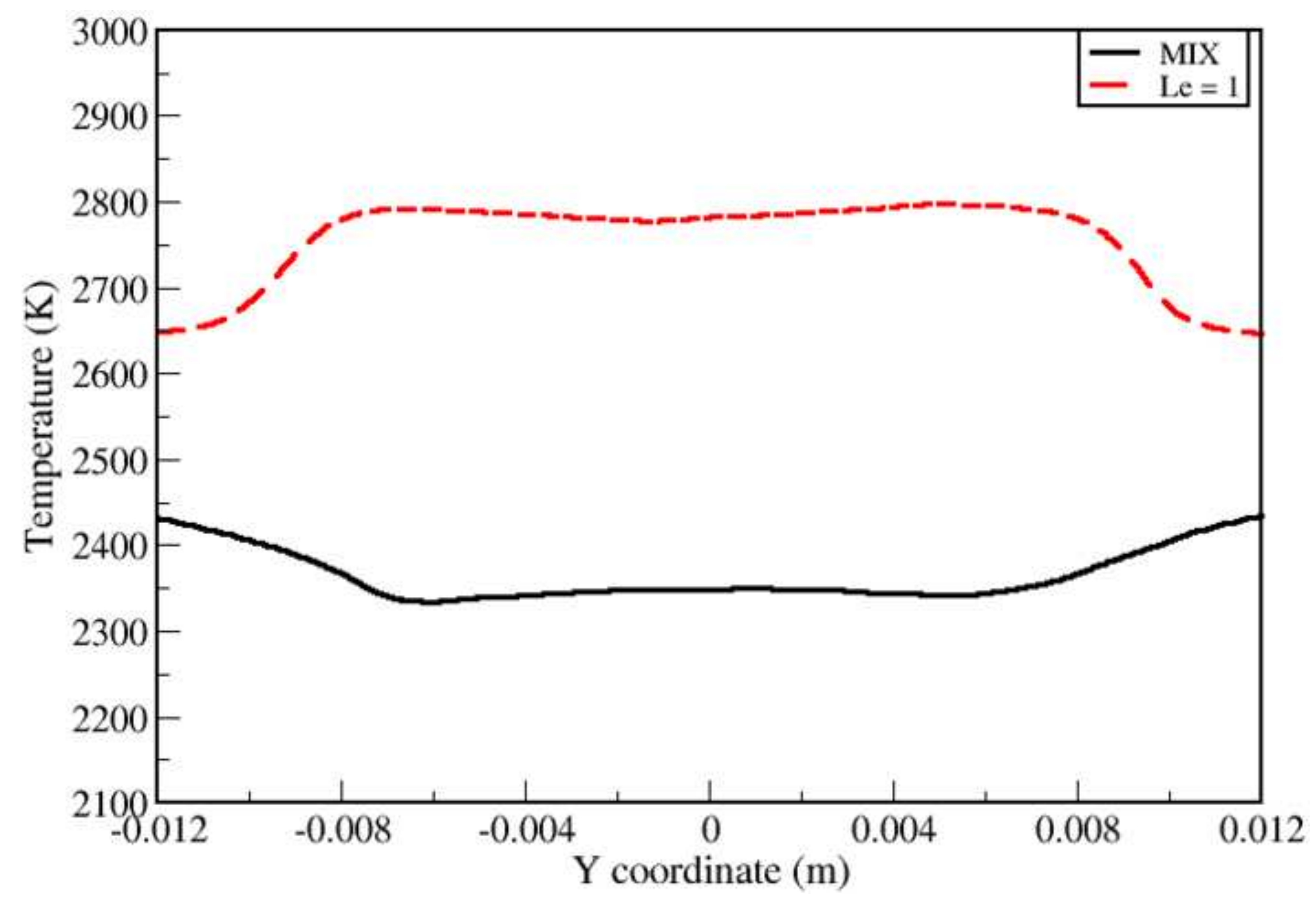




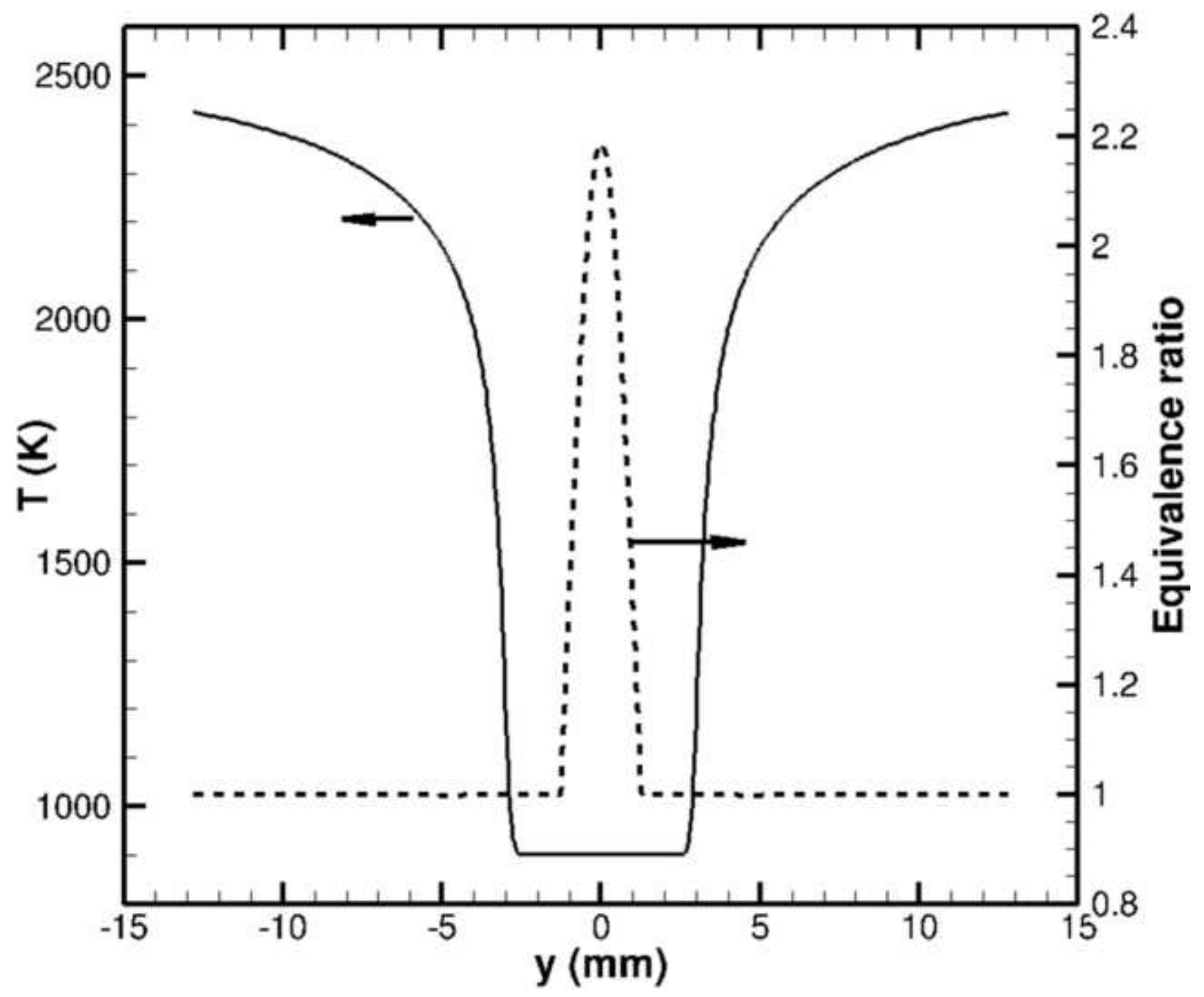




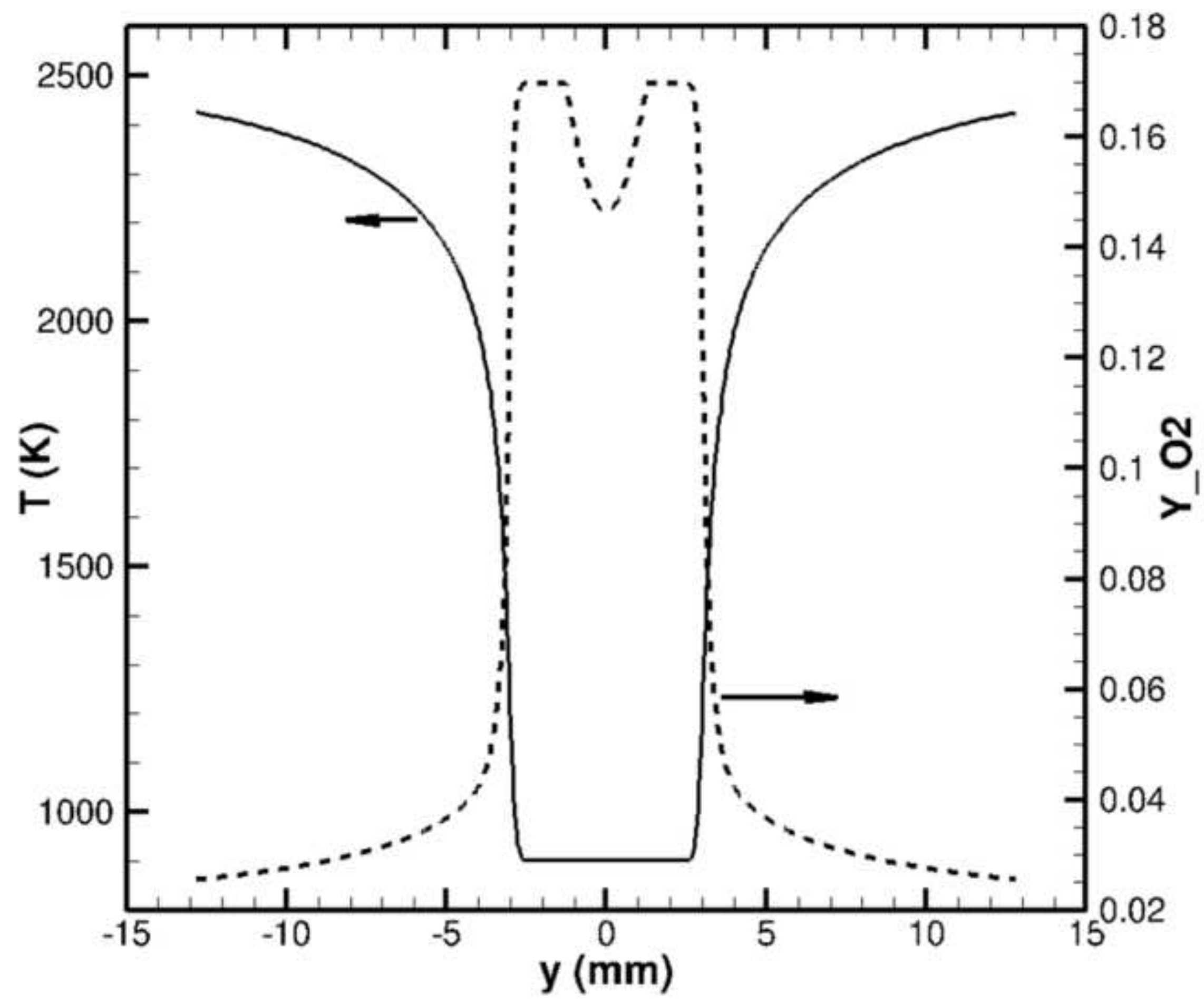

